\title{
Bisbiphenyl Phosphines. Structure and Synthesis of Gold(I) Alkene $\pi$-Complexes with Variable Phosphine Donicity and Enhanced Stability.
}

Carolin Griebel, Dwaine D. Hodges, Brock R. Yager, Fred L. Liu, Wentong Zhou, Katarina J. Makaravage, Yuyang Zhu, ${ }^{*}$ Skylar G. Norman, Ruichen Lan, Cynthia S. Day, Amanda C. Jones.

Department of Chemistry, Wake Forest University, Salem Hall, Box 7486, Winston-Salem, NC, 27109, USA.

\section{Contents}

I. NMR Spectra for compounds with Ligand 1, di([1,1'-biphenyl]-2-yl)(phenoxy)phosphane:... S2

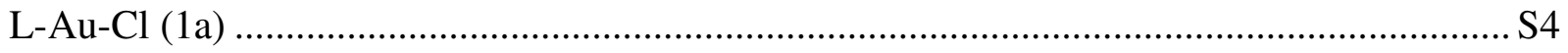

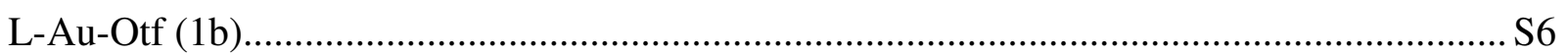

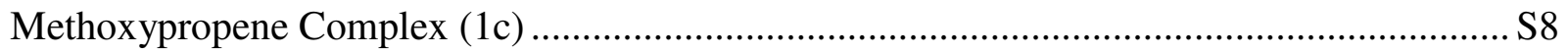

II. Experimental Details and NMR Spectra for compounds with di([1,1'-biphenyl]-2-

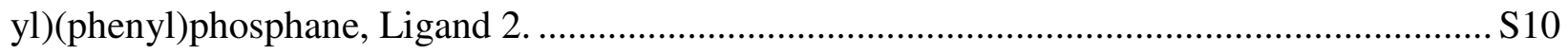

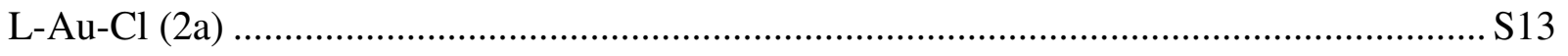

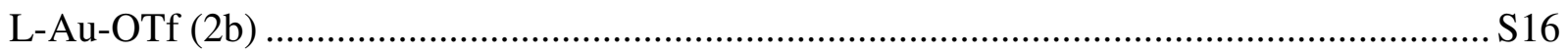

Methoxypropene Complex (2c) ..................................................................................... S19

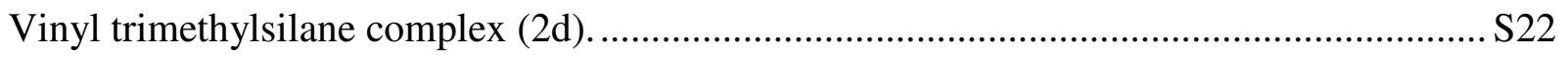

III. Chemistry of di([1,1'-biphenyl]-2-yl)(tert-butyl)phosphane, Ligand 3 ………….............. S25

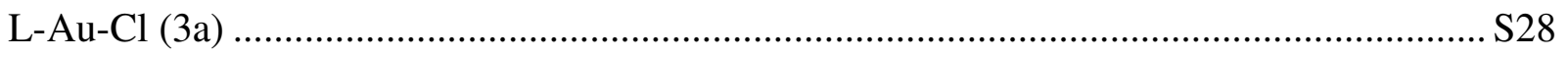

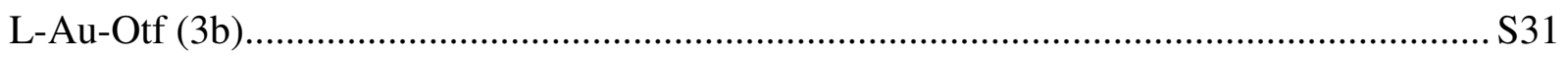

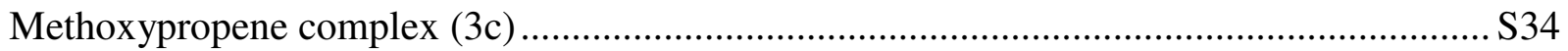

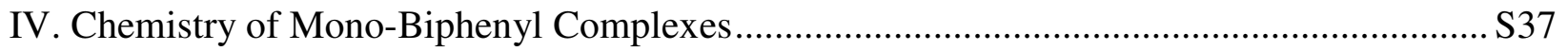

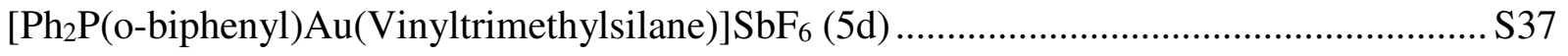

Appearance of [(Diphenylbiphenyl $\left.)_{2} \mathrm{Au}\right] \mathrm{SbF}_{6}$ Decomposition Product and Confirmation of ${ }^{31} \mathrm{p}$

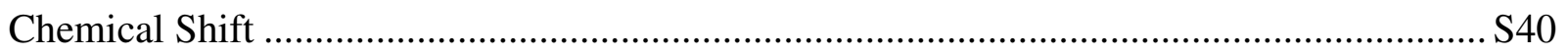

t-Bu $2 \mathrm{P}(\mathrm{o}$-biphenyl)]Au(Vinyltrimethylsilane)SbF6 (6d).................................................... S42

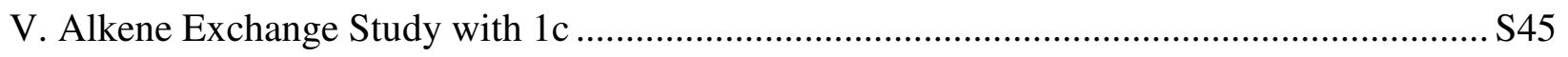

VI. ${ }^{1} \mathrm{H}$ Spectra showing Methoxypropene Hydrolysis byproducts........................................... $\mathrm{S} 48$ 
I. NMR Spectra for compounds with Ligand 1, di([1,1'-biphenyl]-2yl)(phenoxy)phosphane:

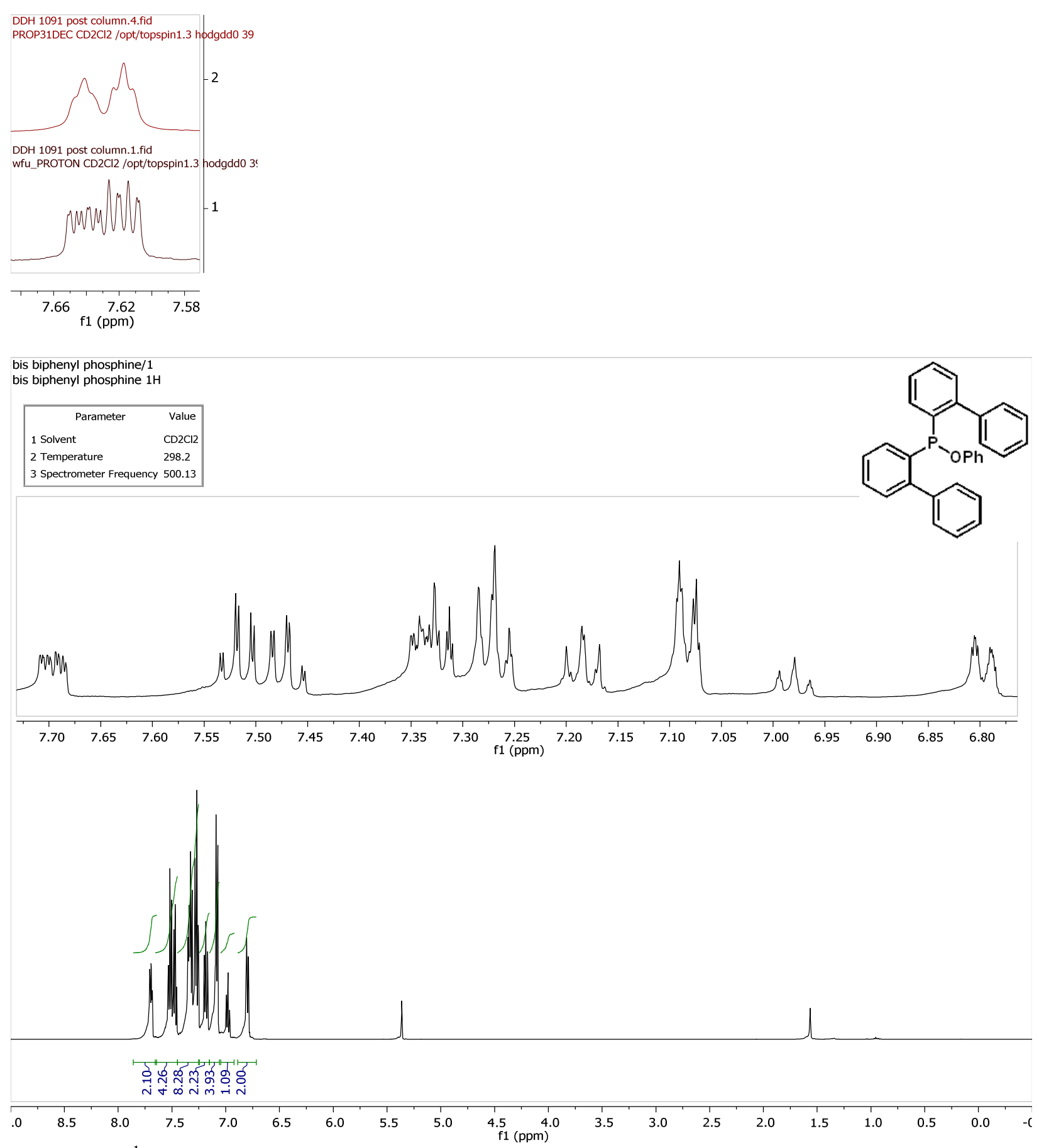

Figure S1. ${ }^{1} \mathrm{H}$ NMR spectrum of $1\left(500 \mathrm{MHz}, \mathrm{CD}_{2} \mathrm{Cl}_{2}\right)$ with above inset showing phosphorous decoupling. 


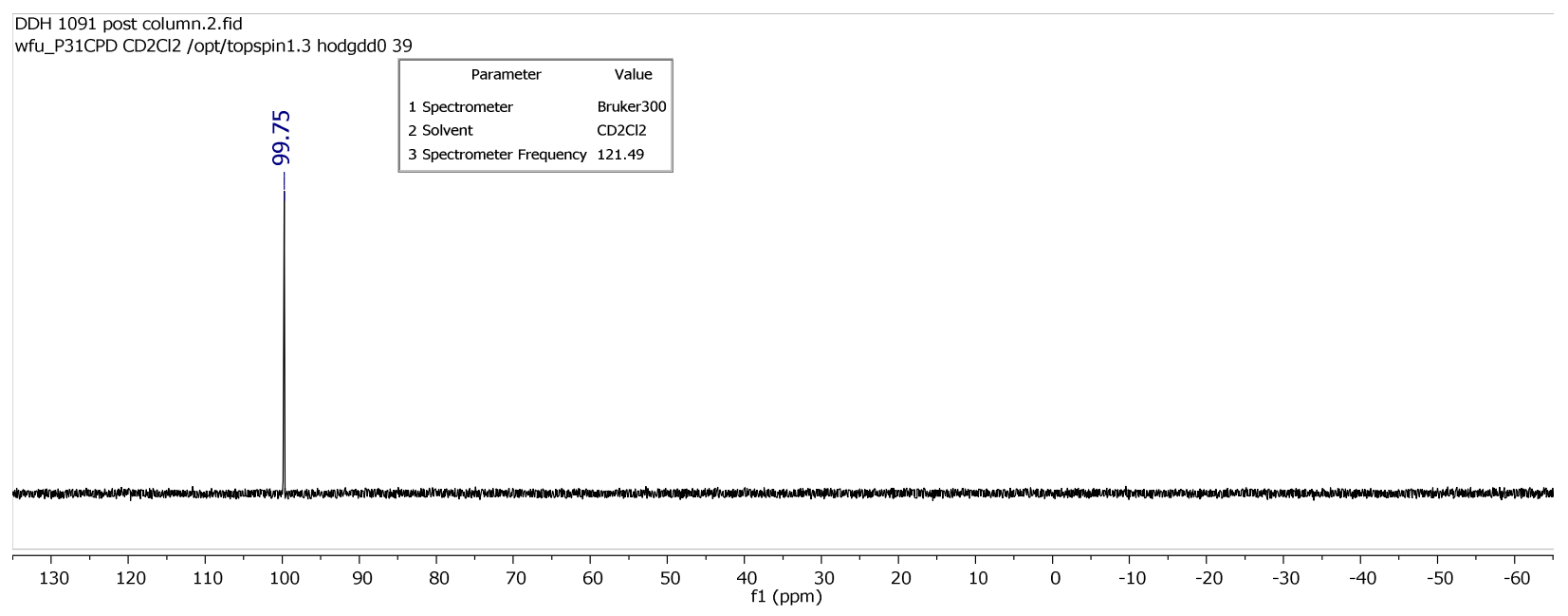

Figure S2. ${ }^{31} \mathrm{P}$ NMR spectrum of $\mathbf{1}\left(121.5 \mathrm{MHz}, \mathrm{CD}_{2} \mathrm{Cl}_{2}\right)$.

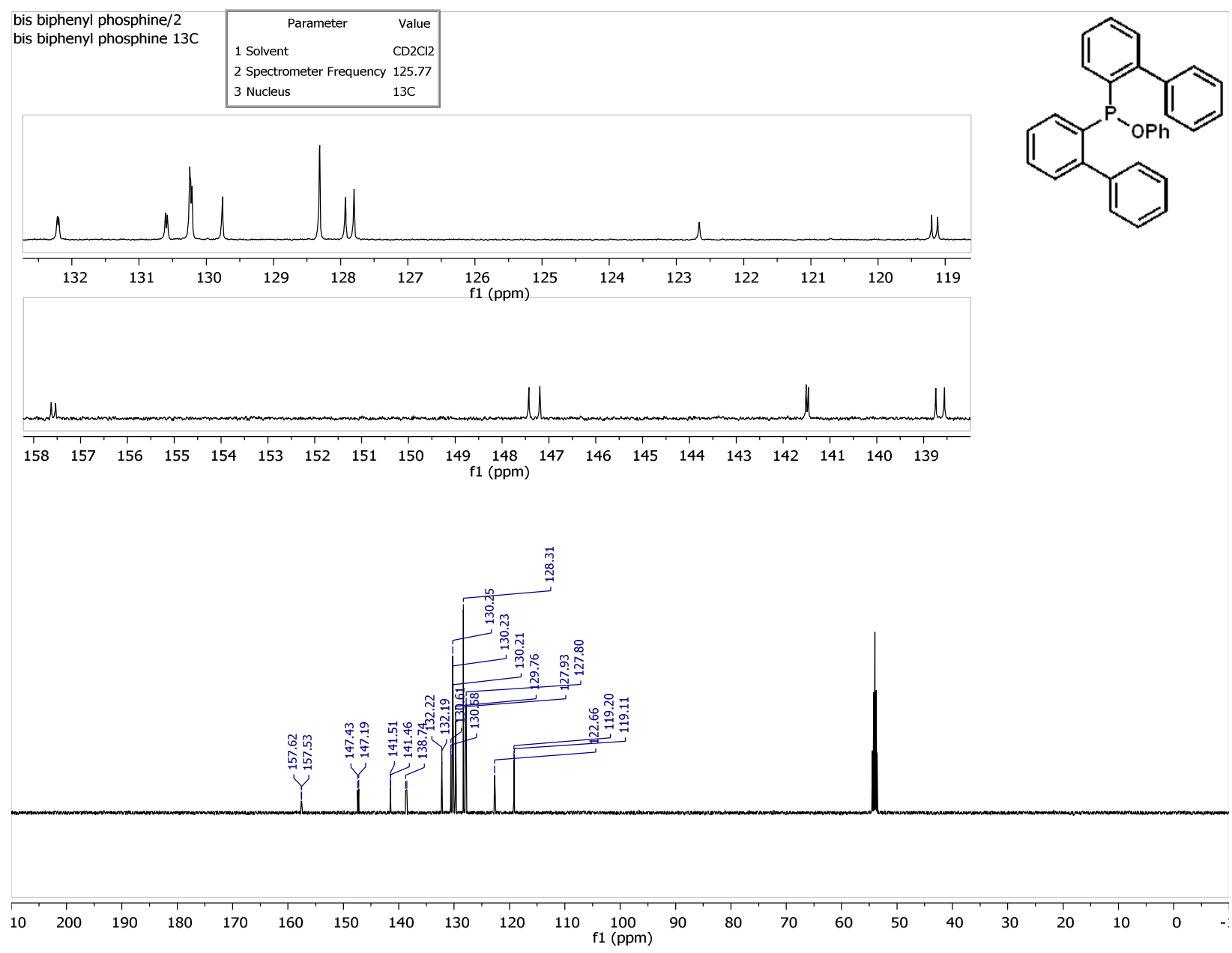

Figure S3. ${ }^{13} \mathrm{C}$ NMR spectrum of $1\left(125.77 \mathrm{MHz}, \mathrm{CD}_{2} \mathrm{Cl}_{2}\right)$.

S-3 


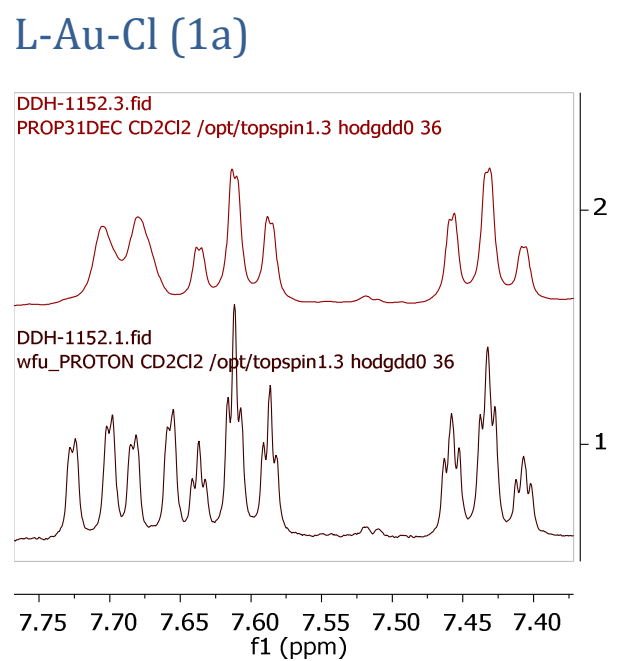

DDH-1152.1.fid

wfu_PROTON CD2CI2 /opt/topspin1.3 hodgdd0 36

\begin{tabular}{|lc|}
\hline \multicolumn{1}{|c|}{ Parameter } & Value \\
1 Solvent & $\mathrm{CD} 2 \mathrm{Cl} 2$ \\
2 Spectrometer Frequency & 300.13 \\
3 Nucleus & $1 \mathrm{H}$ \\
\hline
\end{tabular}
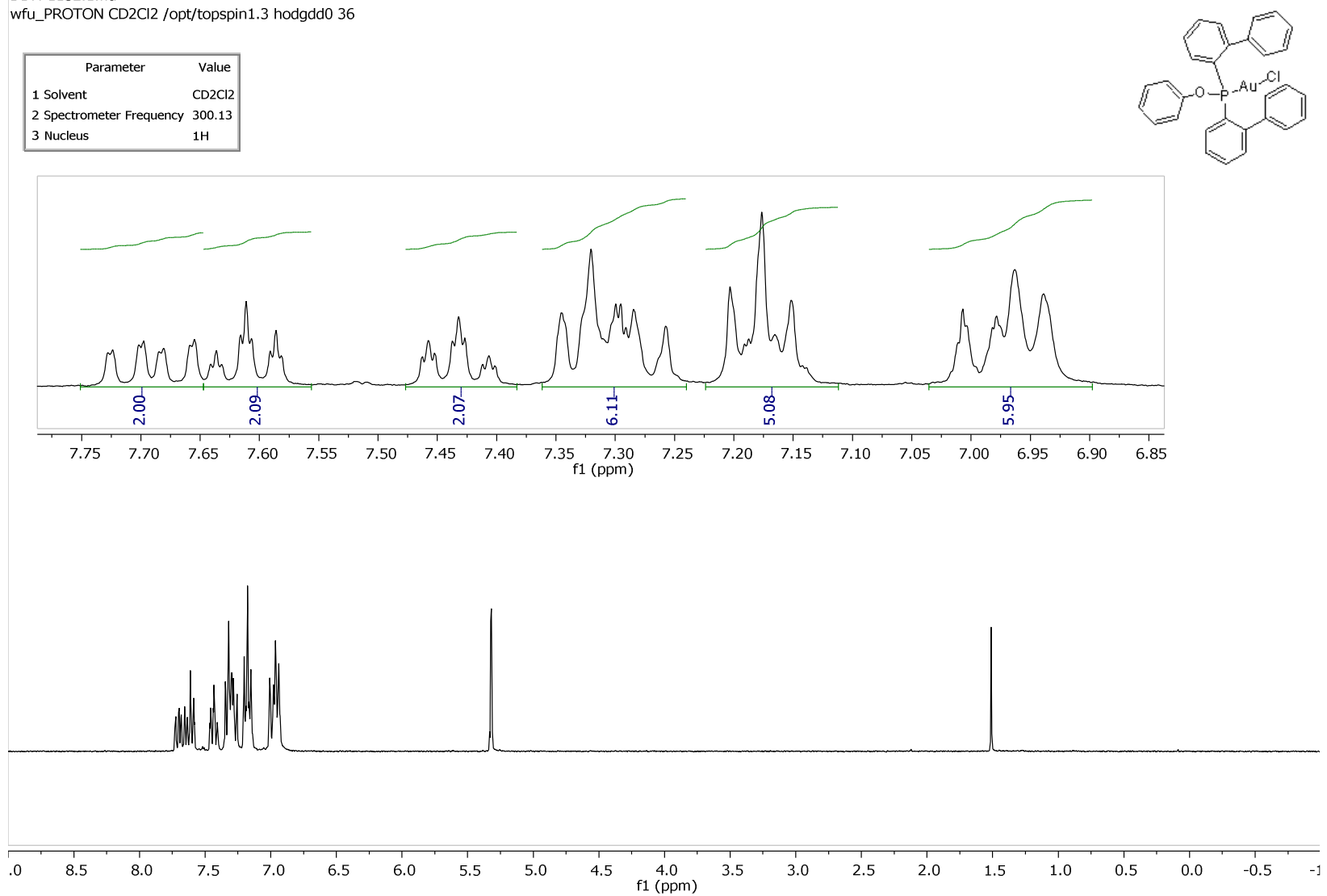

Figure S4. ${ }^{1} \mathrm{H}$ NMR spectrum of $\mathbf{1 a}\left(300 \mathrm{MHz}, \mathrm{CD}_{2} \mathrm{Cl}_{2}\right)$ with above inset showing phosphorous decoupling. 


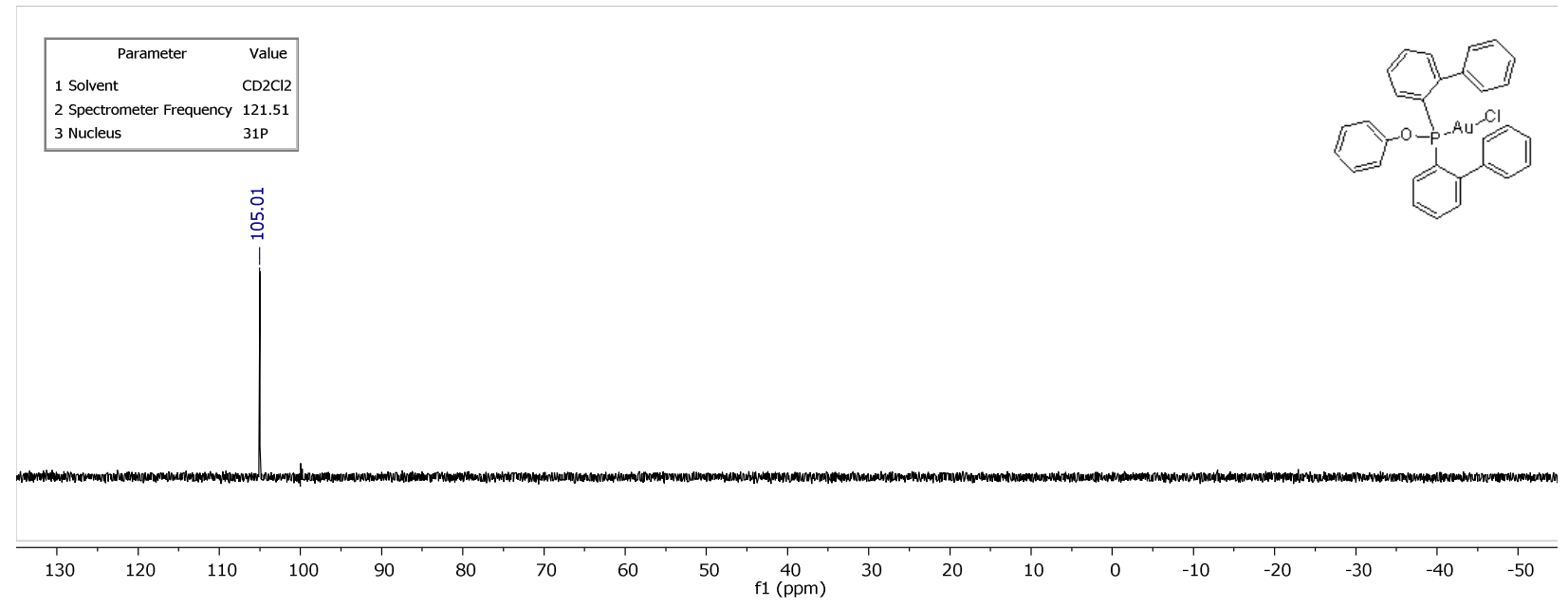

Figure S5. ${ }^{31} \mathrm{P}$ NMR spectrum of $\mathbf{1 a}\left(121.51 \mathrm{Mhz}, \mathrm{CD}_{2} \mathrm{Cl}_{2}\right)$.

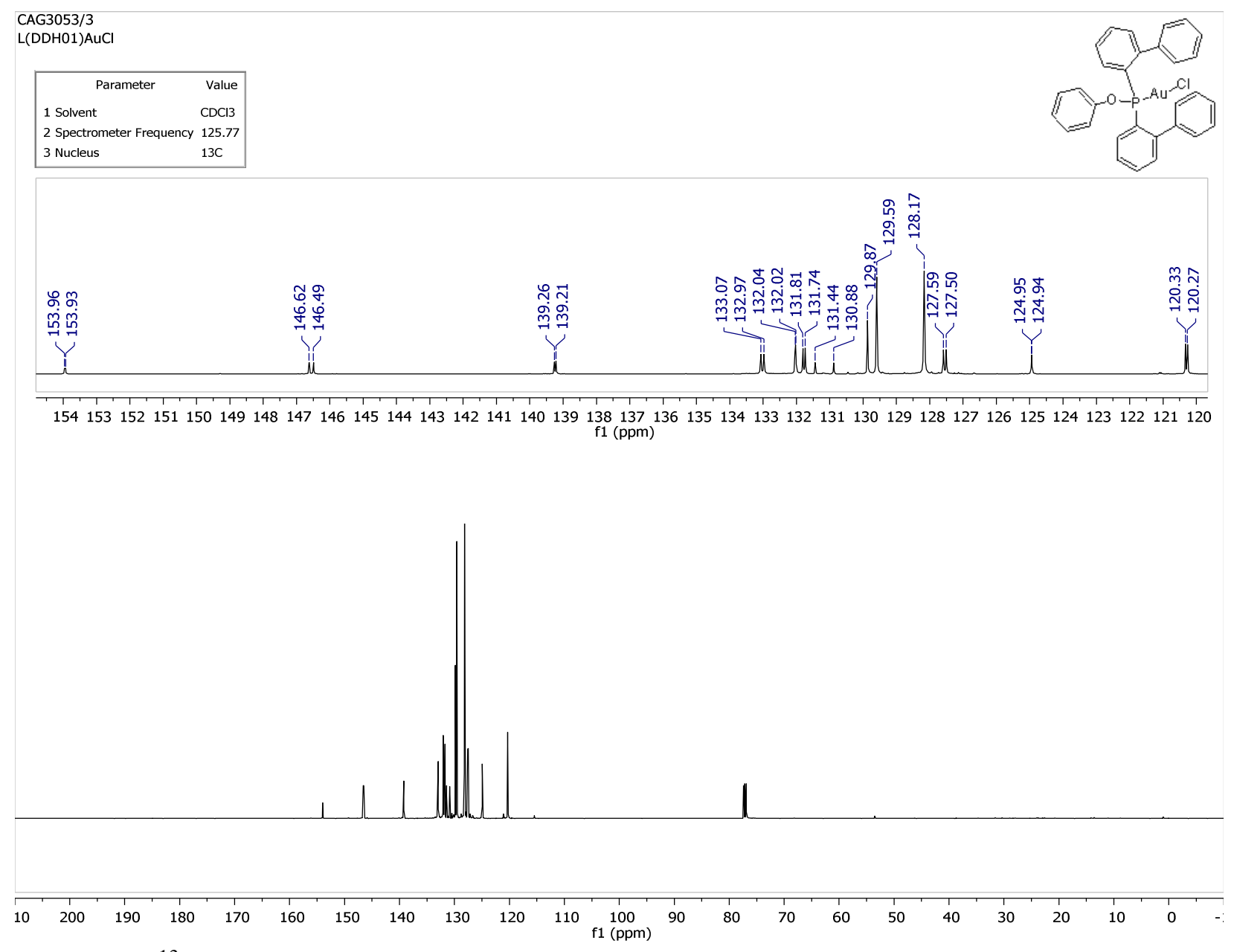

Figure S6. ${ }^{13} \mathrm{C}$ NMR Spectrum of $1 \mathbf{a}\left(125.77 \mathrm{MHz}, \mathrm{CDCl}_{3}\right)$. 


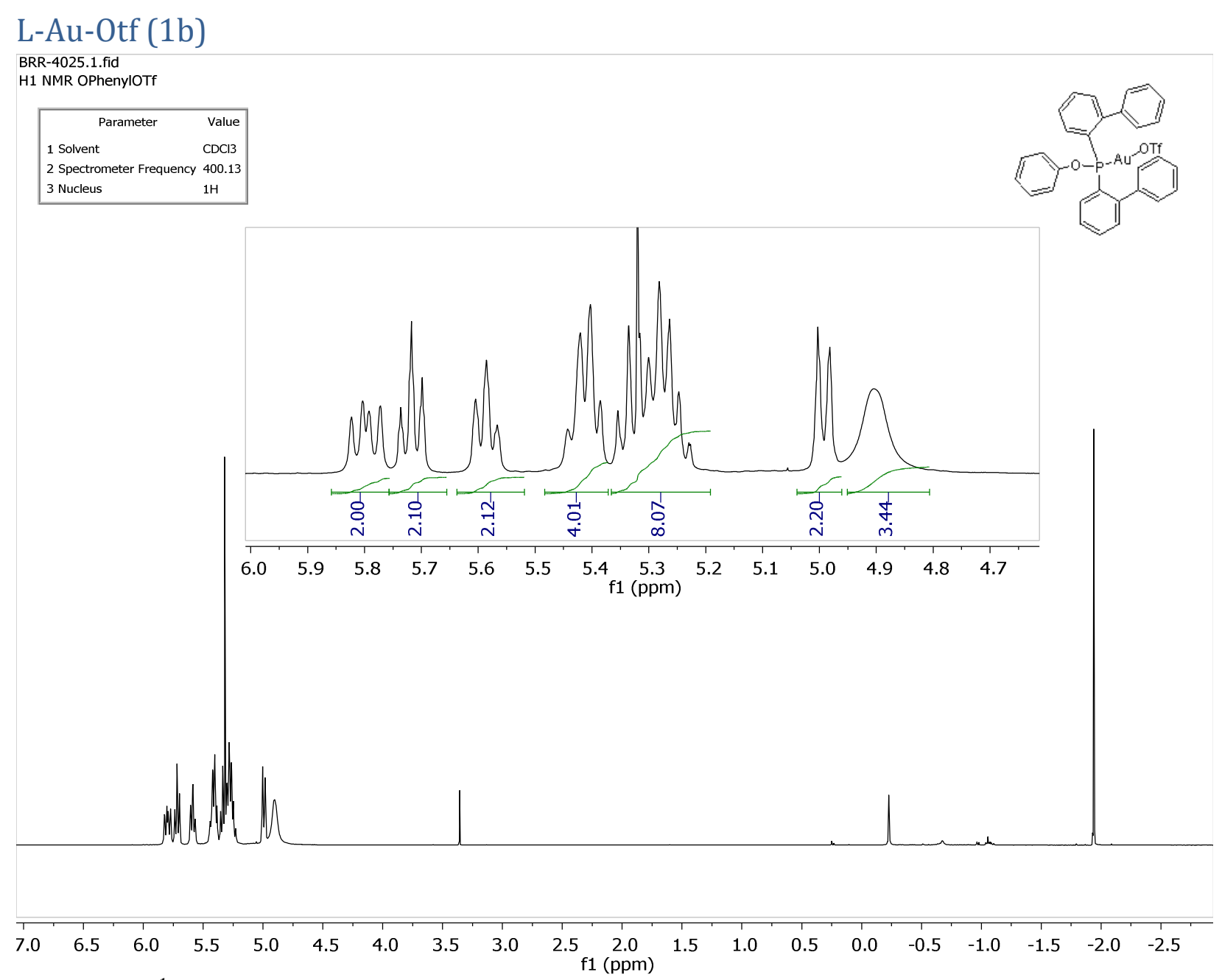

Figure S7. ${ }^{1} \mathrm{H}$ NMR Spectrum for $1 \mathbf{b}\left(400 \mathrm{MHz}^{\mathrm{CDCl}}\right)_{3}$.

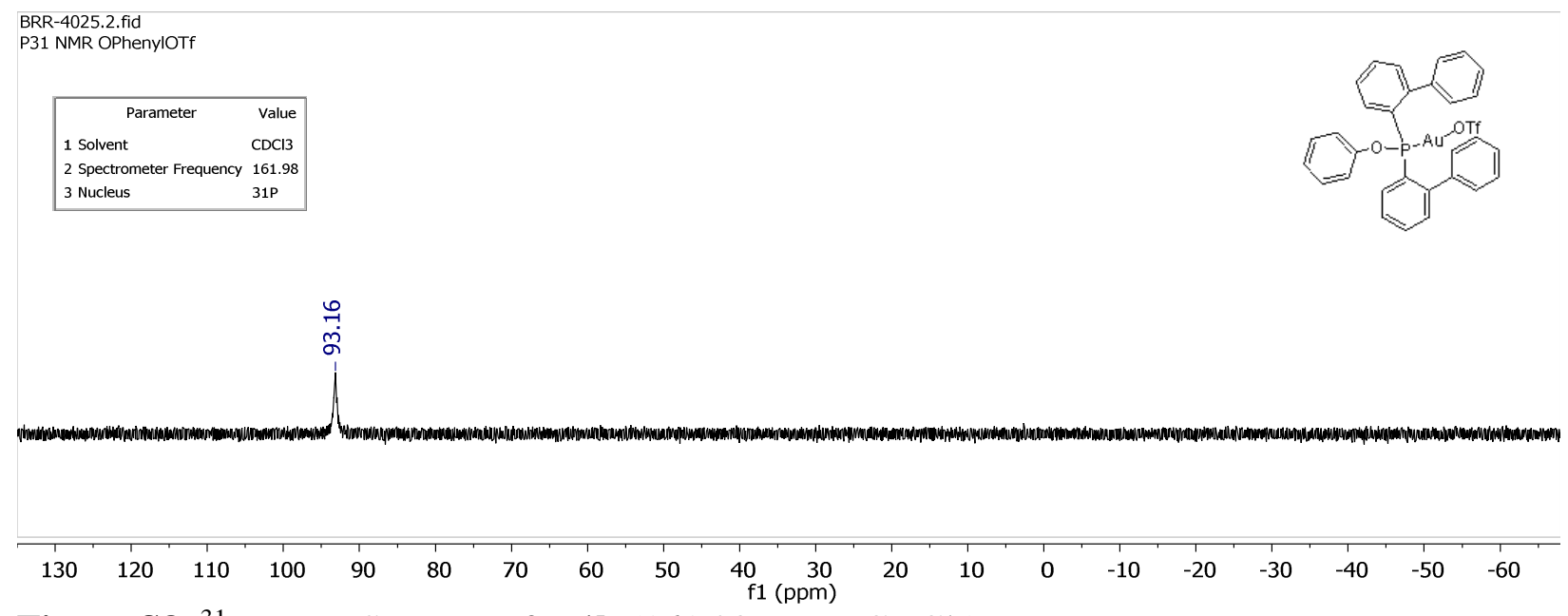

Figure S8. ${ }^{31} \mathrm{P}$ NMR Spectrum for $1 \mathbf{b}\left(161.98 \mathrm{MHz}, \mathrm{CDCl}_{3}\right)$. 
BRR-4025.6.fid

C13 NMR OphenylAuOTf

\begin{tabular}{|lr|}
\hline \multicolumn{1}{|c|}{ Parameter } & Value \\
1 Solvent & $\mathrm{CDCl} 3$ \\
2 Spectrometer & Frequency100.62 \\
3 Nucleus & $13 \mathrm{C}$ \\
\hline
\end{tabular}

\begin{tabular}{|c|c|}
\hline$\stackrel{\infty}{\infty}$ & ఫ \\
\hline & テ \\
\hline
\end{tabular}

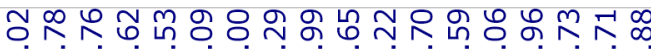

ติ

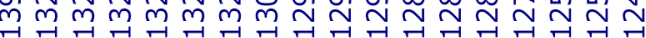

疍

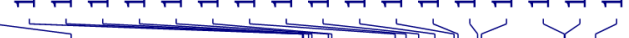

L

드유 욱

r

ir

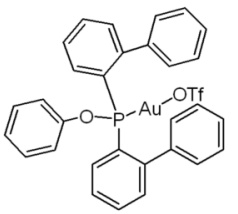

ข नु न

I

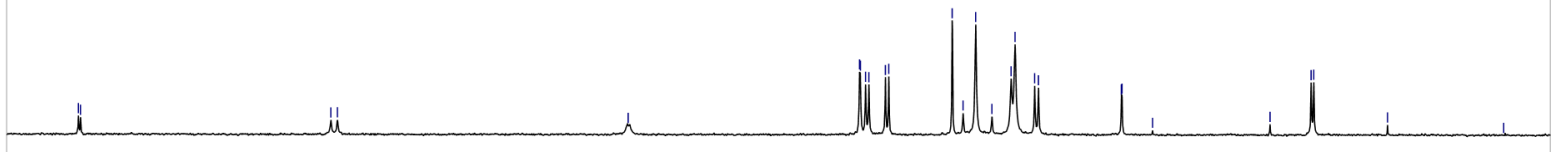

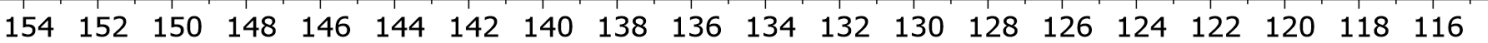
f1 (ppm)

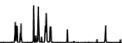

$\begin{array}{llllllllllllllllllllllllll}10 & 200 & 190 & 180 & 170 & 160 & 150 & 140 & 130 & 120 & 110 & 100 & 90 & 80 & 70 & 60 & 50 & 40 & 30 & 20 & 10 & 0\end{array}$

f1 (ppm)

Figure S9. ${ }^{13} \mathrm{C}$ NMR Spectrum for $\mathbf{1 b}\left(100.62 \mathrm{MHz}, \mathrm{CDCl}_{3}\right)$. 
Methoxypropene Complex (1c)

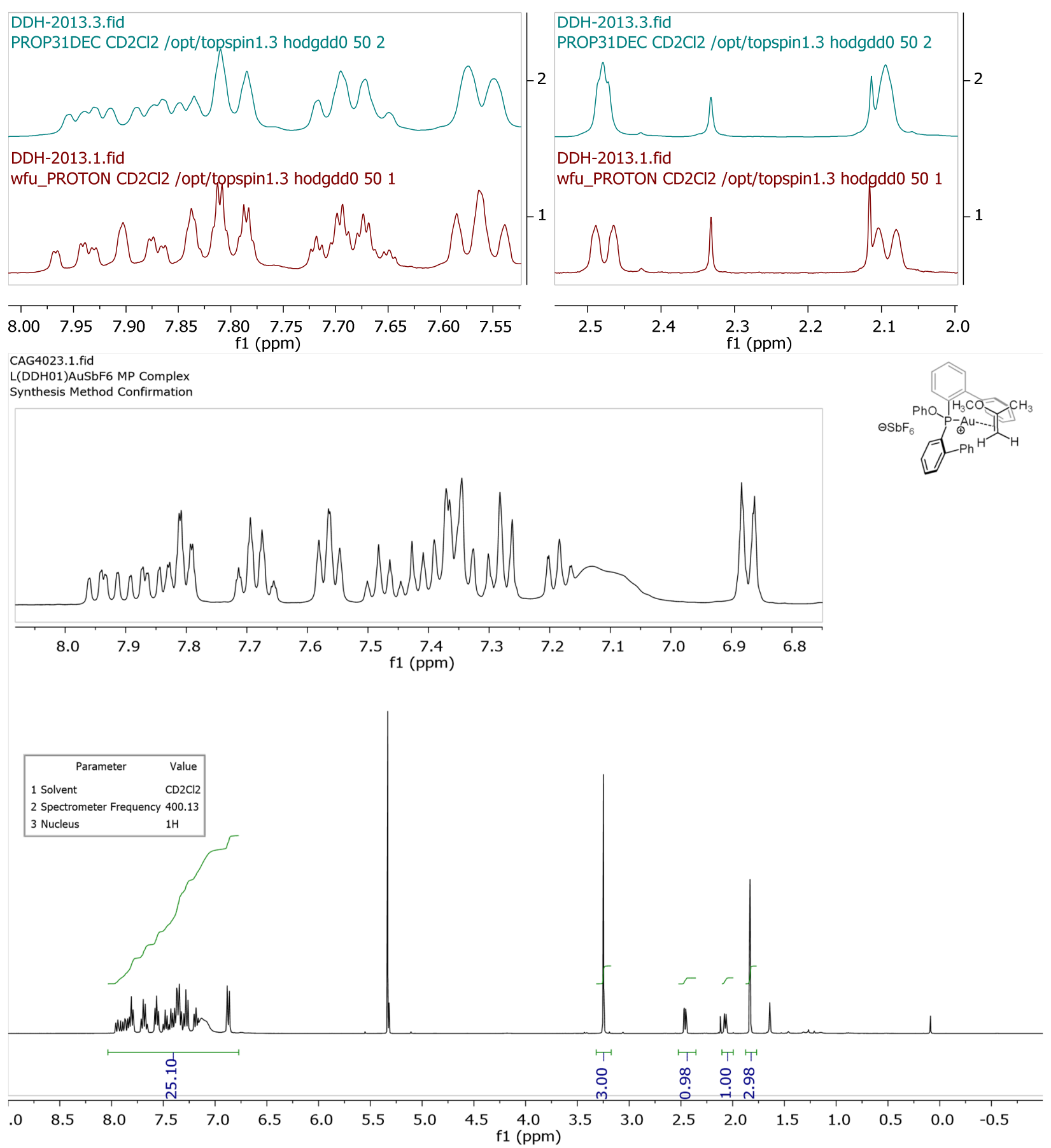

Figure S10. ${ }^{1} \mathrm{H}$ NMR Spectrum of $1 \mathrm{c}\left(400 \mathrm{MHz}, \mathrm{CD}_{2} \mathrm{Cl}_{2}\right)$ with above insets showing phosphorous decoupling. 


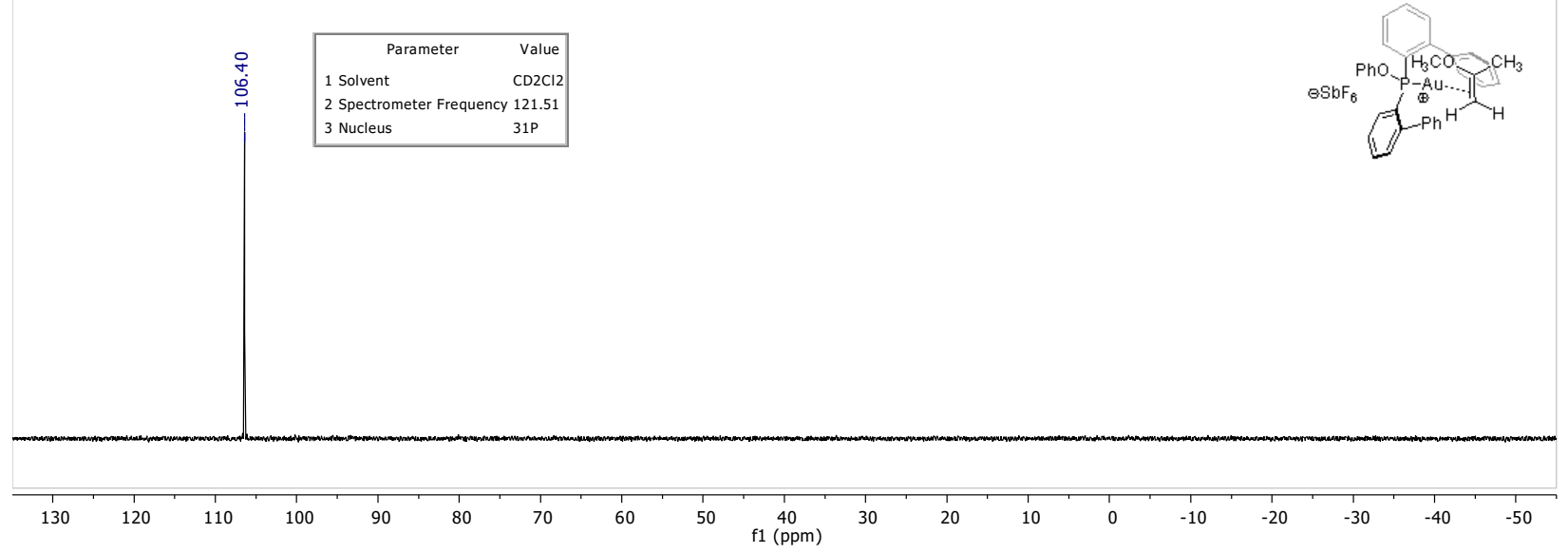

Figure S11. ${ }^{31} \mathrm{P}$ NMR Spectrum of $1 \mathbf{c}\left(400 \mathrm{MHz}, \mathrm{CD}_{2} \mathrm{Cl}_{2}\right)$.

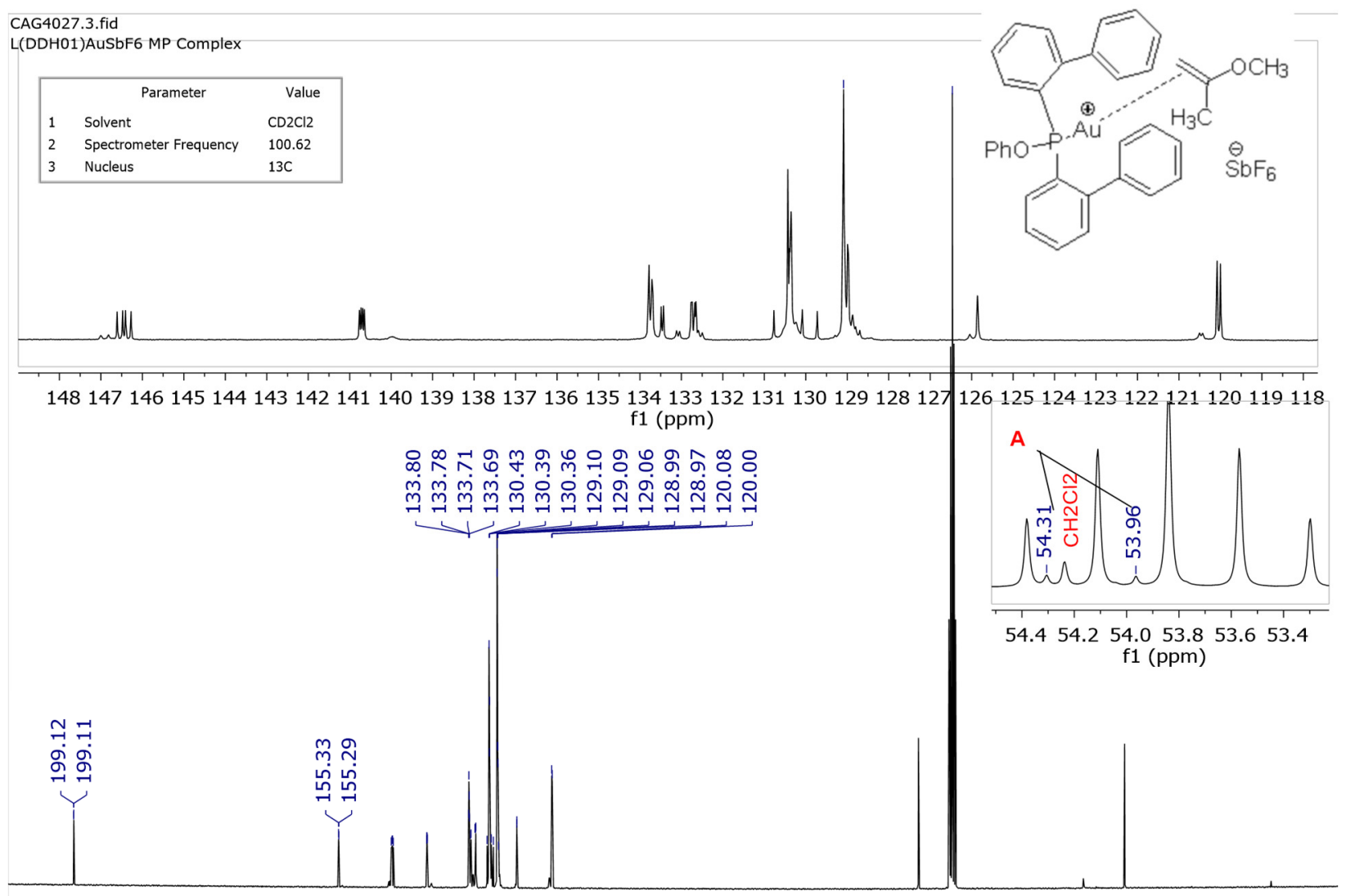

\begin{tabular}{|c|c|c|c|c|c|c|c|c|c|c|c|c|c|c|c|c|c|c|c|c|c|}
\hline \multirow[t]{2}{*}{10} & 200 & 190 & 180 & 170 & 160 & 150 & 140 & 130 & 120 & \multicolumn{2}{|c|}{$110 \quad 100$} & 90 & 80 & 70 & 60 & 50 & 40 & 30 & 20 & \multirow[t]{2}{*}{10} & \multirow[t]{2}{*}{0} \\
\hline & & 190 & & & & & & & 120 & & 1 (ppm & 90 & 80 & 10 & ou & 50 & 40 & 30 & 20 & & \\
\hline
\end{tabular}

Figure S12. ${ }^{13} \mathrm{C}$ NMR Spectrum of $1 \mathrm{c}\left(100.62 \mathrm{MHz}, \mathrm{CD}_{2} \mathrm{Cl}_{2}\right)$ additional peaks from hydrolysis of the enol ether. 


\section{Experimental Details and NMR Spectra for compounds with di([1,1'-biphenyl]-2- yl)(phenyl)phosphane, Ligand 2.}<smiles>c1ccc(-c2ccccc2P(c2ccccc2)c2ccccc2-c2ccccc2)cc1</smiles>

This compound was reported previously without full spectroscopic data. ${ }^{\text {SI }}$

di([1,1'-biphenyl]-2-yl)(phenyl)phosphane (2): n-Butyllithium (1.5 mL of a $2.5 \mathrm{M}$ solution in hexanes, $0.00375 \mathrm{~mol})$ was added to bromobiphenyl $(0.676 \mathrm{~g}, 0.0029 \mathrm{~mol})$ at $0^{\circ} \mathrm{C}$ in anhydrous diethyl ether $(20 \mathrm{ml})$. After $20 \mathrm{~min}, \mathrm{PhPCl}_{2}(0.264,0.0015 \mathrm{mmol})$ was added and the reaction warmed to room temperature and stirred overnight. After quenching with water $(20 \mathrm{~mL})$ the reaction was extracted with $3 \times 20 \mathrm{~mL}$ of dichloromethane. The combined organic solvents were dried with $\mathrm{Na}_{2} \mathrm{SO}_{4}$, decanted and concentrated in vacuo. The crude product could be purified by recrystallization using 1:2 $\mathrm{CH}_{2} \mathrm{Cl}_{2} / \mathrm{Hexanes}$ to yield a white solid $(0.35 \mathrm{~g}, 56 \%$ yield $)$.

${ }^{1} \mathbf{H}$ NMR (400 MHz, CD2 $\left.\mathbf{C l}_{2}\right): \delta 7.41(\mathrm{td}, J=7.2,1.1 \mathrm{~Hz}, 2 \mathrm{H}), 7.35-7.15(\mathrm{~m}, 15 \mathrm{H}), 7.11$ (dddd, $\mathrm{J}=7.6,3.5,1.4,0.5 \mathrm{~Hz}, 2 \mathrm{H}), 6.99(\mathrm{dm}, \mathrm{J}=8.2 \mathrm{~Hz}, 4 \mathrm{H})$.

${ }^{13}$ C NMR (126 MHz, CD2Cl2): $\delta 148.5\left(\mathrm{~d}, \mathrm{~J}_{\mathrm{CP}}=29.3 \mathrm{~Hz}\right), 142.4\left(\mathrm{~d}, \mathrm{~J}_{\mathrm{CP}}=6.2 \mathrm{~Hz}\right), 138.4\left(\mathrm{~d}, \mathrm{~J}_{\mathrm{CP}}\right.$ $=14 \mathrm{~Hz}), 136.9\left(\mathrm{~d}, \mathrm{~J}_{\mathrm{CP}}=15.3 \mathrm{~Hz}\right), 135.1,134.6\left(\mathrm{~d}, \mathrm{~J}_{\mathrm{CP}}=20.4 \mathrm{~Hz}\right), 130.6\left(\mathrm{~d}, \mathrm{~J}_{\mathrm{CP}}=4.8 \mathrm{~Hz}\right), 130.1$ $\left(\mathrm{d}, \mathrm{J}_{\mathrm{CP}}=3.7 \mathrm{~Hz}\right), 129.1,129.0,128.9\left(\mathrm{~d}, \mathrm{~J}_{\mathrm{CP}}=3.1 \mathrm{~Hz}\right), 128.0,127.9$, 127.5.

${ }^{31} \mathbf{P}\left\{{ }^{1} \mathbf{H}\right\}$ NMR (121.5 MHz, CDCl3): $\delta-20.2$.

(S1) Mitterhofer, F.; Schindlbauer, H. Monatsh. Chem. 1967, 98, 206-213. 
BRR-(Fredphos 2D).1.fid

H1 NMR Fredphos
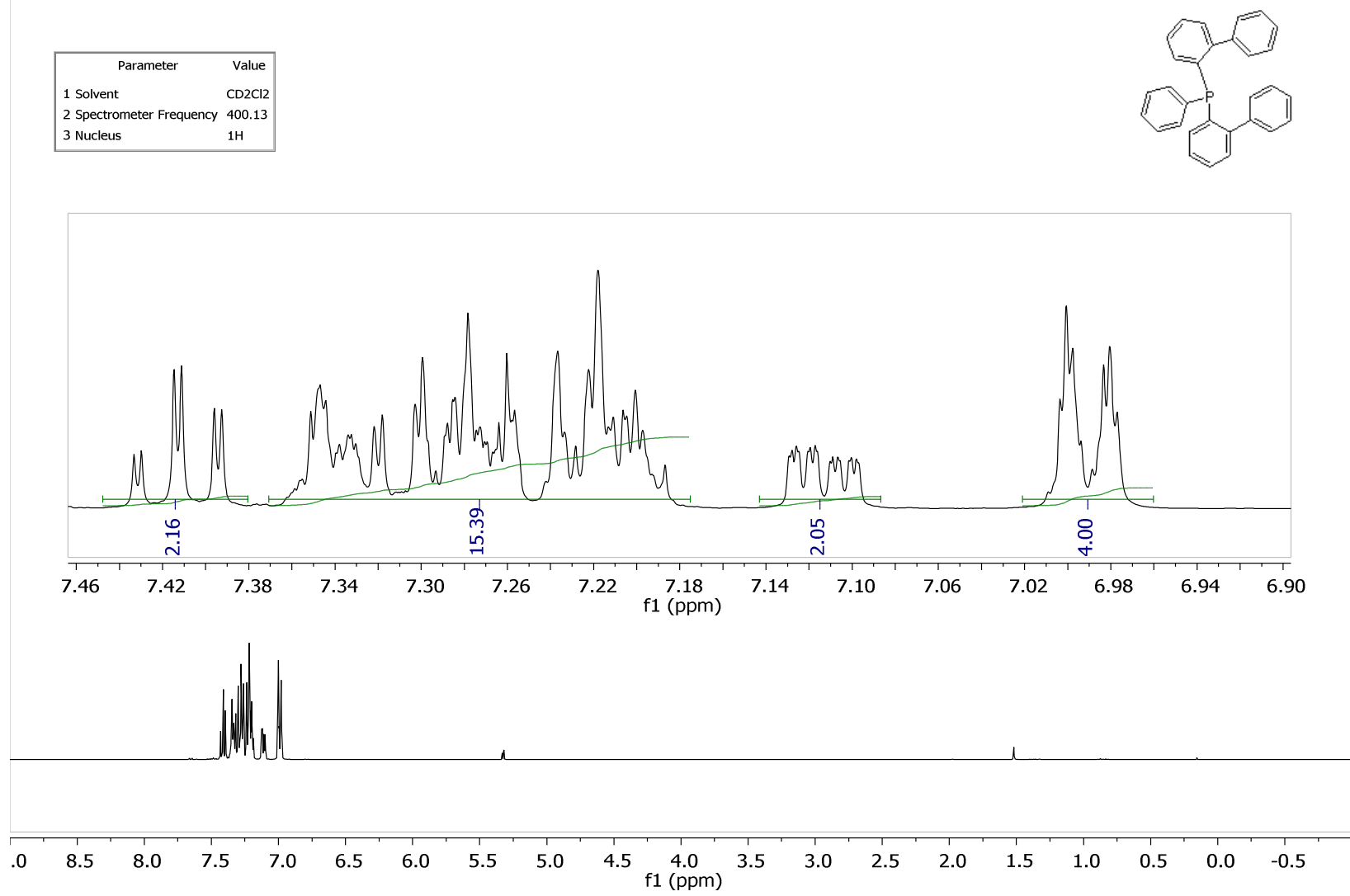

Figure S13. ${ }^{1} \mathrm{H}$ NMR spectrum of $2\left(400 \mathrm{MHz}, \mathrm{CD}_{2} \mathrm{Cl}_{2}\right)$.

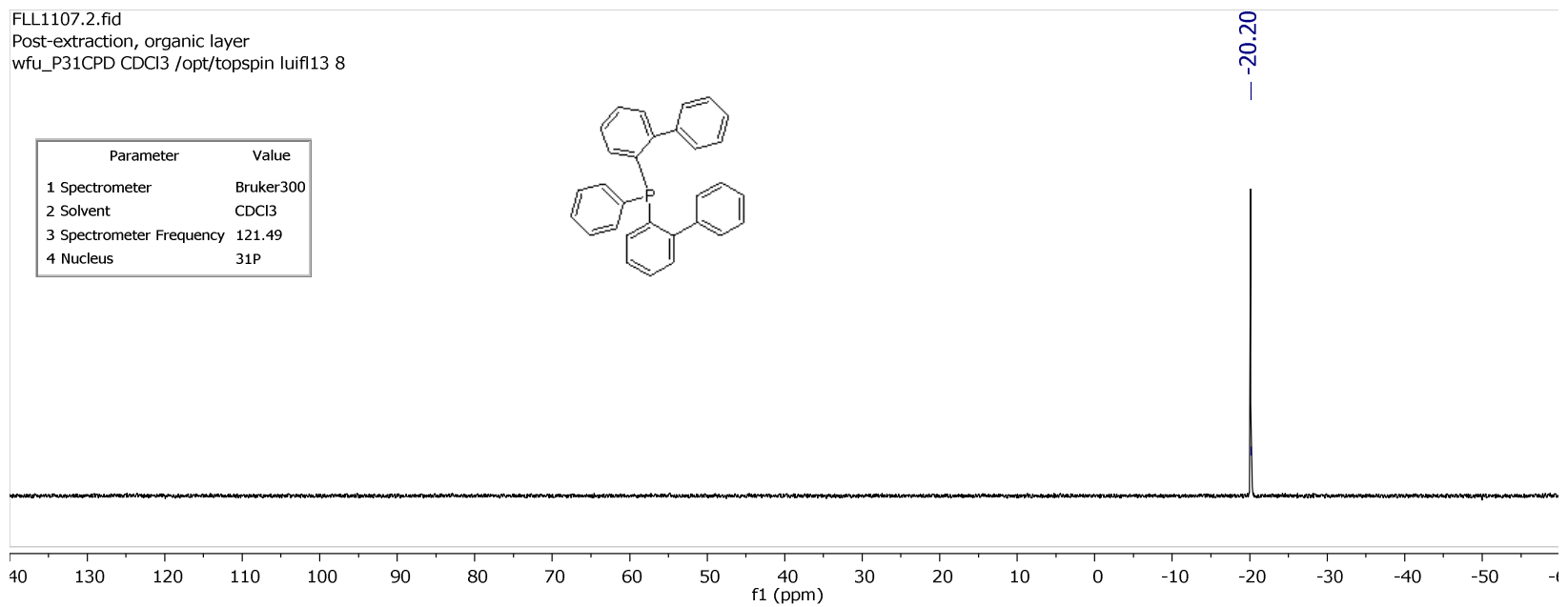

Figure S14. ${ }^{31} \mathrm{P}$ NMR Spectrum of $2\left(121.49 \mathrm{MHz}, \mathrm{CDCl}_{3}\right)$. 


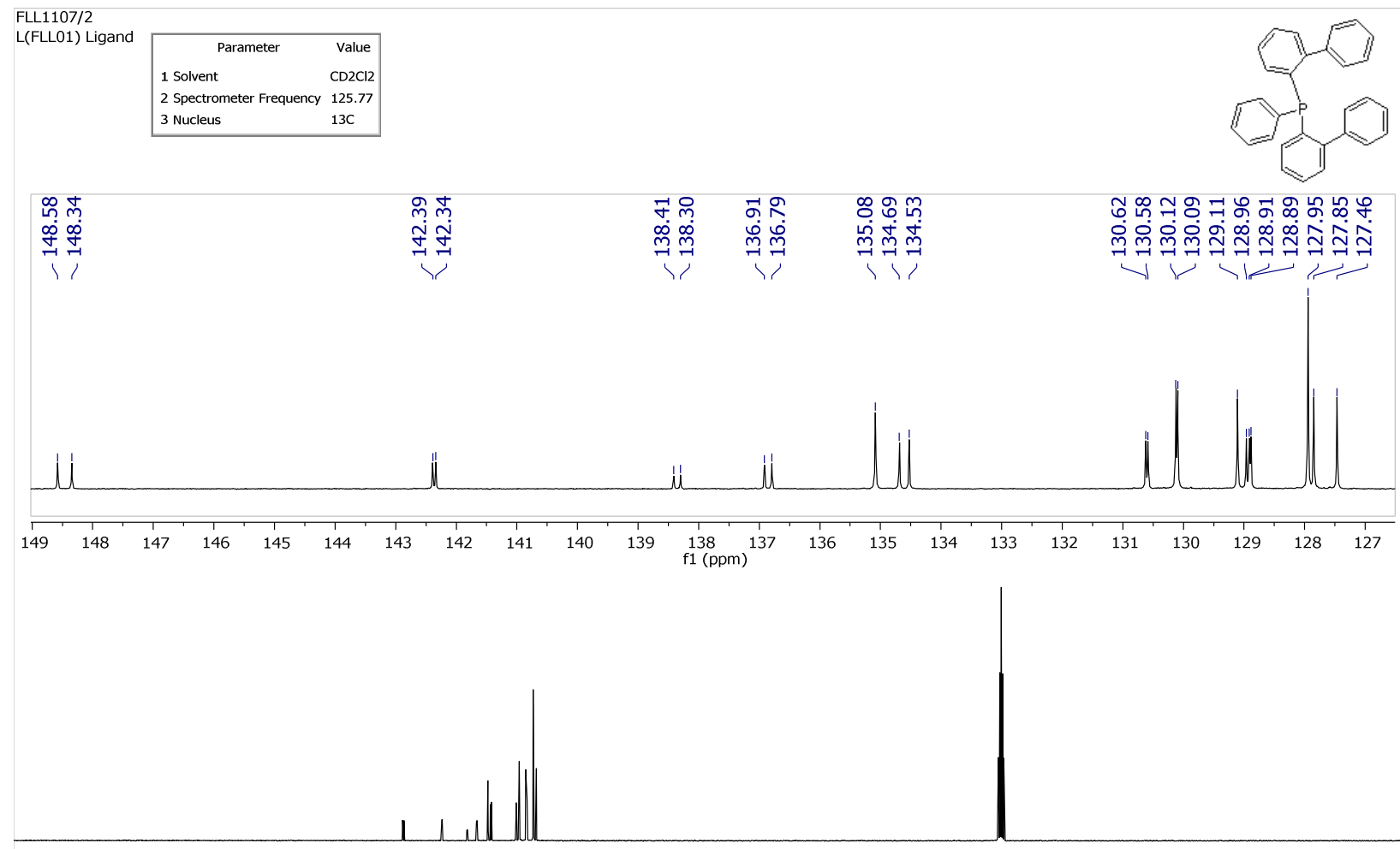

\begin{tabular}{llllllllllllllllllllllll}
\hline 10 & 200 & 190 & 180 & 170 & 160 & 150 & 140 & 130 & 120 & 110 & $\underset{\mathrm{f} 1}{100}(\mathrm{ppm})$ & 90 & 80 & 70 & 60 & 50 & 40 & 30 & 20 & 10 & 0 & -
\end{tabular}

Figure S15. ${ }^{13} \mathrm{C}$ NMR Spectrum of $2\left(157.77 \mathrm{MHz}, \mathrm{CD}_{2} \mathrm{Cl}_{2}\right)$. 
$\mathrm{L}-\mathrm{Au}-\mathrm{Cl}(2 \mathrm{a})$

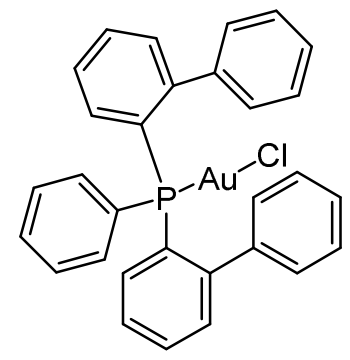

$\mathrm{L}-\mathrm{Au}-\mathrm{Cl}(2 \mathrm{a})$ was prepared according to the same synthesis as for $\mathrm{Ia}$ using $0.103 \mathrm{~g} \mathrm{Me} 2 \mathrm{SAuCl}$ $(0.35 \mathrm{mmol})$. White solid $(0.142 \mathrm{~g}, 63 \%$ yield $)$.

${ }^{1}$ H NMR (500 MHz, CD2Cl2): $7.60(\mathrm{~m}, 5 \mathrm{H}), 7.51(\mathrm{t}, \mathrm{J}=7.4 \mathrm{~Hz}, 2 \mathrm{H}), 7.41(\mathrm{t}, \mathrm{J}=7.2 \mathrm{~Hz}, 2 \mathrm{H}), 7.37$ $(\mathrm{m}, 2 \mathrm{H}), 7.34(\mathrm{t}, \mathrm{J}=7.2 \mathrm{~Hz}, 2 \mathrm{H}), 7.18(\mathrm{~m}, 6 \mathrm{H}), 6.82(\mathrm{~d}, \mathrm{~J}=7.21 \mathrm{~Hz}, 4 \mathrm{H})$.

${ }^{13}$ C NMR (125.77 MHz, CD $\left.\mathbf{C l}_{2}\right)$ : 148.0 (d, J=15.5 Hz), 140.9 (d, J=6.8 Hz), 135.5 (d, J=14.5 Hz), 134.9 (d, J=6.0 Hz), 132.7 (d, J=8.4 Hz), 132.3 (d, J=2.3 Hz), 131.7 (d, J=2.3 Hz), 131 (d, $\mathrm{J}=61.5 \mathrm{~Hz}$ ), 130.1, 129.7 (d, J=11.7 Hz), 129.3 (d, J=61.9), 128.6, 128.5, 128.1 (d, J=8.5 Hz).

\section{${ }^{31} \mathbf{P}\left\{{ }^{1} \mathrm{H}\right\}$ NMR (121.49MHz, CDCl3): $\delta 17.7$}

Elemental Analysis Calcd (found) C, 55.70 (55.81); H, 3.58 (3.90). (Corresponding batch yield not recorded). 
FLL1101/1

FLL1101 \& FLL1103 Mo. Liqu. 2nd recryst. L(FLL01)AuCl

\begin{tabular}{|lr|}
\hline \multicolumn{1}{|c|}{ Parameter } & Value \\
1 Solvent & $\mathrm{CD} 2 \mathrm{Cl} 2$ \\
2 Spectrometer Frequenieg.13 \\
3 Nucleus & $1 \mathrm{H}$ \\
\hline
\end{tabular}
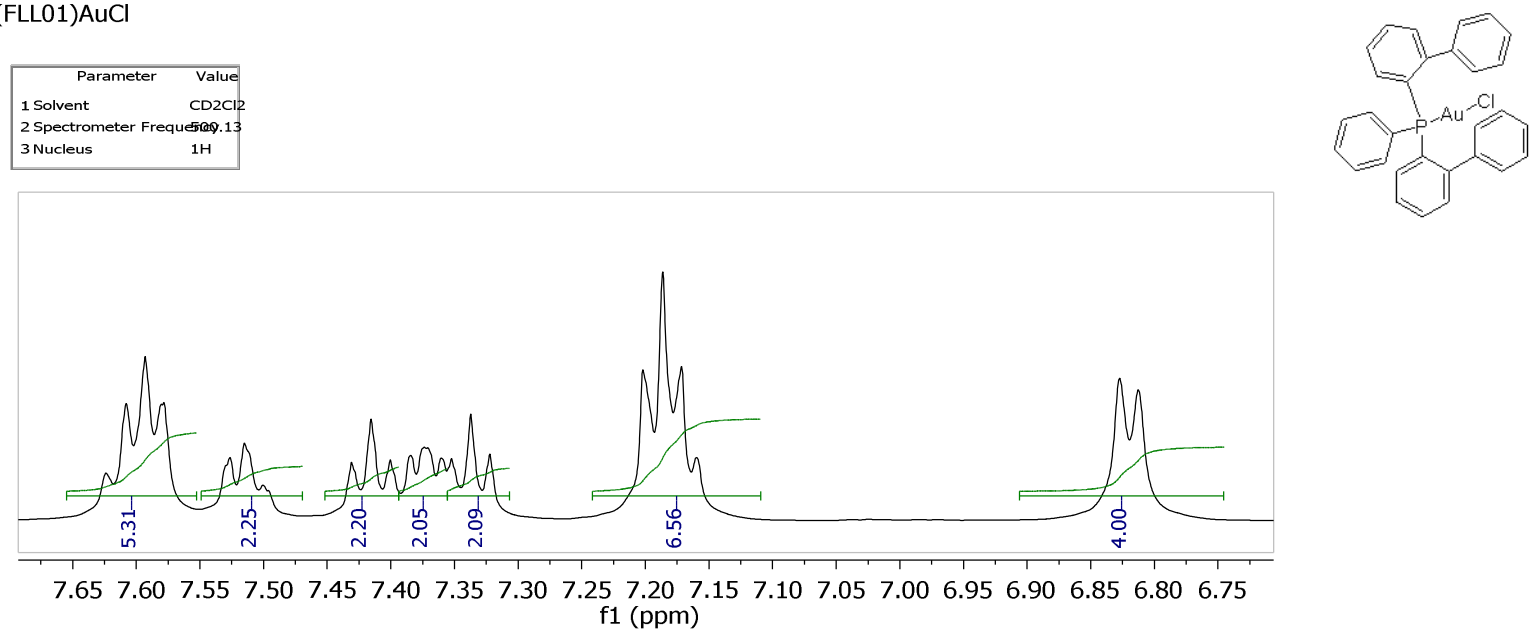

f1 (ppm)

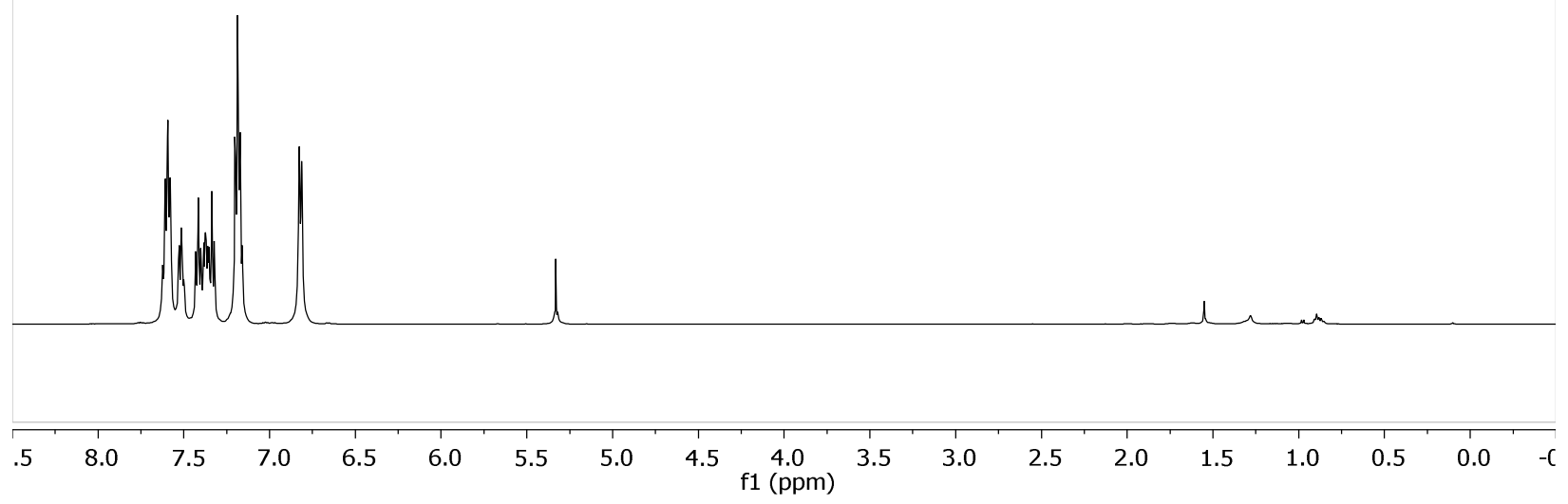

Figure S16. ${ }^{1} \mathrm{H}$ NMR spectrum for $2 \mathbf{2 a}\left(500 \mathrm{MHz}, \mathrm{CD}_{2} \mathrm{Cl}_{2}\right)$.

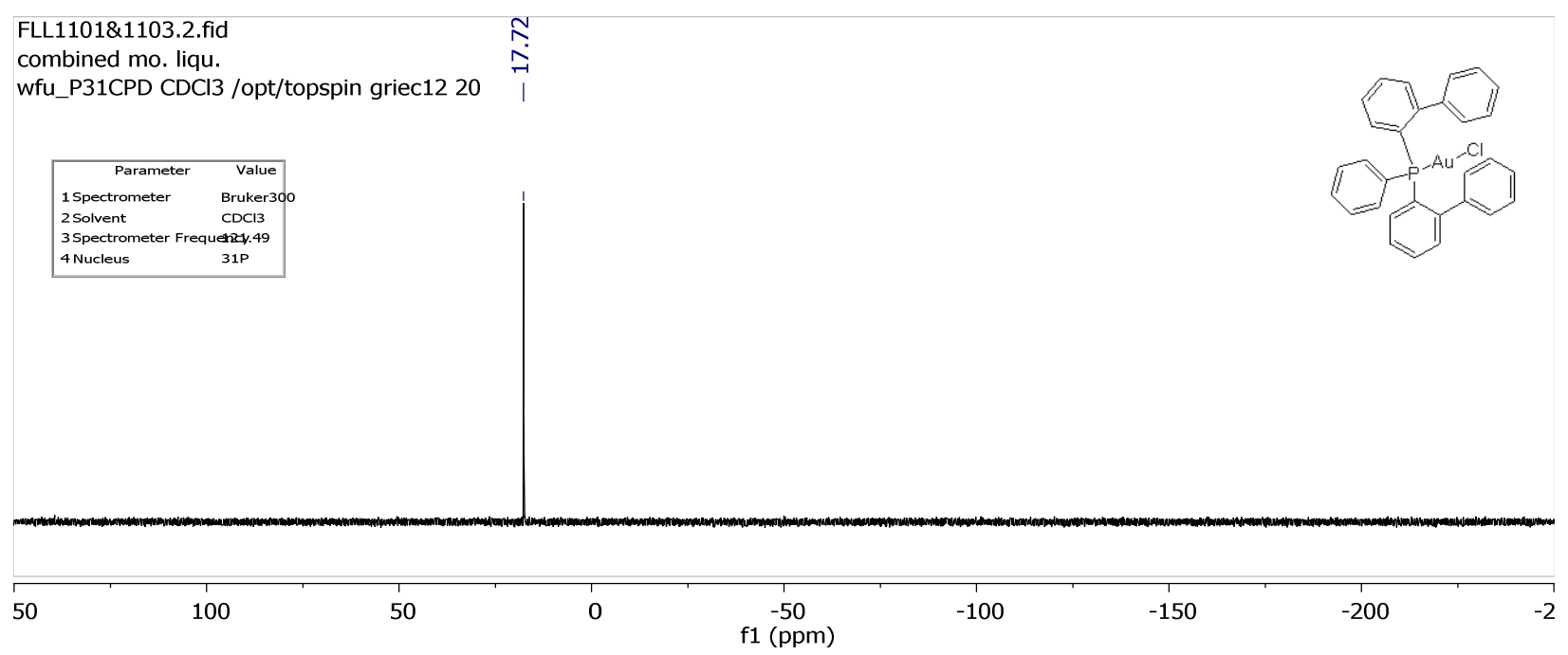

Figure S17. ${ }^{31} \mathrm{P}$ NMR Spectrum for 2a $\left(121.49, \mathrm{CDCl}_{3}\right)$. 
FLL1101/2

FLL1101 \& FLL1103 Mo. Liqu. 2nd recryst. L(FLL01)AuCl

\begin{tabular}{|c|c|c|}
\hline & Parameter & Value \\
\hline 1 & Solvent & $\mathrm{CD} 2 \mathrm{Cl} 2$ \\
\hline 2 & Spectrometer Frequency & 125.77 \\
\hline 3 & Nucleus & $13 \mathrm{C}$ \\
\hline
\end{tabular}

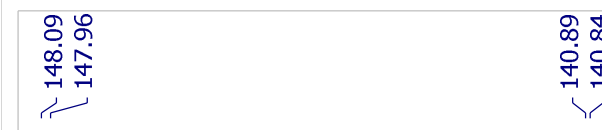

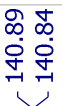

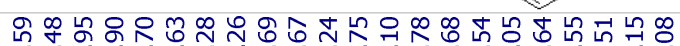
崩

1
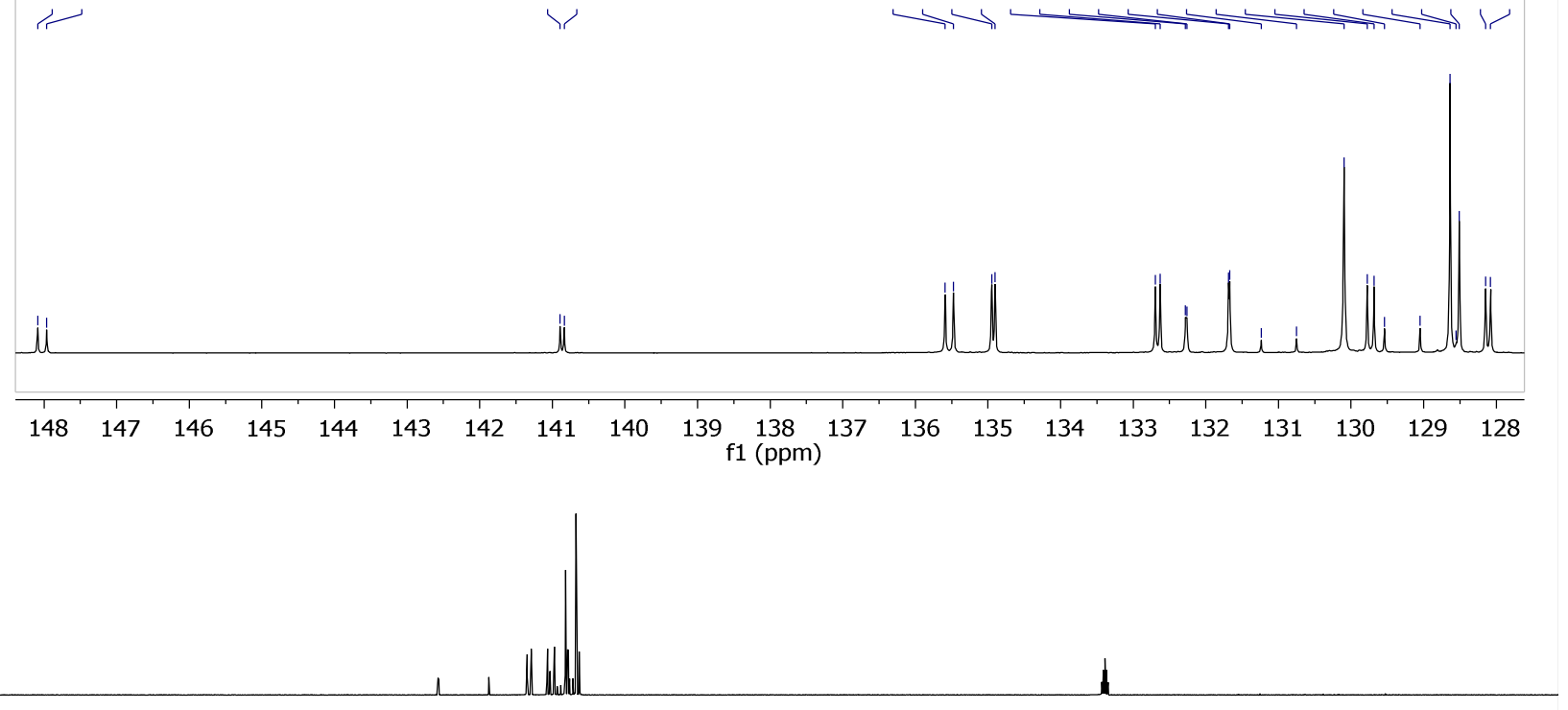

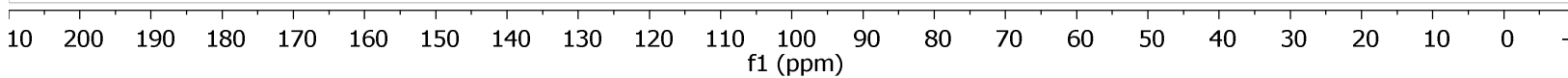

Figure S18. ${ }^{13} \mathrm{C}$ NMR Spectrum for $\mathbf{2 a}\left(125.77 \mathrm{MHz}, \mathrm{CD}_{2} \mathrm{Cl}_{2}\right)$. 


\section{L-Au-OTf (2b)}<smiles>c1ccc(-c2ccccc2[PH](c2ccccc2)(c2ccccc2)c2ccccc2-c2ccccc2)cc1</smiles>

Gold triflate $\mathbf{2 b}$ was prepared according to the same procedure as $\mathbf{1 b}$. The crude product was recrystallized from 1:2 $\mathrm{CH}_{2} \mathrm{Cl}_{2} / \mathrm{Hexanes}$ to give pure product $(0.12 \mathrm{~g}, 100 \%)$.

${ }^{1} \mathbf{H}$ NMR (400 MHz, CDCl $): \delta 7.61(\mathrm{t}, \mathrm{J}=6.8 \mathrm{~Hz}, 3 \mathrm{H}), 7.53(\mathrm{~m}, 4 \mathrm{H}), 7.43(\mathrm{t}, \mathrm{J}=7.4 \mathrm{~Hz}, 2 \mathrm{H})$, 7.37 (m, 4H), $7.22(\mathrm{t}, \mathrm{J}=7.7 \mathrm{~Hz}, 4 \mathrm{H}), 7.12\left(\mathrm{dd}, \mathrm{J}_{\mathrm{HP}}=11.5, \mathrm{~J}_{\mathrm{HH}}=7.7 \mathrm{H}, 2 \mathrm{H}\right), 6.75$ (br s, 4H).

${ }^{13}$ C NMR (101 MHz, CD $\left.2 \mathbf{C l}_{2}\right): \delta 147.67,140.68,135.21,135.07,134.62,132.86,132.83$, 132.62, 132.33, 130.00, 129.87, 129.24, 128.70, 128.41, 127.70, 127.03, 125.45, 122.28, 119.11, $115.94,120.70\left(\mathrm{q}, \mathrm{J}_{\mathrm{CF}}=319.1 \mathrm{~Hz}\right)$. Significant line-broadening in most signals resulted in overrepresentation of hexanes impurities which were minor by ${ }^{l} H$ NMR spectroscopy.

${ }^{31}$ P NMR (162 MHz, CDCl3): $\delta 9.4$

Elemental Analysis Calcd (found) for $\mathrm{C}_{31} \mathrm{H}_{23} \mathrm{AuF}_{3} \mathrm{O}_{3} \mathrm{PS}$ : C, 48.96 (48.51); H, 3.05 (3.20). (Corresponding batch yield not recorded.) 

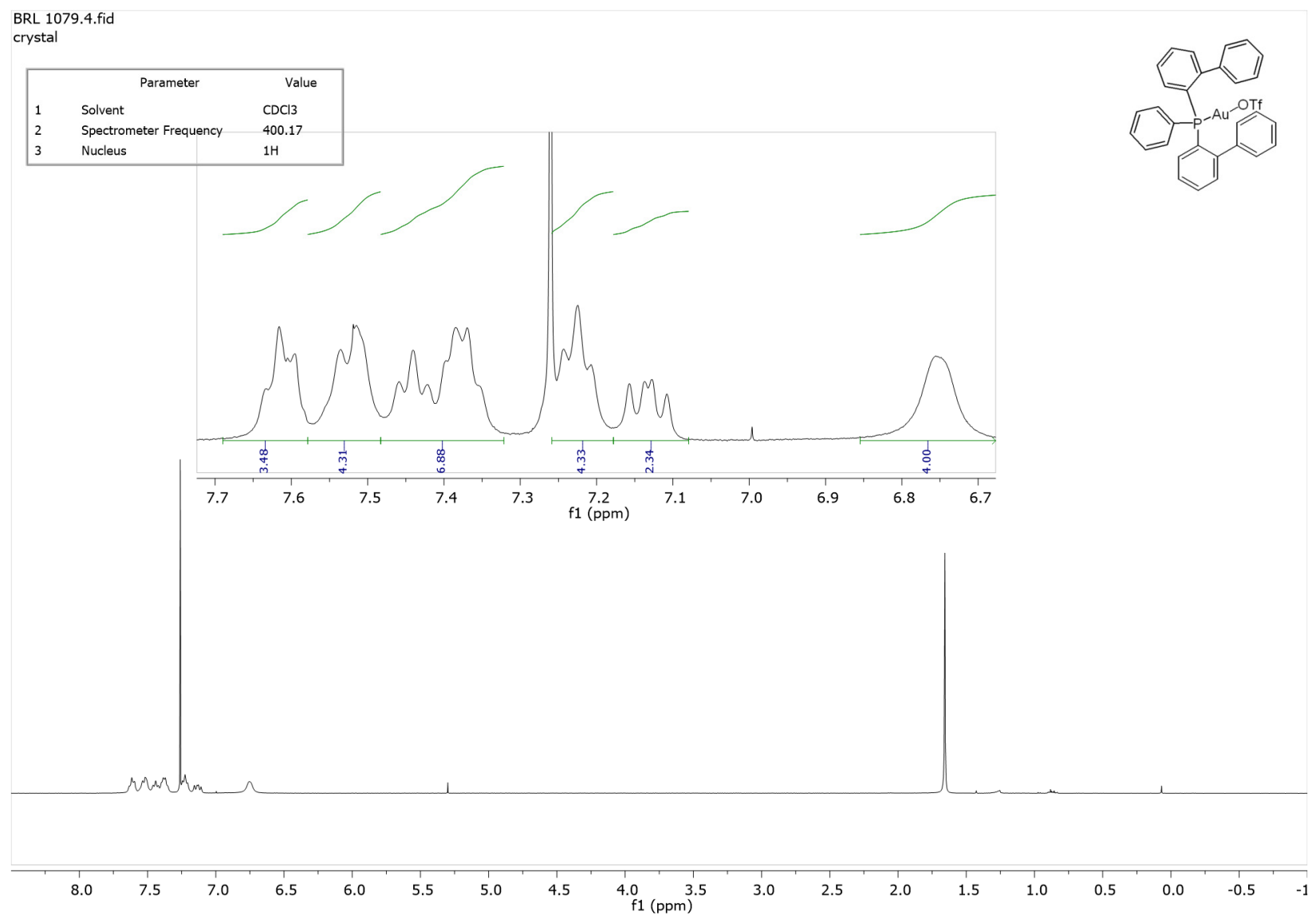

Figure S19. ${ }^{1} \mathrm{H}$ NMR for $\mathbf{2 b}\left(400.17 \mathrm{MHz}, \mathrm{CDCl}_{3}\right)$.
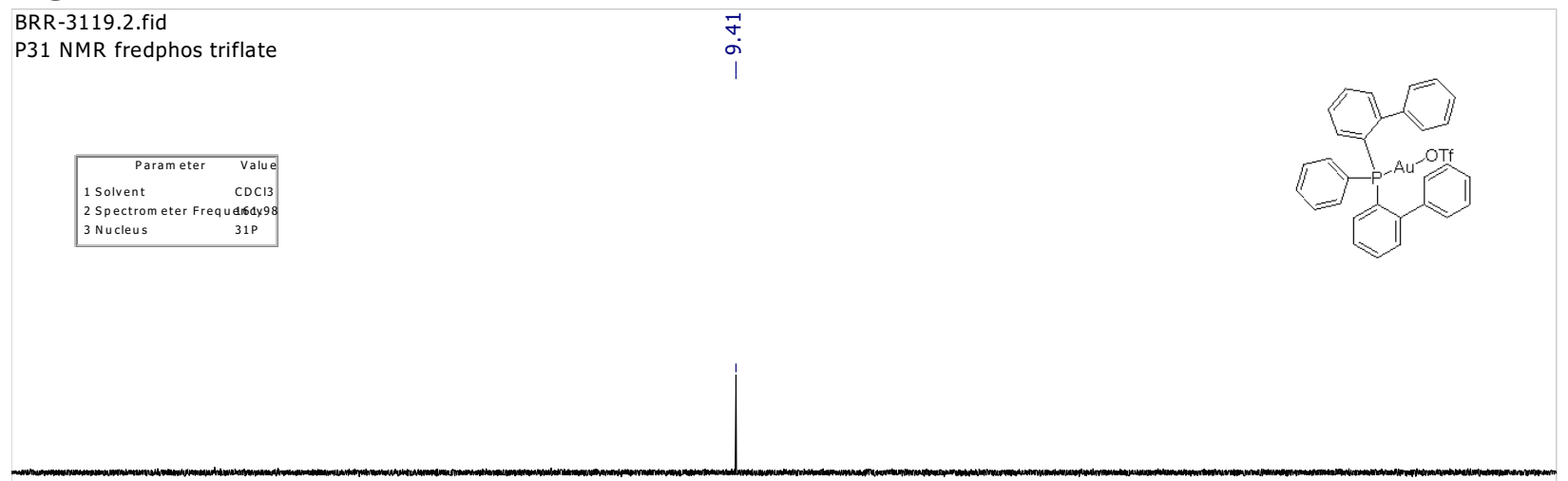

\begin{tabular}{|c|c|c|c|c|c|c|c|c|c|c|c|c|c|c|c|}
\hline 1 & 11 & 1 & & $T$ & 1 & 1 & 1 & 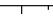 & 1 & 1 & 11 & 1 & 11 & 71 & 1 \\
\hline .50 & 130 & 110 & 90 & 70 & 50 & 30 & & $\mathrm{n}^{-10}$ & -30 & -50 & -70 & -90 & -110 & -130 & -150 \\
\hline
\end{tabular}

Figure S20. ${ }^{31} \mathrm{P}$ NMR for $\mathbf{2 b}\left(161.98 \mathrm{MHz}, \mathrm{CDCl}_{3}\right)$. 


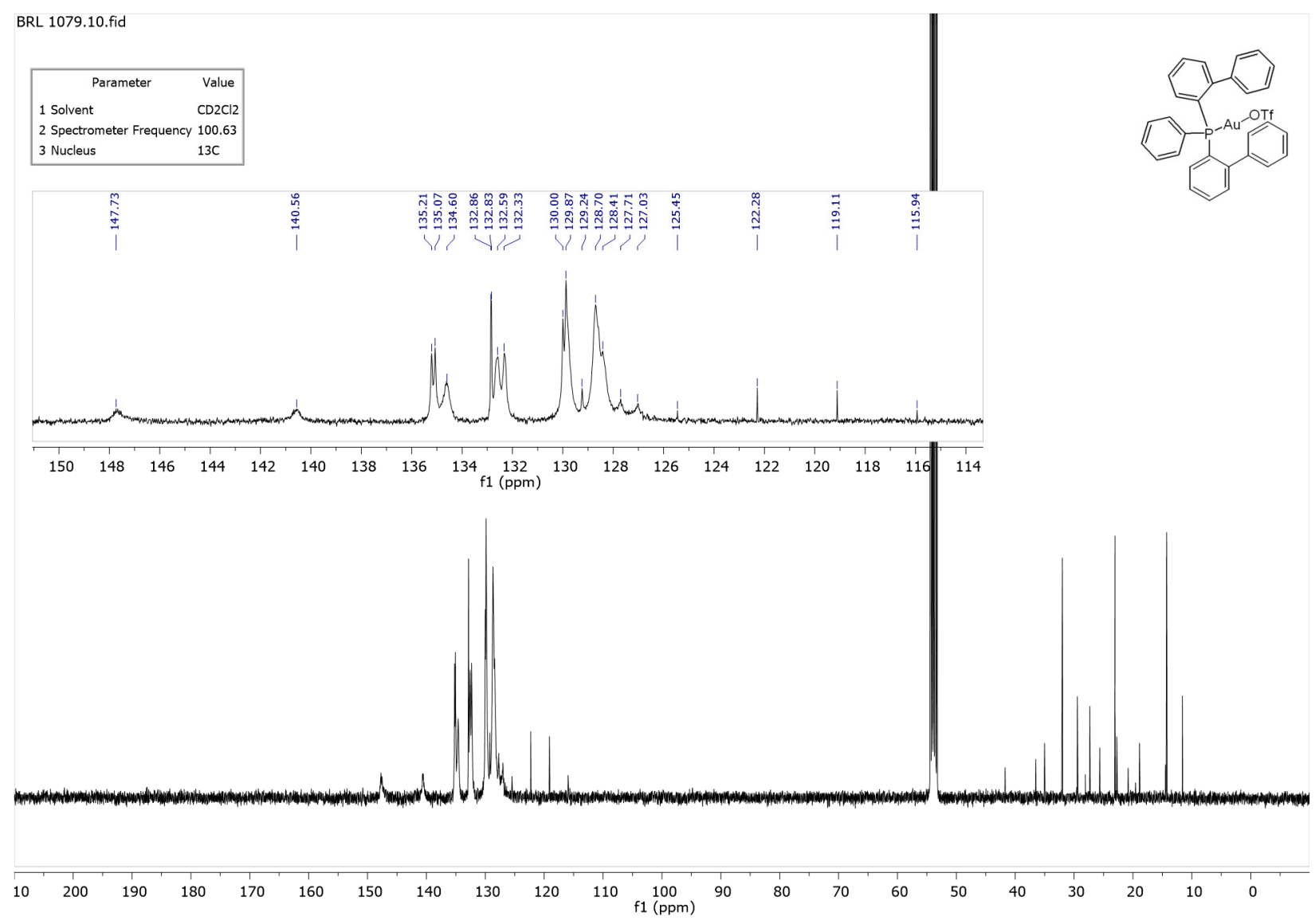

Figure S21. ${ }^{13} \mathrm{C}$ NMR spectrum for $2 \mathbf{b}\left(100.63 \mathrm{MHz}, \mathrm{CD}_{2} \mathrm{Cl}_{2}\right)$ (upfield impurities are hexanes isomers). 


\section{Methoxypropene Complex (2c)}

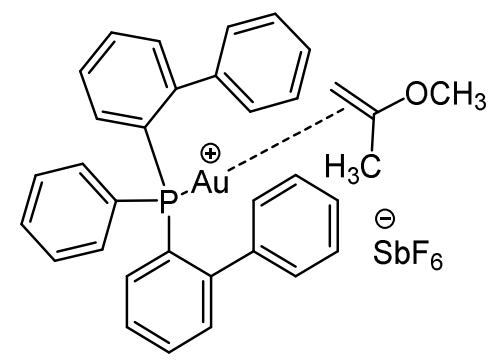

Prepared according to the same procedure as $1 \mathrm{c}$ using $0.058 \mathrm{~g} \mathrm{~L}-\mathrm{Au}-\mathrm{Cl}(0.09 \mathrm{mmol})$ to yield white solid (0.082 g, 95.7\% yield).

${ }^{1} \mathbf{H}$ NMR (400 MHz, $\left.\mathbf{C D}_{2} \mathbf{C l}_{2}\right): \delta 7.75-7.65(\mathrm{~m}, 3 \mathrm{H}), 7.62-7.39(\mathrm{~m}, 10 \mathrm{H}), 7.32-7.21(\mathrm{~m}$, $6 \mathrm{H}), 6.89(\mathrm{~d}, \mathrm{~J}=7.11 \mathrm{~Hz}, 4 \mathrm{H}), 3.6(\mathrm{~s}, 3 \mathrm{H}), 2.65(\mathrm{dd}, \mathrm{J}=6.7,1.7 \mathrm{~Hz}, 1 \mathrm{H}), 2.27$ (dq, J=6.6, 0.7 Hz), $2.15(\mathrm{~s}, 3 \mathrm{H})$.

${ }^{13} \mathrm{C}$ NMR (100 MHz, $\mathrm{CD}_{2} \mathrm{Cl}_{2}$, Peaks in bold are coordinated methoxypropene): 197.93, 146.86 (d, J=16.6 Hz), 146.82 (d, J=16.3 Hz), 141.51 (d, J = 7.4 Hz), 141.42 (d, J = 7.8 Hz), 135.50 (d, J $=5.5 \mathrm{~Hz}), 135.42(\mathrm{~d}, \mathrm{~J}=5.9 \mathrm{~Hz}), 134.73(\mathrm{~d}, \mathrm{~J}=14.5 \mathrm{~Hz}), 133.03(\mathrm{br} \mathrm{d}, \mathrm{J}=8.5 \mathrm{~Hz}), 132.93(\mathrm{~d}, \mathrm{~J}=$ $2.6 \mathrm{~Hz}), 132.60(\mathrm{~d}, \mathrm{~J}=2.9 \mathrm{~Hz}), 132.56(\mathrm{~d}, \mathrm{~J}=2.4 \mathrm{~Hz}), 130.24(\mathrm{~d}, \mathrm{~J}=12.0 \mathrm{~Hz}), 130.16,130.07$, 129.58 (d, J = 60.6 Hz), 128.85, 128.84, 128.84 (d, J=8.3 Hz), 127.77 (d, J=60.7 Hz), 127.69 (d, J=60.3 Hz), 59.68, 54.69 (d, J = 30.7 Hz), 25.52.

\section{${ }^{31} \mathbf{P}$ NMR (162 MHz, $\left.\mathbf{C D}_{2} \mathrm{Cl}_{2}\right): \delta 22.3 \mathrm{ppm}$}

Elemental Analysis Calcd (found) for $\mathrm{C}_{34} \mathrm{H}_{31} \mathrm{AuF}_{6} \mathrm{OPSb}, \mathrm{C}, 44.42$ (45.49), H, 3.40 (3.95). Although these results are outside the range viewed as establishing analytical purity, they are provided to illustrate the best values obtained to date. (Corresponding batch yield, $45.5 \mathrm{mg}$, $64 \%)$ 


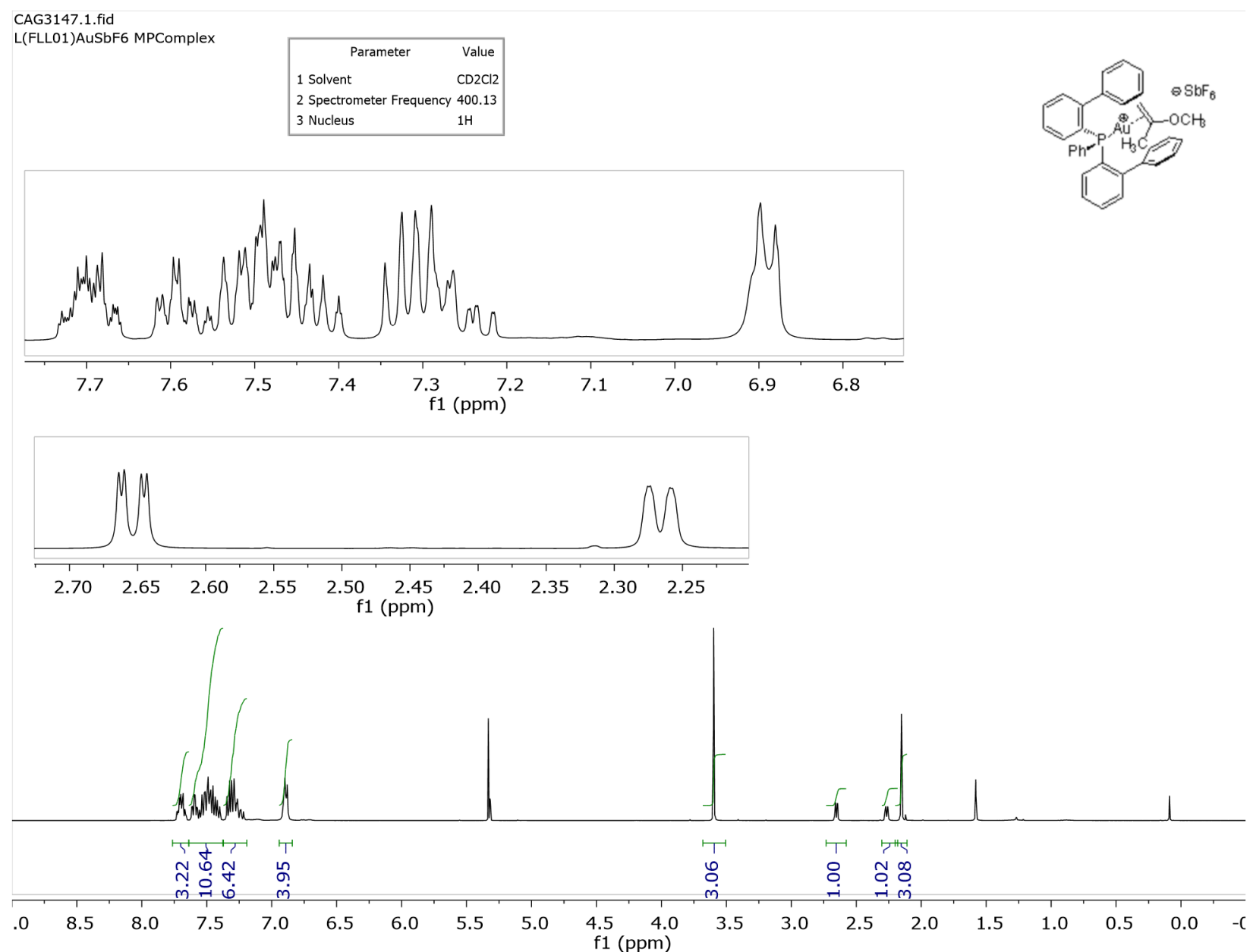

Figure S22. ${ }^{1} \mathrm{H}$ NMR Spectrum of $2 \mathbf{c}\left(400.13 \mathrm{MHz}, \mathrm{CD}_{2} \mathrm{Cl}_{2}\right)$.

CAG3147.2.fid

L(FLL01)AuSbF6 MPComplex

ก

\begin{tabular}{|ll|}
\hline \multicolumn{1}{|c}{ Parameter } & \multicolumn{1}{c|}{ Value } \\
1 Solvent & $\mathrm{CD} 2 \mathrm{Cl} 2$ \\
2 Spectrometer Frequency & 161.98 \\
3 Nucleus & $31 \mathrm{P}$ \\
\hline
\end{tabular}

$\stackrel{N}{N}$

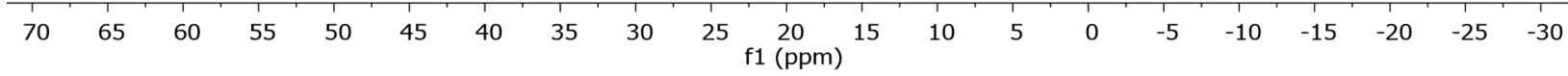

Figure S23. ${ }^{31} \mathrm{P}$ NMR Spectrum of $2 \mathbf{c}\left(161.98 \mathrm{MHz}, \mathrm{CD}_{2} \mathrm{Cl}_{2}\right)$. 


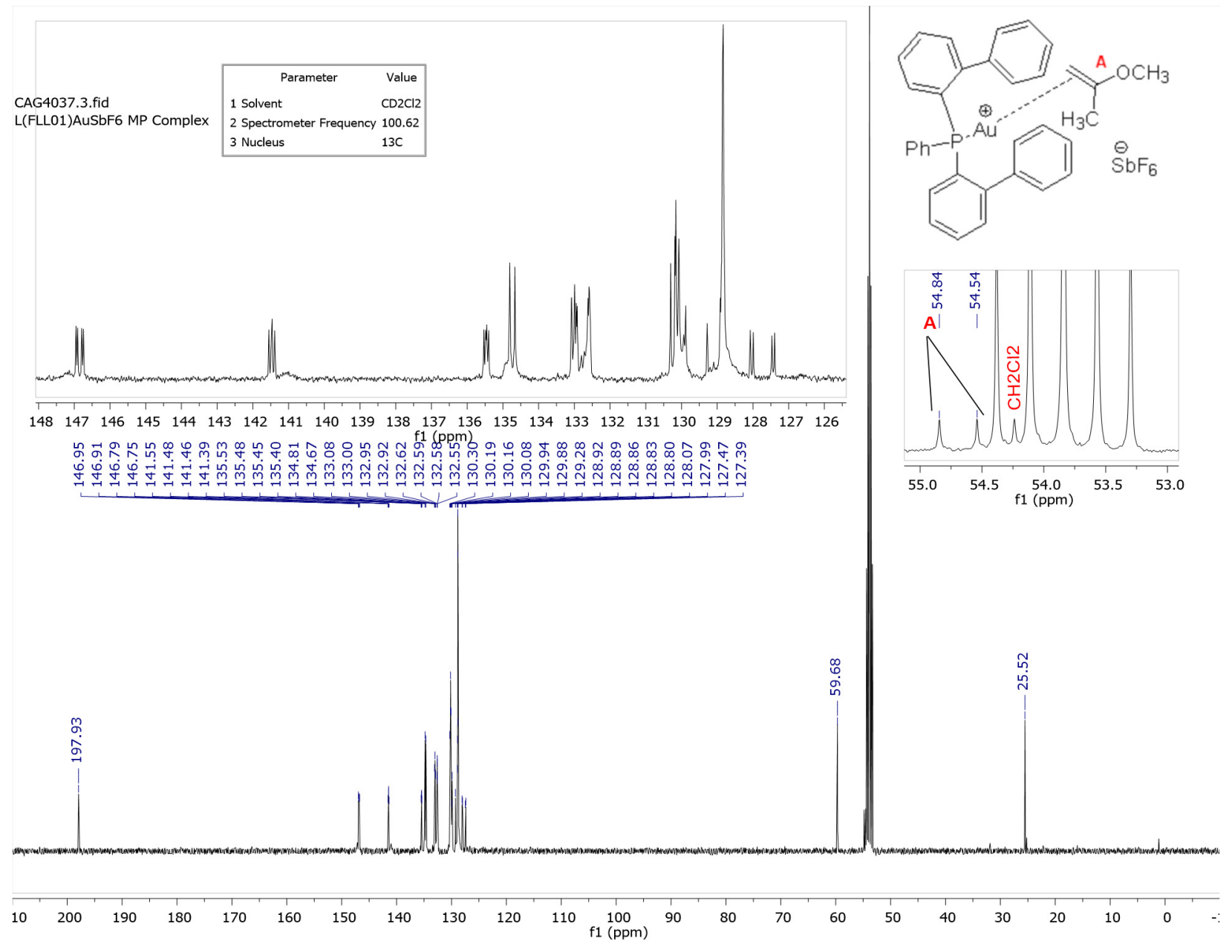

Figure S24. ${ }^{13} \mathrm{C}$ NMR spectrum of $2 \mathrm{c}\left(100.62 \mathrm{MHz}, \mathrm{CD}_{2} \mathrm{Cl}_{2}\right)$. 


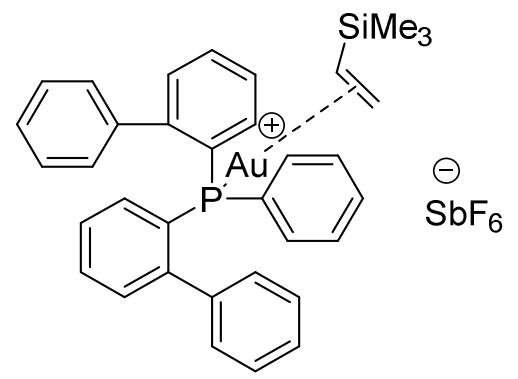

Vinyl trimethylsilane complex (2d).

In a glove box, $\mathrm{AgSbF}_{6}(26 \mathrm{mg}, 0.077 \mathrm{mmol})$ was added to a vial. A separate vial containing $\mathrm{LAuCl}$ (50mg, 0.077 mmol) was vacuumed, then refilled with Ar. To the vial containing $\mathrm{LAuCl}$ was added $3 \mathrm{~mL}$ of anhydrous $\mathrm{CH}_{2} \mathrm{Cl}_{2}$. The mixture was swirled until the gold was dissolved. The dissolved gold mixture was then added to the vial containing the $\mathrm{AgSbF}_{6}$. VinylSiMe 3 (57 $\left.\mu 1,0.385 \mathrm{mmol}\right)$ was then added to the vial. The resulting mixture was sonicated for two minutes, then filtered through celite into a flask and dried under vacuum, affording a white/pink solid. The solid was recrystallized overnight in 1:4 anhydrous $\mathrm{CH}_{2} \mathrm{Cl}_{2}$ :MS-grade hexane. The resulting crystals were dried under vacuum, resulting in a dry, white/pink solid (46.9mg, $64.3 \%$ yield).

${ }^{1} \mathbf{H}$ NMR (400 MHz, $\mathbf{C D}_{2} \mathbf{C l}_{2}$ ): $\delta$ 7.79-7.26 (m, 19H), 6.94 (br d, J=7 Hz, 4H), 5.28 (ddd, J JH=19.8, $\left.13, \mathrm{~J}_{\mathrm{HP}}=2.3 \mathrm{~Hz}, 1 \mathrm{H}\right), 4.43\left(\mathrm{~d} 2, \mathrm{~J}_{\mathrm{HH}}=13,3.4, \mathrm{~J}_{\mathrm{HP}}=3.4 \mathrm{~Hz}, 1 \mathrm{H}\right), 4.31\left(\mathrm{dt}, \mathrm{J}_{\mathrm{HH}}=19.8,3.4, \mathrm{~J}_{\mathrm{HP}}=3.4 \mathrm{~Hz}\right.$, $1 \mathrm{H}), 0.02(\mathrm{~s}, 9 \mathrm{H})$.

${ }^{13}$ C NMR (100 MHz, CD $\left.\mathbf{C l}_{2}\right): \delta 146.84(\mathrm{~d}, \mathrm{~J}=16.1 \mathrm{~Hz}), 146.65$ (d, J=16.1 Hz), 141.35 (d, J=7.6 $\mathrm{Hz}), 141.18$ (d, J=7.8 Hz), 136.35 (d, J=8.1 Hz), 135.83 (d, J=5.7 Hz), 135.69 (d, J=5.8 Hz), 134.36 (d, J=13.8 Hz), [133.34, 133.30, 133.27, 133.26, 133.19, 133.18, 133.15, 133.14],* 130.41 (d, $\mathrm{J}=12.1 \mathrm{~Hz}$ ), 130.16 (s), 129.88 (s), 129.49 (s), 129.36 (s), 129.31 (d, J=8.5 Hz), 129.19 (d, J=8.4 Hz), 129.13 (s), 129.03 (s), 128.77 (d, J=61.8 Hz), 127.20 (d, J=62.5 Hz), 126.50 (d, J=62.7 Hz), $118.15(\mathrm{~d}, \mathrm{~J}=10.3 \mathrm{~Hz}),-1.22(\mathrm{~s}) .{ }^{*}$ Bracketed italics signals are overlapping multiplets that are not clearly resolvable.

${ }^{31}$ P NMR (162 MHz, $\left.\mathrm{CD}_{2} \mathrm{Cl}_{2}\right): \delta 21.6$.

Elemental Analysis: Calcd (Found) for $\mathrm{C}_{35} \mathrm{H}_{34} \mathrm{AuF}_{6} \mathrm{PSbSi}$ : C, 44.42 (44.04), H: 3.62 (3.80). (Corresponding yield, $0.039 \mathrm{~g}, 53.7 \%$ ). 


\begin{tabular}{|lc|}
\hline \multicolumn{1}{|c|}{ Parameter } & Value \\
1 Solvent & $\mathrm{CD2Cl2}$ \\
2 Spectrometer Frequency & 400.17 \\
3 Nucleus & $1 \mathrm{H}$ \\
\hline
\end{tabular}
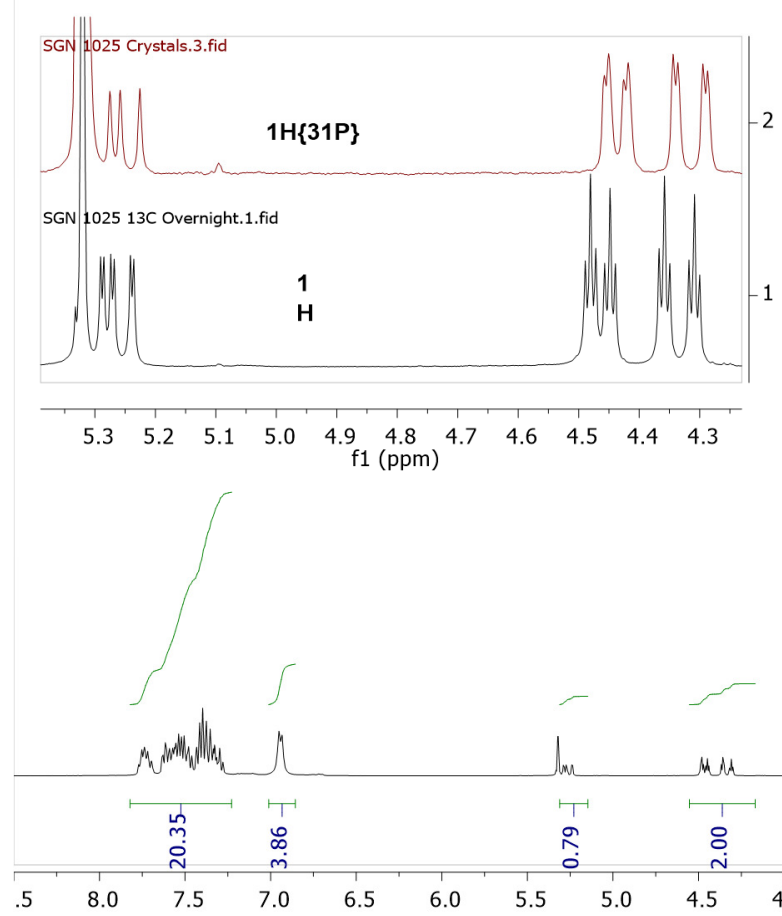

Figure S25. ${ }^{1} \mathrm{H}$ NMR spectrum of $\mathbf{2 d}\left(400.17 \mathrm{MHz}, \mathrm{CD}_{2} \mathrm{Cl}_{2}\right)$.

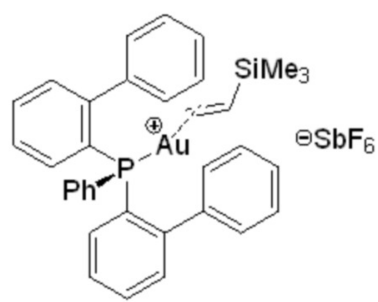

SGN 1025 Crystals.2.fid

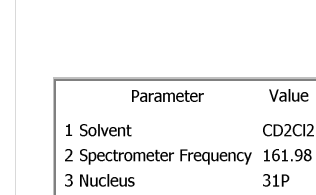

$\stackrel{\text { กิ }}{\text { กิ }}$

2 Spectrometer Frequench $31 \mathrm{P}$

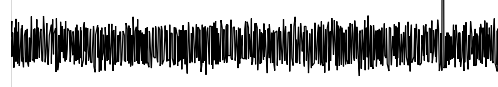

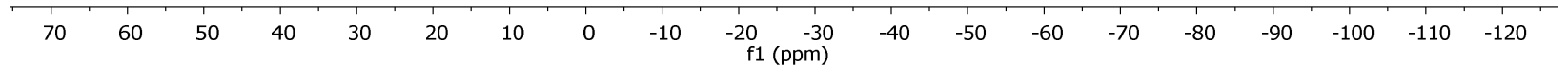

Figure S26. ${ }^{31} \mathrm{P}$ NMR spectrum of $2 d\left(161.98 \mathrm{MHz}, \mathrm{CD}_{2} \mathrm{Cl}_{2}\right)$. 


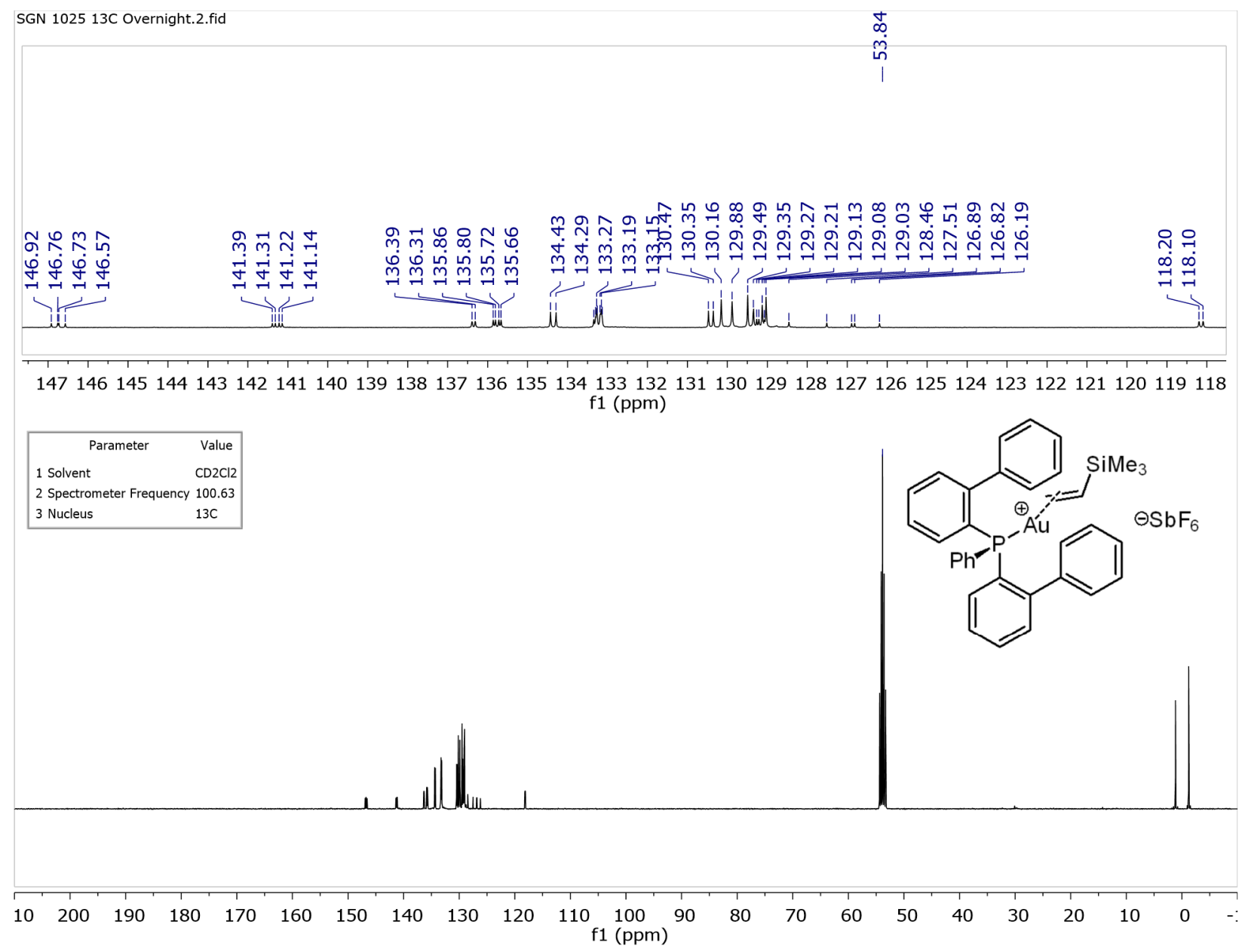

Figure S27. ${ }^{13} \mathrm{C}$ NMR spectrum of $2 \mathbf{d}\left(100.63 \mathrm{MHz}, \mathrm{CD}_{2} \mathrm{Cl}_{2}\right)$. 


\section{Chemistry of di([1,1'-biphenyl]-2-yl)(tert-butyl)phosphane, Ligand 3.}<smiles>CC(C)(C)P(c1ccccc1-c1ccccc1)c1ccccc1-c1ccccc1</smiles>

This compound was prepared previously by a similar method utilizing the analogous Grignard reagent, ${ }^{13}$ C NMR spectroscopic data was not previously reported. ${ }^{2}$

di([1,1'-biphenyl]-2-yl)(tert-butyl)phosphane (3): $n$-Butyllithium $(0.98 \mathrm{~mL}$ of $2.5 \mathrm{M}, 2.5 \mathrm{mmol})$ was added to bromobiphenyl $(0.35 \mathrm{~mL}, 2 \mathrm{mmol})$ at $0^{\circ} \mathrm{C}$ in anyhydrous diethyl ether $(10 \mathrm{ml})$ and stirred for 15 minutes. tert-Butyldichlorophosphine ( $1 \mathrm{~mL}$ of $1 \mathrm{M}$ solution in diethyl ether, $1 \mathrm{mmol}$ ) was added and the solution stirred overnight. The reaction was quenched with $10 \mathrm{~mL}$ of water. The organic and aqueous layers were separated. The organic layer was extracted with $3 \times 20 \mathrm{~mL}$ $\mathrm{CH}_{2} \mathrm{Cl}_{2}$, dried with sodium sulfate and decanted. The solvent was removed in vacuo and the crude product was purified by recrystallization from 1:1 $\mathrm{CH}_{2} \mathrm{Cl}_{2}$ :Hexanes at room temperature to yield a cream colored solid $(0.22 \mathrm{~g}, 56 \%$ yield $)$.

${ }^{1} \mathbf{H}$ NMR (400 MHz, CD2 Cl2): $\delta 7.62\left(\mathrm{dt}, \mathrm{J}_{\mathrm{HH}}=7.4,1.8, \mathrm{~J}_{\mathrm{HP}}=1.8 \mathrm{~Hz}, 2 \mathrm{H}\right), 7.38-7.29(\mathrm{~m}, 4 \mathrm{H})$, 7.28-7.17 (m, 6H), 7.14 (ddd, $\left.\mathrm{J}_{\mathrm{HH}}=7.2,1.8 \mathrm{~Hz}, \mathrm{~J}_{\mathrm{HP}}=4.3 \mathrm{~Hz}, 2 \mathrm{H}\right), 0.90\left(\mathrm{~d}, \mathrm{~J}_{\mathrm{HP}}=12.2 \mathrm{~Hz}, 9 \mathrm{H}\right)$.

${ }^{13}$ C NMR (75 MHz, CD2Cl $\left.\mathbf{C l}_{2}\right): \delta 149.02(\mathrm{~d}, \mathrm{~J}=28.2 \mathrm{~Hz}), 143.3(\mathrm{~d}, \mathrm{~J}=5.0 \mathrm{~Hz}), 136.8(\mathrm{~d}, \mathrm{~J}=25.5$ $\mathrm{Hz}), 135.3$ (d, J = 1.7 Hz), 131.3 (d, J = 4.7 Hz), 131.06 (d, J = 3.8 Hz), 128.6, 127.9, 127.1, 127.0.

${ }^{31} \mathbf{P}\left\{{ }^{1} \mathrm{H}\right\}$ NMR (121.5MHz, $\left.\mathrm{CD}_{2} \mathrm{Cl}_{2}\right): \delta-5.8$

(S2) Stambuli, J. P, Stauffer, S. R., Shaughnessy, K. H.; Hartwig, J. F. J. Am. Chem. Soc. 2001, 123, $2677-2678$. 

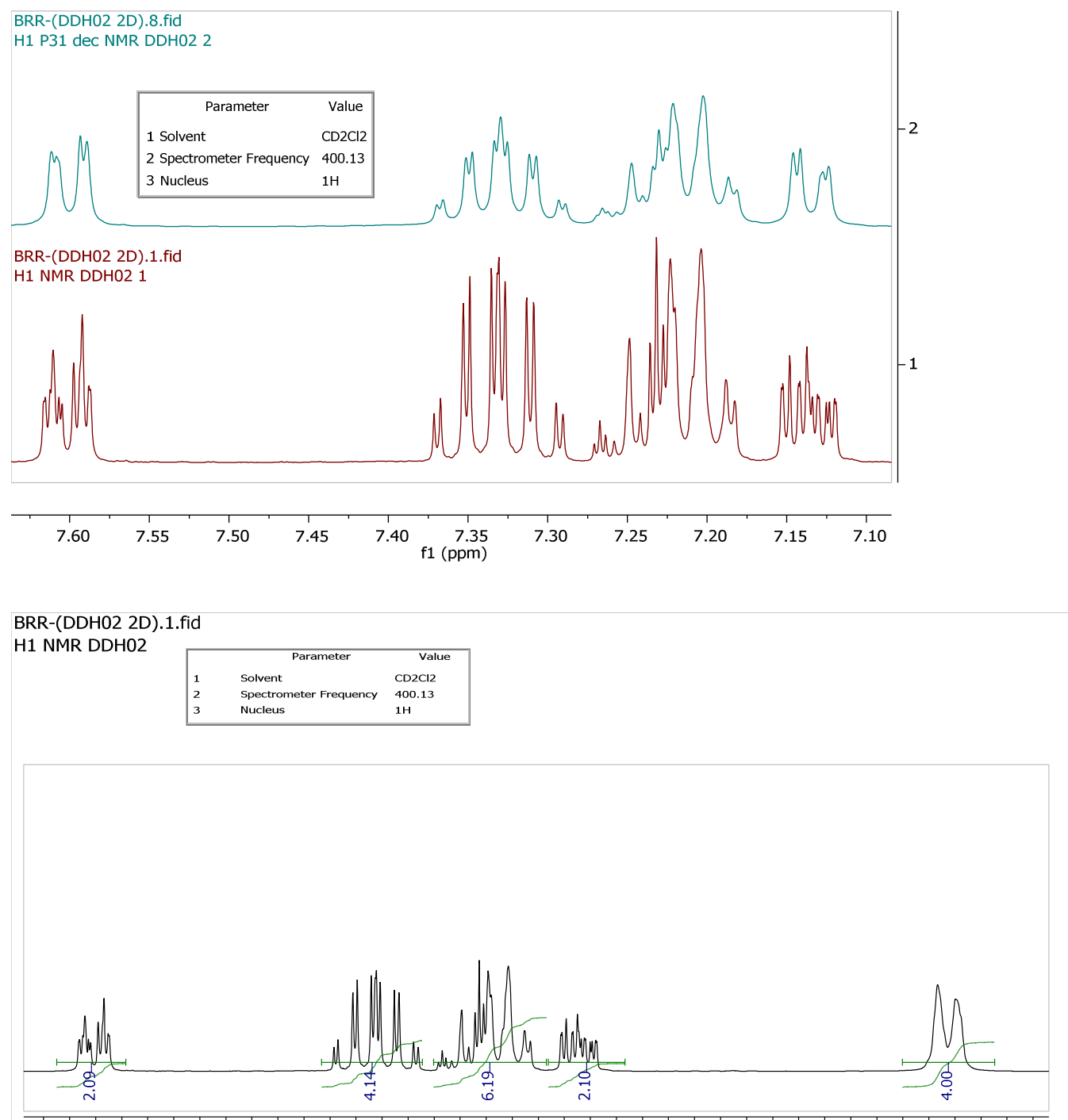

\begin{tabular}{lllllllllllllllllllll}
\hline 7.65 & 7.60 & 7.55 & 7.50 & 7.45 & 7.40 & 7.35 & 7.30 & 7.25 & 7.20 & 7.15 & 7.10 & 7.05 & 7.00 & 6.95 & 6.90 & 6.85 & 6.80 & 6.75 & 6.70
\end{tabular} f1 (ppm)
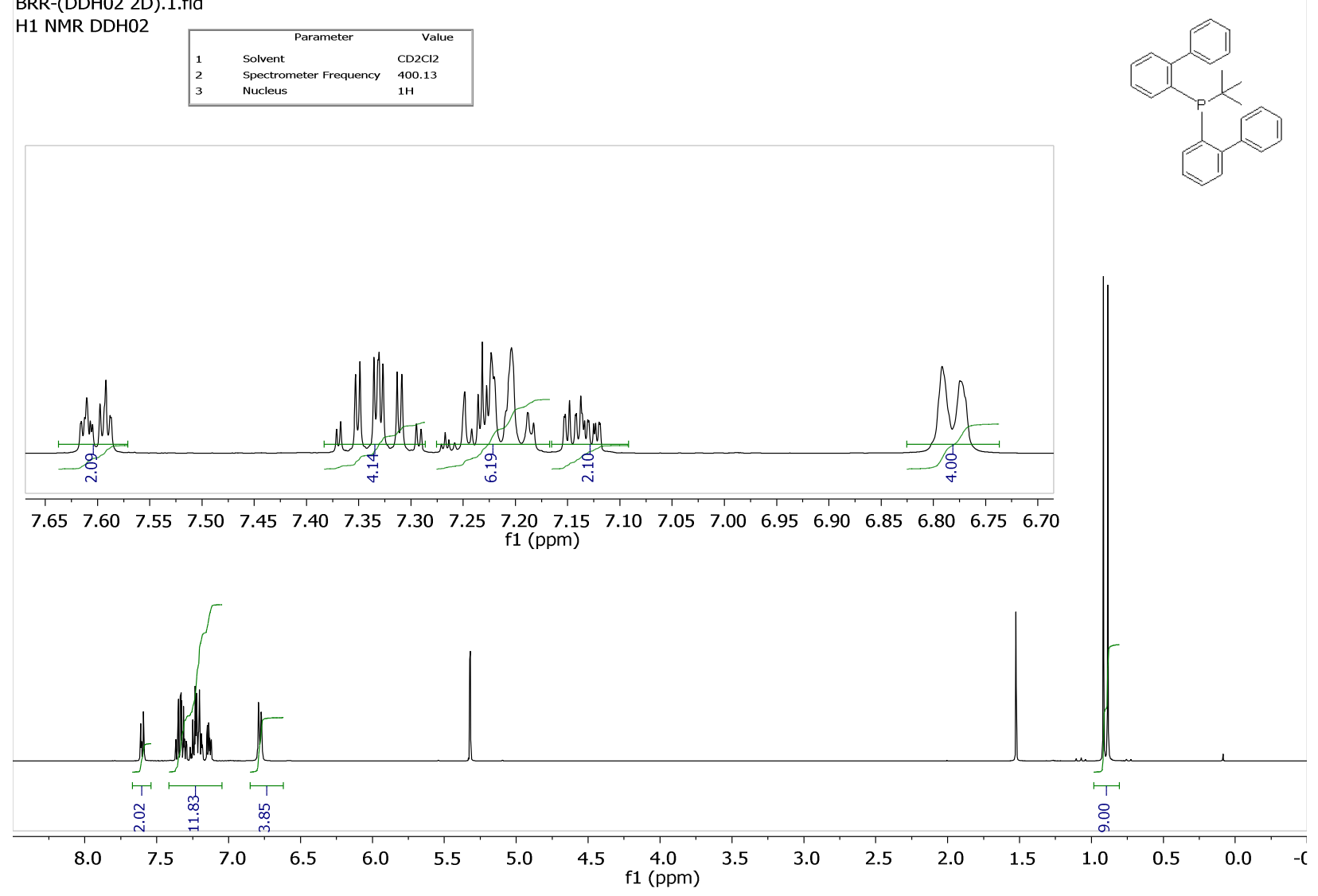

Figure S28. ${ }^{1} \mathrm{H}$ NMR spectra $\left(400.13 \mathrm{MHz}, \mathrm{CD}_{2} \mathrm{Cl}_{2}\right.$ ) of $\mathbf{3}$ with stack plot (above) showing phosphorous decoupled spectra. 


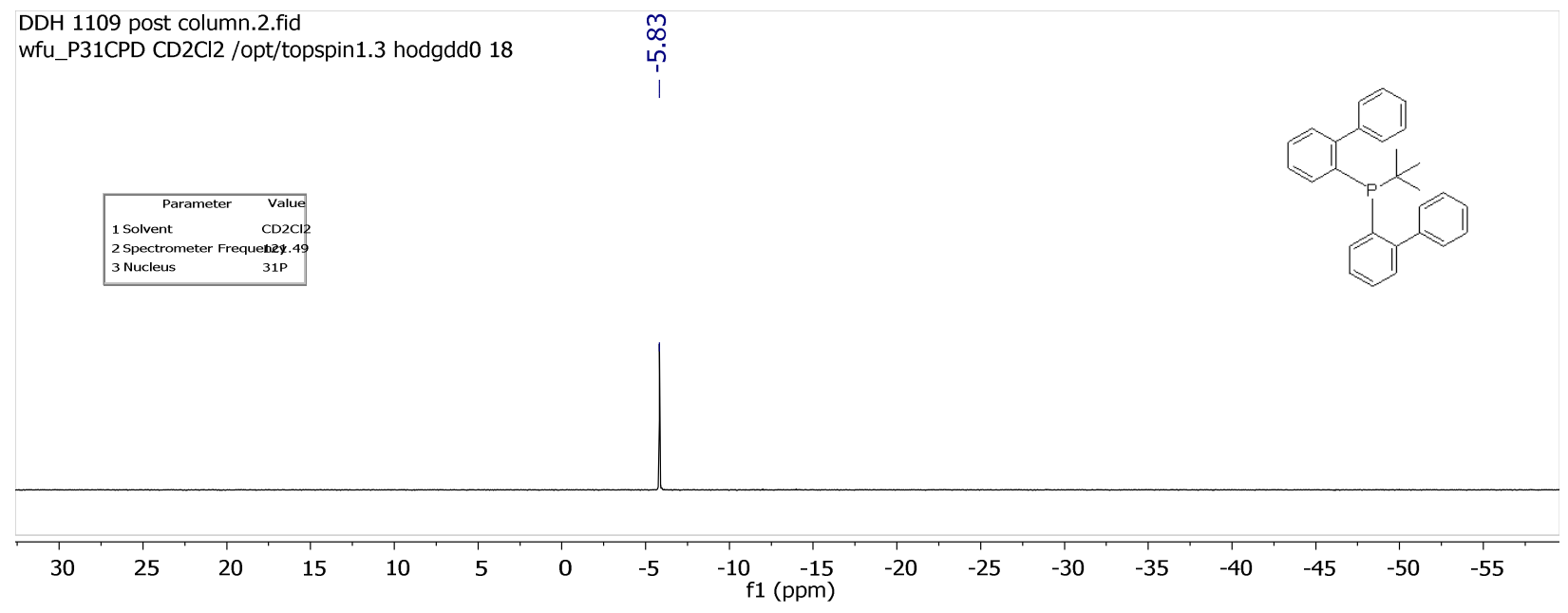

Figure S29. ${ }^{31} \mathrm{P}$ NMR Spectrum of $3\left(121.49 \mathrm{MHz}, \mathrm{CD}_{2} \mathrm{Cl}_{2}\right)$.

DDH 1109 post column.4.fid

wfu_C13CPD_128 CD2C12 /opt/topspin1.3 hodgdd0 24

\begin{tabular}{|lll|}
\hline & \multicolumn{1}{c}{ Parameter } & \multicolumn{1}{c|}{ Value } \\
1 & Solvent & CD2C12 \\
2 & Spectrometer Frequency & 75.48 \\
3 & Nucleus & $13 \mathrm{C}$ \\
\hline
\end{tabular}

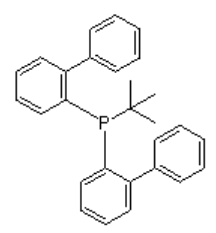

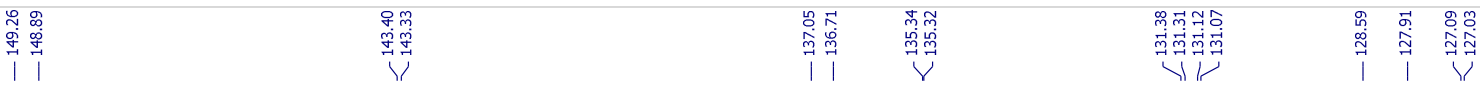
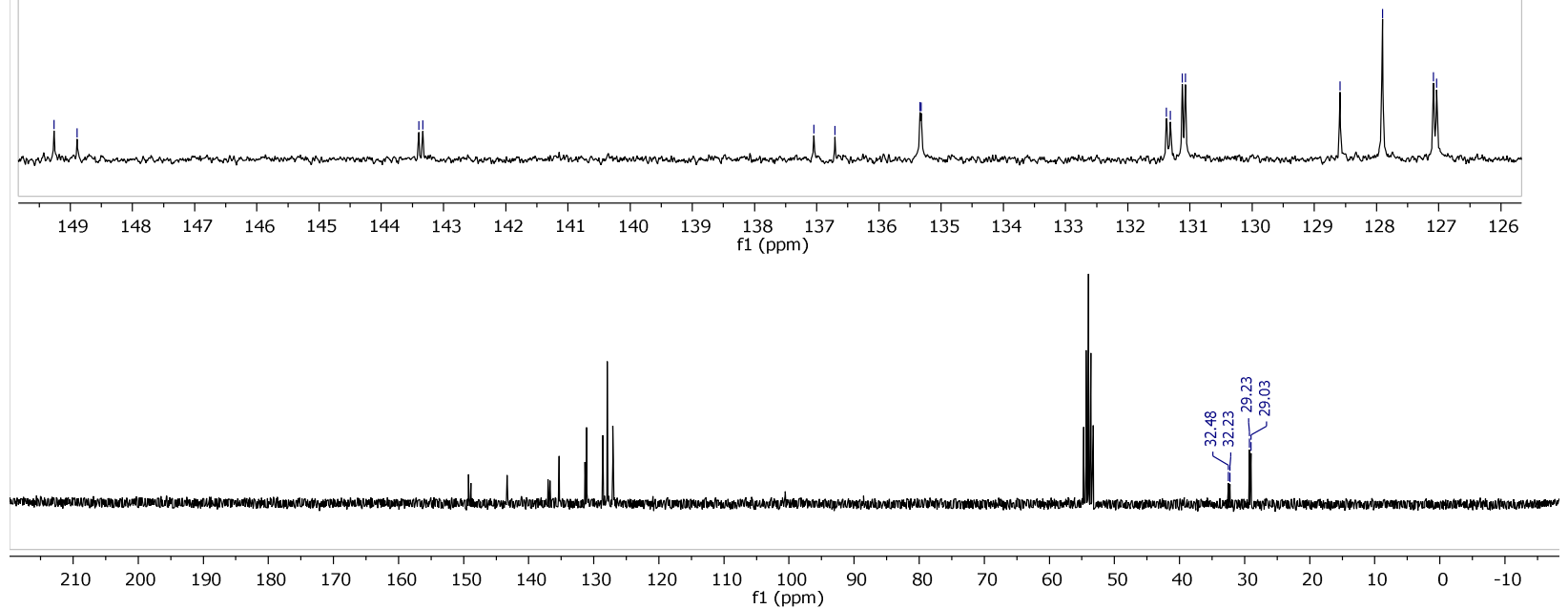

Figure S30. ${ }^{13} \mathrm{C}$ NMR Spectrum of $3\left(75.48 \mathrm{MHz}, \mathrm{CD}_{2} \mathrm{Cl}_{2}\right)$. 


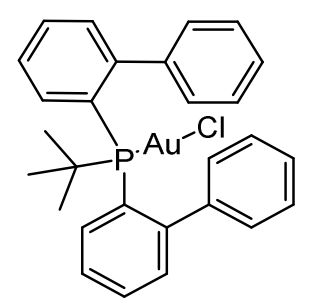

Gold(I) di([1,1'-biphenyl]-2-yl)(tert-butyl)phosphane Chloride (3a): di([1,1'-biphenyl]-2yl)(tert-butyl)phosphane $(0.394 \mathrm{~g}, 0.99 \mathrm{mmol})$ and of dimethylsulfidegold(I) chloride $(0.294 \mathrm{~g}$, $0.99 \mathrm{mmol}$ ) were dissolved in methylene chloride and the phosphane ligand added to the dimethylsulfidegold(I) chloride. After 3 hours the solvent was removed via vacuum. The solid was dissolved in 1:2 methylene chloride/hexane to produced crystals (white solid, $0.52 \mathrm{~g}, 82 \%$ ).

${ }^{1} \mathbf{H}$ NMR (300 MHz, $\left.\mathbf{C D}_{2} \mathbf{C l}_{2}\right): \delta 7.63\left(\mathrm{dddd}, \mathrm{J}_{\mathrm{HP}}=9.8, \mathrm{~J}_{\mathrm{HH}}=7.8,1.4,0.4,2 \mathrm{H}\right), 7.52\left(\mathrm{tt}, \mathrm{J}_{\mathrm{HH}}=7.5\right.$, $\left.1.5 \mathrm{~Hz}, \mathrm{~J}_{\mathrm{HP}}=1.5 \mathrm{~Hz}, 2 \mathrm{H}\right), 7.44\left(\mathrm{tt}, \mathrm{J}_{\mathrm{HH}}=7.5,1.6 \mathrm{~Hz}, \mathrm{~J}_{\mathrm{HP}}=1.6 \mathrm{~Hz}, 2 \mathrm{H}\right), 7.36(\mathrm{br} \mathrm{t}, \mathrm{J}=7.0 \mathrm{~Hz}, 2 \mathrm{H})$, 7.52 (dddd, $\left.\mathrm{J}_{\mathrm{HH}}=7.6,1.5,0.3 \mathrm{~Hz}, \mathrm{~J}_{\mathrm{HP}}=4.6 \mathrm{~Hz}, 2 \mathrm{H}\right), 7.07$ (br s, 2H), 6.09 (br s, 2H), (m, 16H), $6.10(\mathrm{br} \mathrm{s}, 2 \mathrm{H}), 1.28\left(\mathrm{~d}, J_{\mathrm{HP}}=16.5 \mathrm{~Hz}, 9 \mathrm{H}\right)$. One broad hydrogen signal is not clearly detectable but appears to be in the 7.4 ppm range $(4 H)$.

${ }^{13} \mathrm{C}$ NMR (75 MHz, $\left.\mathbf{C D}_{2} \mathbf{C l}_{2}\right): \delta 148.17\left(\mathrm{~d}, \mathrm{~J}_{\mathrm{CP}}=12.6 \mathrm{~Hz}\right), 141.14\left(\mathrm{~d}, \mathrm{~J}_{\mathrm{CP}}=5.3 \mathrm{~Hz}\right) 133.99(\mathrm{~d}$, $\left.\mathrm{J}_{\mathrm{CP}}=5.4 \mathrm{~Hz}\right), 133.3\left(\mathrm{~d}, \mathrm{~J}_{\mathrm{CP}}=8.1 \mathrm{~Hz}\right), 130.85\left(\mathrm{~d}, \mathrm{~J}_{\mathrm{CP}}=2.3 \mathrm{~Hz}\right), 130.64(\mathrm{br} \mathrm{s}), 128.53\left(\mathrm{~d}, \mathrm{~J}_{\mathrm{CP}}=51.9 \mathrm{~Hz}\right)$, $128.59,127.79,127.38\left(\mathrm{~d}, \mathrm{~J}_{\mathrm{CP}}=8.1 \mathrm{~Hz}\right), 35.73\left(\mathrm{~d}, J_{\mathrm{CP}}=32.7 \mathrm{~Hz}\right), 29.19\left(\mathrm{~d}, \mathrm{~J}_{\mathrm{CP}}=7.0 \mathrm{~Hz}\right)$.

\section{${ }^{31} \mathbf{P}\left\{{ }^{1} \mathrm{H}\right\}$ NMR (121.5MHz, $\left.\mathrm{CD}_{2} \mathrm{Cl}_{2}\right): \delta 33.4$}

Elemental Analysis Calcd (found) for $\mathrm{C}_{28} \mathrm{H}_{27} \mathrm{AuClOP}$ : C, 53.64 (53.67); H, 4.19 (4.34).

Corresponding batch yield not recorded. 


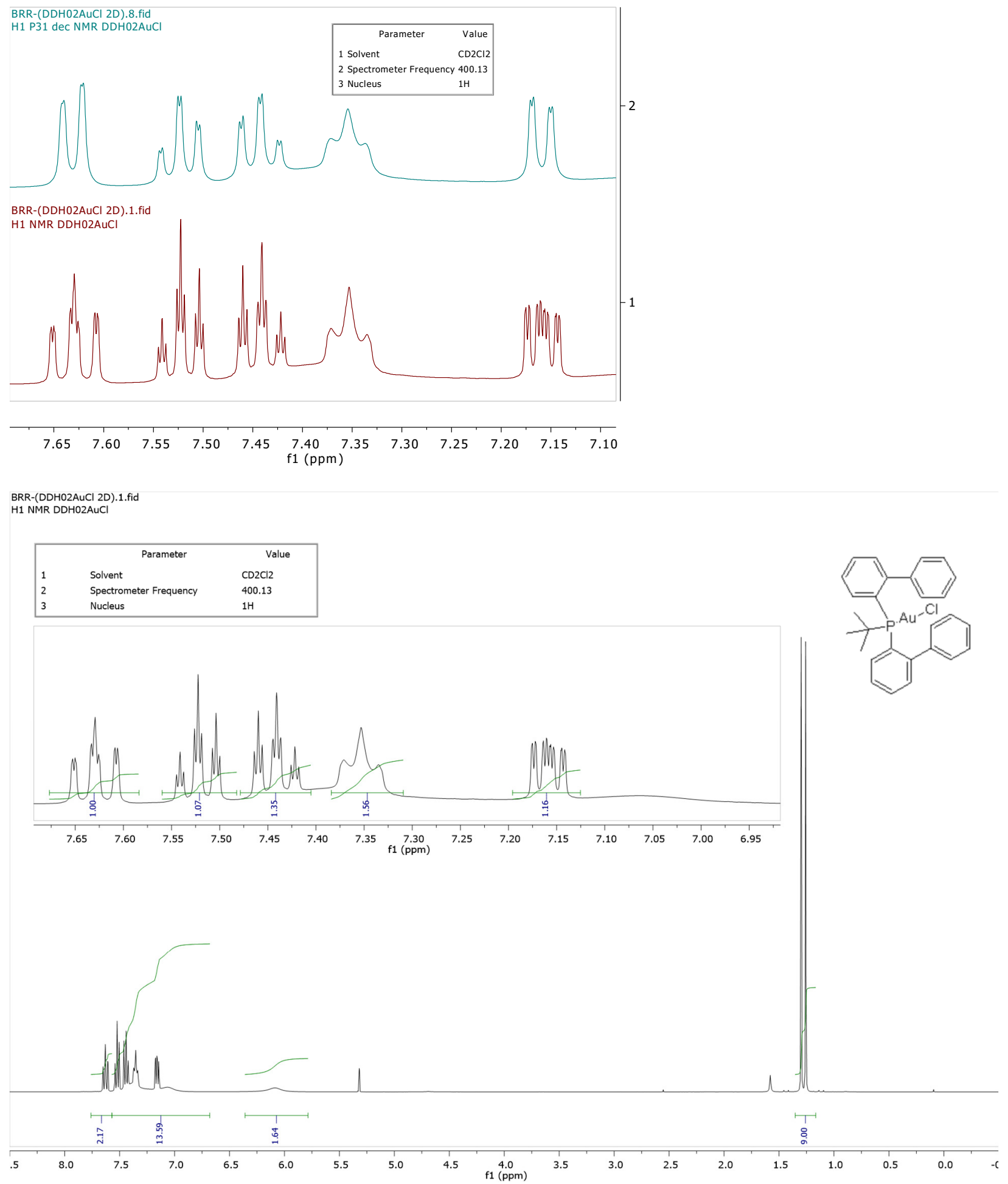

Figure S31. ${ }^{1} \mathrm{H}$ NMR spectra of $3 \mathbf{a}\left(400 \mathrm{MHz}, \mathrm{CD}_{2} \mathrm{Cl}_{2}\right)$ with stack plot (above) showing phosphorous decoupled spectra. 


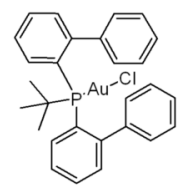

\begin{tabular}{|lll|}
\hline \multicolumn{1}{|c|}{ Parameter } & \multicolumn{1}{c|}{ Value } \\
1 & Solvent & $\mathrm{CD} 2 \mathrm{Cl} 2$ \\
2 & Spectrometer Frequency & 121.51 \\
3 & Nucleus & $31 \mathrm{P}$ \\
\hline
\end{tabular}

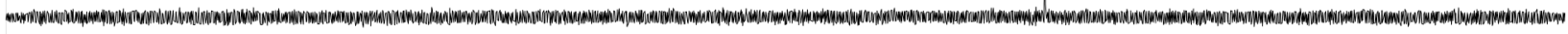

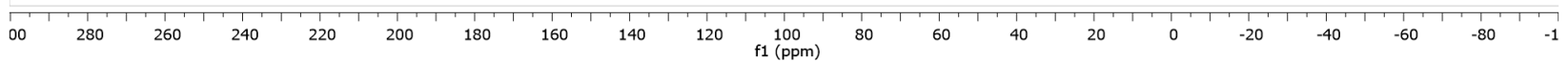

Figure S32. ${ }^{31} \mathrm{P}$ NMR Spectrum of $\mathbf{3 a}\left(121.51 \mathrm{MHz}, \mathrm{CD}_{2} \mathrm{Cl}_{2}\right)$.

DDH 1123 crystals.4.fid

wfu_C13CPD_128 CD2CI2/opt/topspin 1.3 hodgddo 8

\begin{tabular}{|lc|}
\hline \multicolumn{1}{|c|}{ Parameter } & Value \\
1 Solvent & $\mathrm{CD} 2 \mathrm{Cl2}$ \\
2 Spectrometer Frequency & 75.48 \\
3 Nucleus & $13 \mathrm{C}$ \\
\hline
\end{tabular}
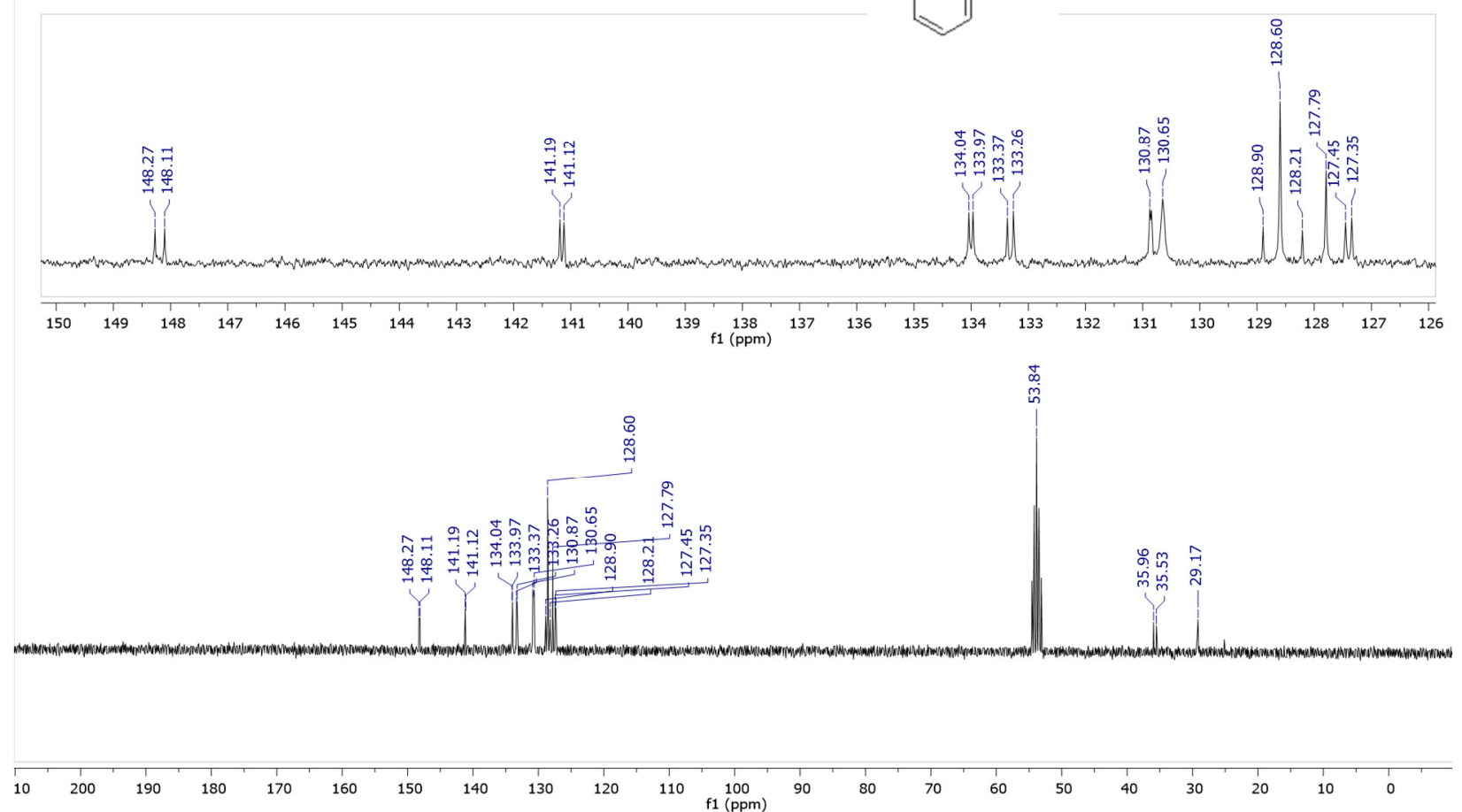

Figure S33. ${ }^{13} \mathrm{C}$ NMR spectrum of $\mathbf{3 a}\left(\mathrm{CD}_{2} \mathrm{Cl}_{2}, 125 \mathrm{MHz}\right)$. 


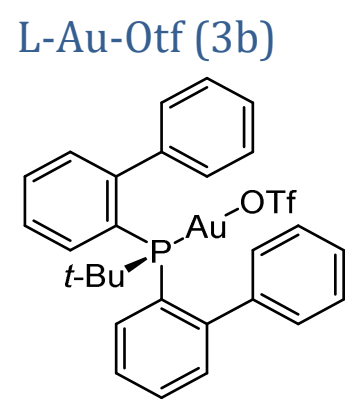

Gold triflate $\mathbf{3 b}$ was prepared according to the same procedure as $\mathbf{1 b}$. The crude product was recrystallized from 1:2 $\mathrm{CH}_{2} \mathrm{Cl}_{2} / \mathrm{Hexanes}$ to give pure product $(0.14 \mathrm{~g}, 92 \%)$.

${ }^{1}$ H NMR Spectrum (400 MHz, CDCl3): $7.61(\mathrm{dd}, \mathrm{J}=8.6,7.8,2 \mathrm{H}), 7.55$ (tt, J=7.7, 1.2, 2H), 7.46 (tt, J=7.7, 1.5, 2H), 7.4 (br t, J=6.9 Hz, 2H), 7.19 (ddd, J = 7.6, 4.7, 1.5 Hz, 2H), 7.08 (br triplet, $\mathrm{J} \sim 6.5 \mathrm{~Hz}, 2 \mathrm{H}), 6.00$ (br d, J 4.2 Hz, 2H), 1.28 (d, J = 17.2 Hz, 8H). One $2 \mathrm{H}$ broad signal is obscured in the 7.4 to $7.5 \mathrm{ppm}$ region.

${ }^{13}$ C NMR Spectrum (101 MHz, CDCl3): $\delta 148.0$ (d, J=11.8 Hz), 140.6 (d, J=5.7 Hz), 133.3 (d, $\mathrm{J}=3.4 \mathrm{~Hz}$ ), 133.2 (d, J=1 Hz), 131.2 (d, J=2.3 Hz), 130.2, 129.6, 129.0, 128.3, 128.1, 127.3 (d, $\mathrm{J}=8.8 \mathrm{~Hz}), 126.3$ (d, J=58.0 Hz), 120.3 (q, J $\left.\mathrm{CF}_{\mathrm{C}}=319 \mathrm{~Hz}\right), 35.7$ (d, J=35.6 Hz), 29.1 (d, J = 6.6 Hz).

${ }^{31}$ P NMR Spectrum (162 MHz, CDCl3): $\delta 25.6 \mathrm{ppm}$.

Elemental Analysis Calcd (found) for $\mathrm{C}_{29} \mathrm{H}_{27} \mathrm{AuF}_{3} \mathrm{O}_{3} \mathrm{PS}$ : C, 47.04 (46.44); H, 3.68 (3.84). Although these results are outside the range viewed as establishing analytical purity, they are provided to illustrate the best values obtained to date. Corresponding batch yield not recorded. 


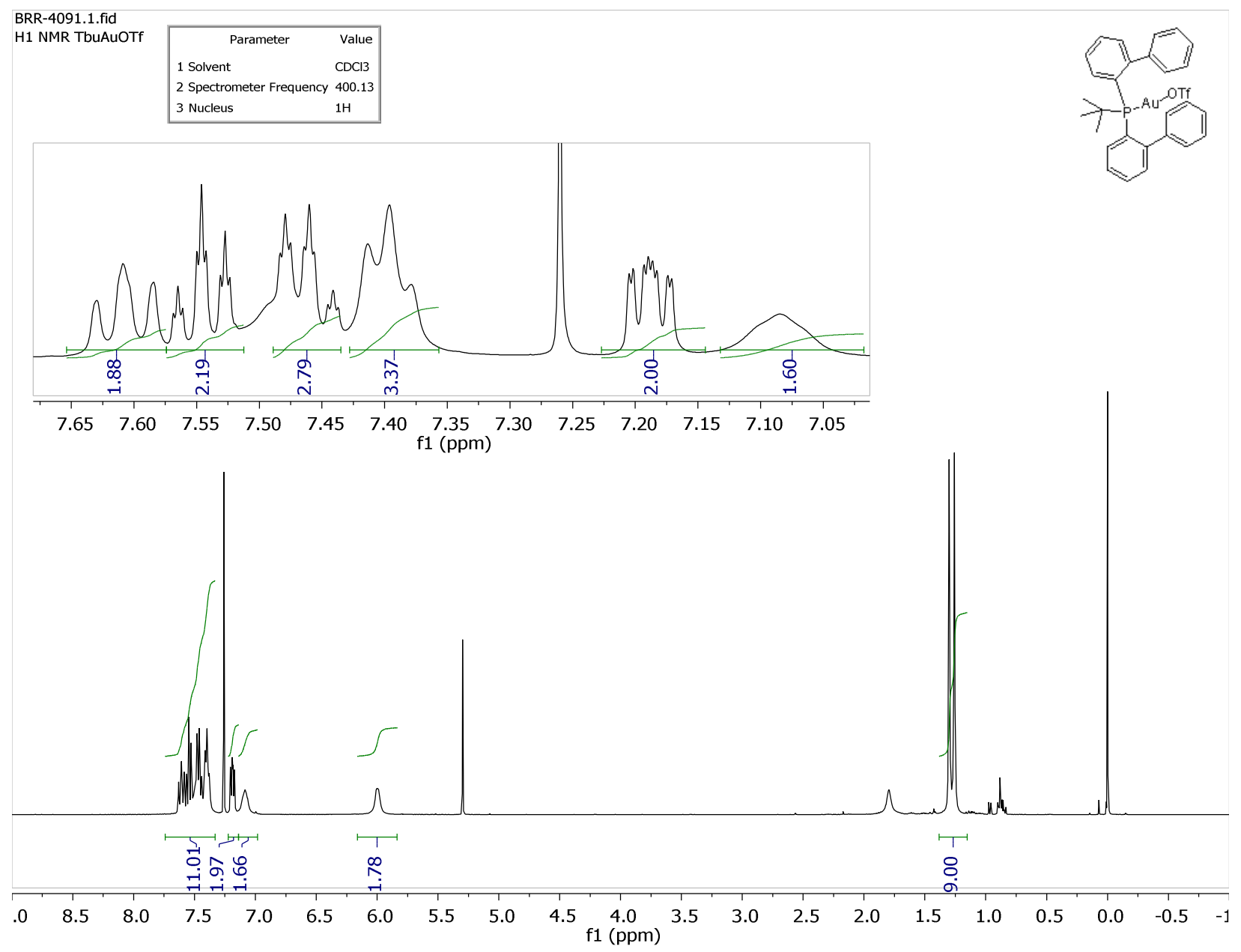

Figure S34. ${ }^{1} \mathrm{H}$ NMR Spectrum of $\mathbf{3 b}\left(400.13 \mathrm{MHz}, \mathrm{CDCl}_{3}\right)$.

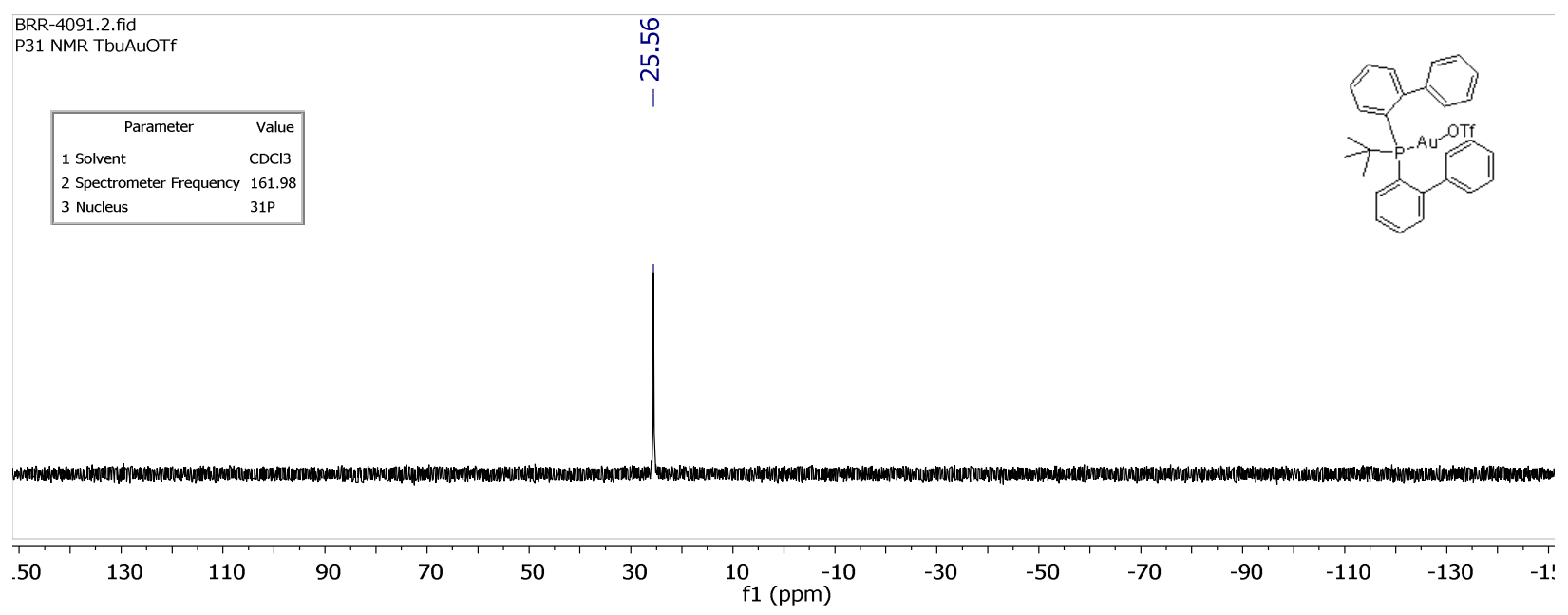

Figure S35. ${ }^{31} \mathrm{P}$ NMR spectrum of $\mathbf{3 b}\left(161.98 \mathrm{MHz}, \mathrm{CDCl}_{3}\right)$. 


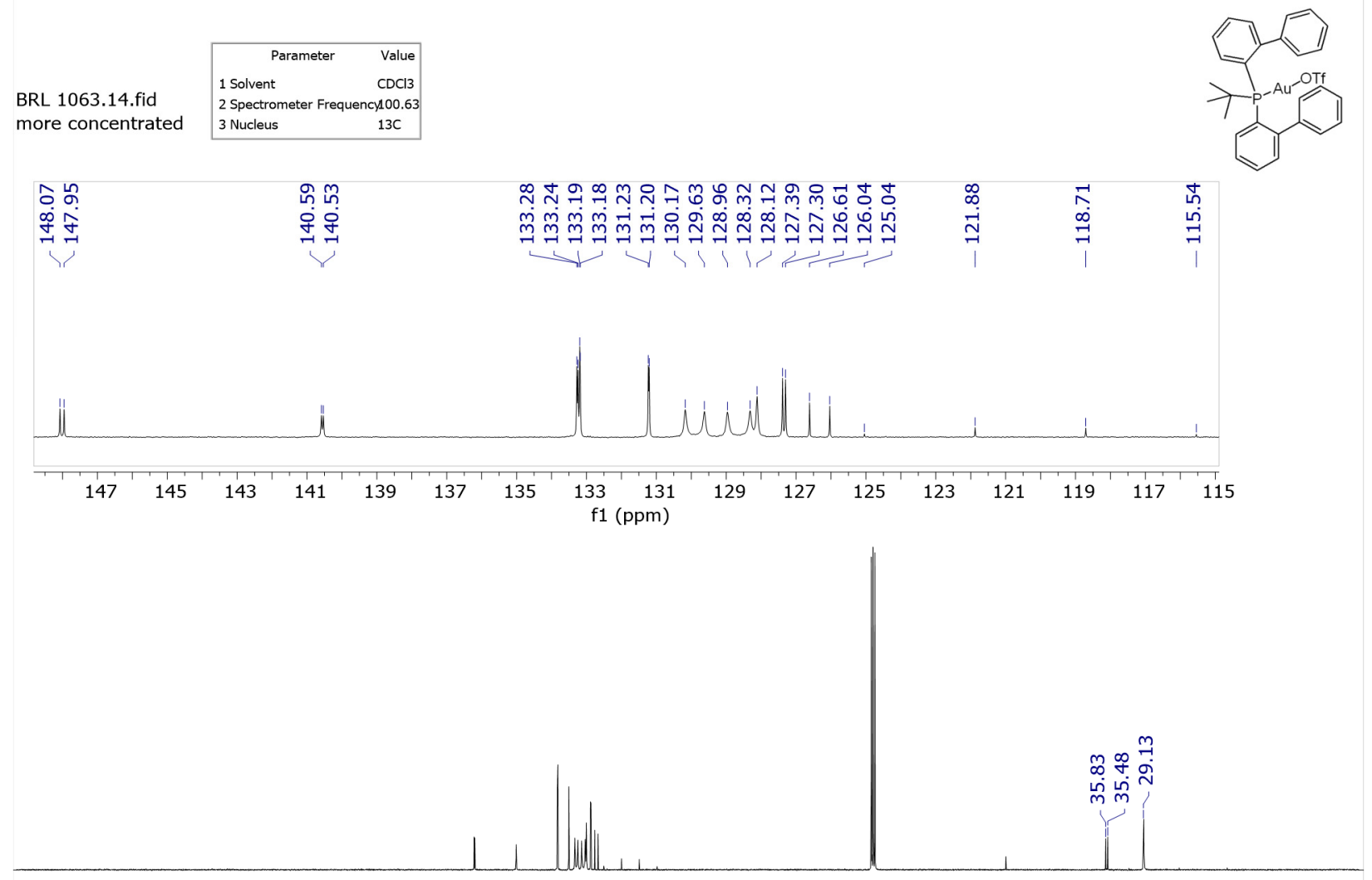

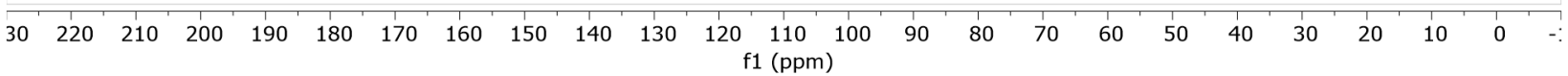

Figure S36. ${ }^{13} \mathrm{C}$ NMR Spectrum of $3 \mathbf{b}\left(100.63 \mathrm{MHz}, \mathrm{CDCl}_{3}\right)$. 


\section{Methoxypropene complex (3c)}

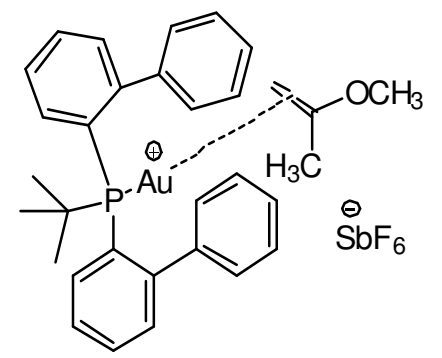

[(t-Bu)(o-biphenyl $\left.)_{2} \mathbf{P A u}\right][$ methoxypropene]SbF6 (3c): $\mathrm{L}-\mathrm{Au}-\mathrm{Cl}(0.05 \mathrm{~g}, 0.077 \mathrm{mmol})$ and $\mathrm{AgSbF}_{6}(0.0265 \mathrm{~g}, 0.077 \mathrm{mmol})$ was dissolved in $1 \mathrm{~mL}$ of anhydrous dichloromethane. The solution was filtered through celite. Methoxypropene $(0.011 \mathrm{~mL}, 0.115 \mathrm{mmol}, 1.5$ equivalents $)$ was added and the solutions was filtered again through a fresh pad of celite. The solvents were removed under reduced pressure to yield a colorless solid ( $0.0298 \mathrm{~g}, 43 \%$ yield $)$.

1H NMR (300 MHz, CD2Cl2): $\delta 7.8(\mathrm{tm}, \mathrm{J}=8.5 \mathrm{~Hz}, 1 \mathrm{H}), 7.71(\mathrm{tm}, \mathrm{J}=8.5), 7.66-7.42(\mathrm{~m}, 8 \mathrm{H})$, 7.36 (br s, 2H), 7.28-7.13 (m, 4H), 6.29 (br t, J=7.7 Hz), 3.61 (s, 3H), 3.08 (dd, J=6.6, $1.6 \mathrm{~Hz}$, 1H), 2.73 (br d, J=6.4 Hz), 2.19 (s, 3H), 1.29 (d, J=17.3 Hz, 9H).

\section{C NMR (126 $\mathrm{MHz}, \mathrm{CD}_{2} \mathrm{Cl}_{2}$, Peaks in bold are coordinated methoxypropene): 194.4,} 147.59 (d, J=14.3 Hz), 147.48 (d, J=14.3 Hz), 142.34 (d, J = 5.9 Hz), 142.25 (d, J = 6.0 Hz), $134.71(\mathrm{~d}, \mathrm{~J}=4.5 \mathrm{~Hz}), 134.40(\mathrm{~d}, \mathrm{~J}=4.6 \mathrm{~Hz}), 133.80(\mathrm{~d}, \mathrm{~J}=8.1 \mathrm{~Hz}), 133.67$ (d, J = 8.2 Hz), $131.92(\mathrm{~d}, \mathrm{~J}=2.6 \mathrm{~Hz}), 131.88(\mathrm{~d}, \mathrm{~J}=2.4 \mathrm{~Hz}), 130.58$, 130.5, 130.22, 129.27, 129.16, 129.07, 128.97, 128.58, 128.35, 128.44 (d, J=7.9 Hz), 128.4 (d, J=8.0 Hz), 126.78 (d, J=50.8 Hz), 126.74 (d, J=51 Hz), 59.25, 55.05 (d, J = 27.7 Hz), 36.02 (d, J = 30.7 Hz), 29.43 (d, J = 7.1 Hz), 25.49. Signals.

31P $\{1$ H $\}$ NMR (121.5 MzH) $\delta 37.2$.

Elemental Analysis Calcd (found) for $\mathrm{C}_{32} \mathrm{H}_{35} \mathrm{AuF}_{6} \mathrm{OPSb}$ : C, 42.74 (42.86); H, 3.92 (3.78). (Corresponding yield, $0.062 \mathrm{~g}, 82.5 \%$ ). 
CAG1127.3.fid

after recryst.

purity check

wfu_PROTON CD2Cl2 /opt/topspin griec12 36

\begin{tabular}{|lr|}
\hline \multicolumn{1}{|c}{ Parameter } & Value \\
1 Solvent & $\mathrm{CD} 2 \mathrm{Cl} 2$ \\
2 Spectrometer Frequency & 300.13 \\
3 Nucleus & $1 \mathrm{H}$ \\
\hline
\end{tabular}
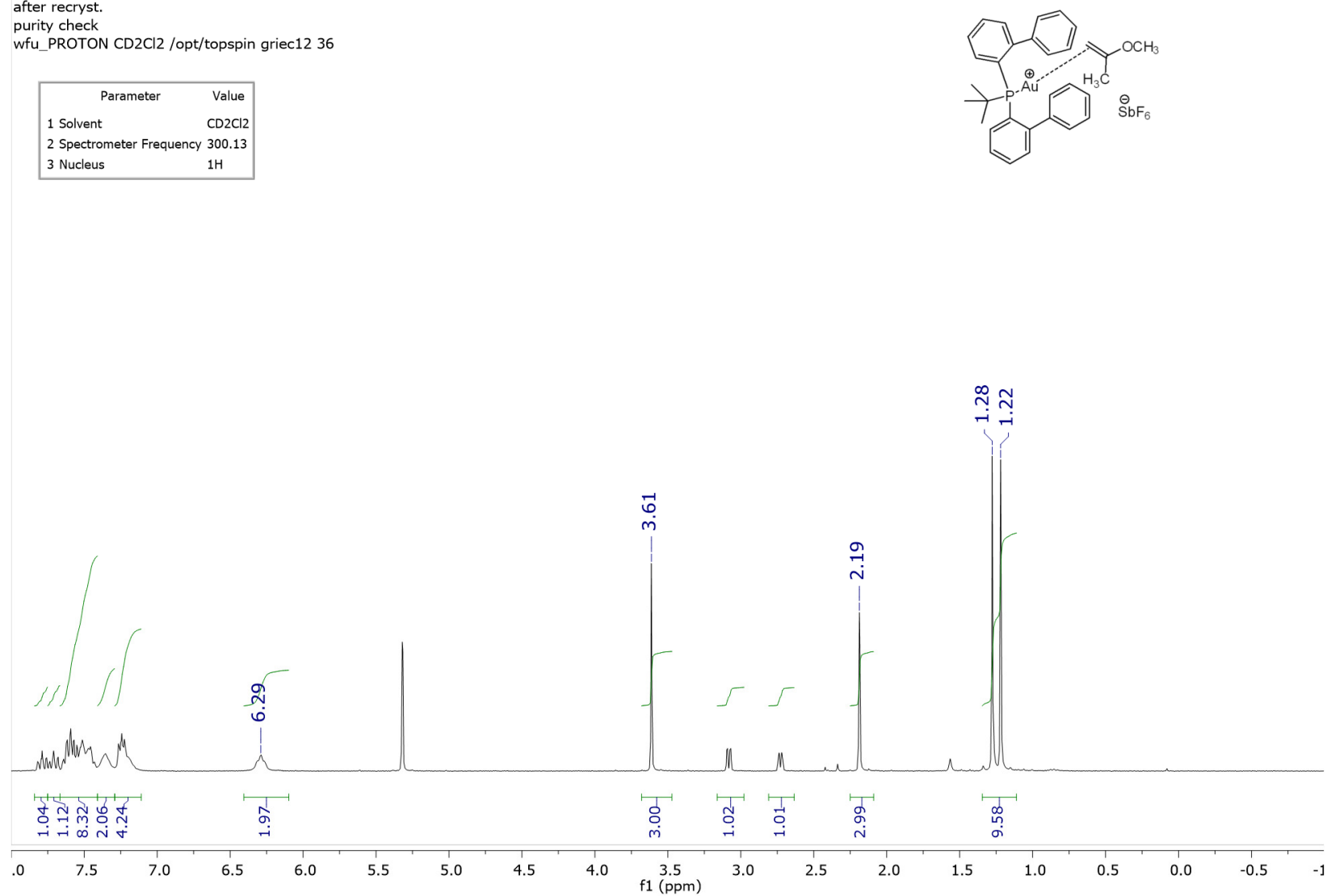

Figure S37. ${ }^{1} \mathrm{H}$ NMR spectrum of $3 \mathbf{c}\left(300.13 \mathrm{MHz}, \mathrm{CD}_{2} \mathrm{Cl}_{2}\right)$.

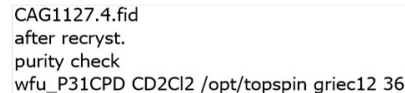

wfu P31CPD $\mathrm{CD} 2 \mathrm{Cl} 2$ /opt/topspin griec 1236

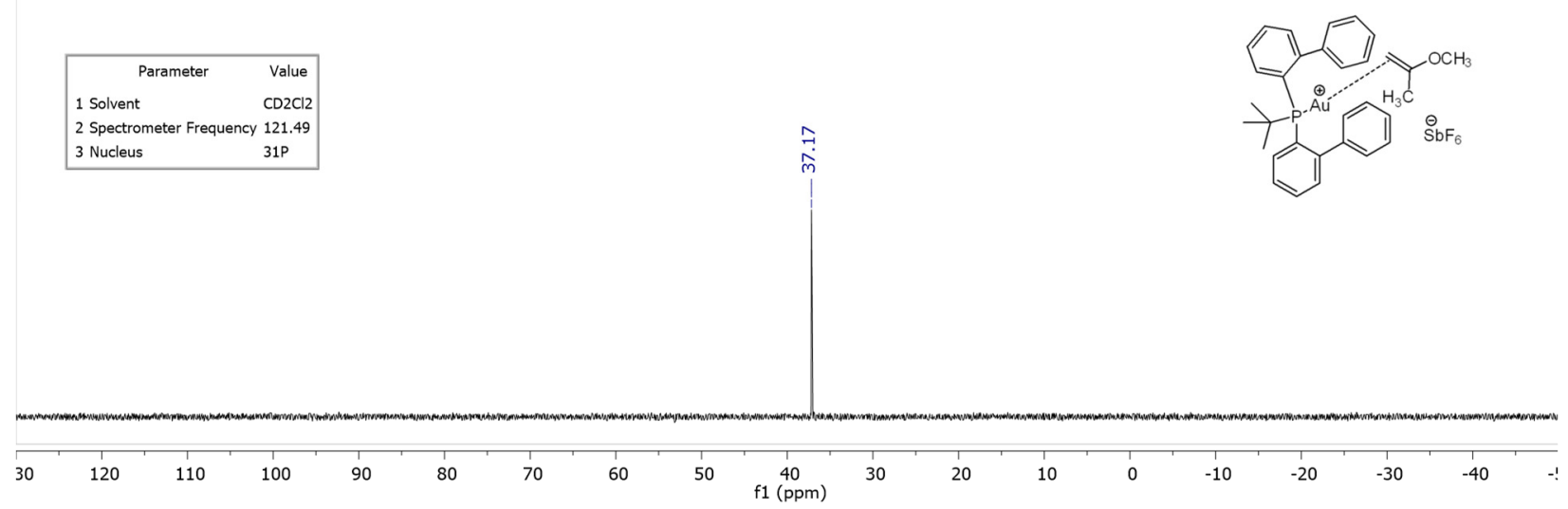

Figure S38. ${ }^{31} \mathrm{P}$ NMR spectrum of $3 \mathbf{c}\left(121.49 \mathrm{MHz}, \mathrm{CD}_{2} \mathrm{Cl}_{2}\right)$. 


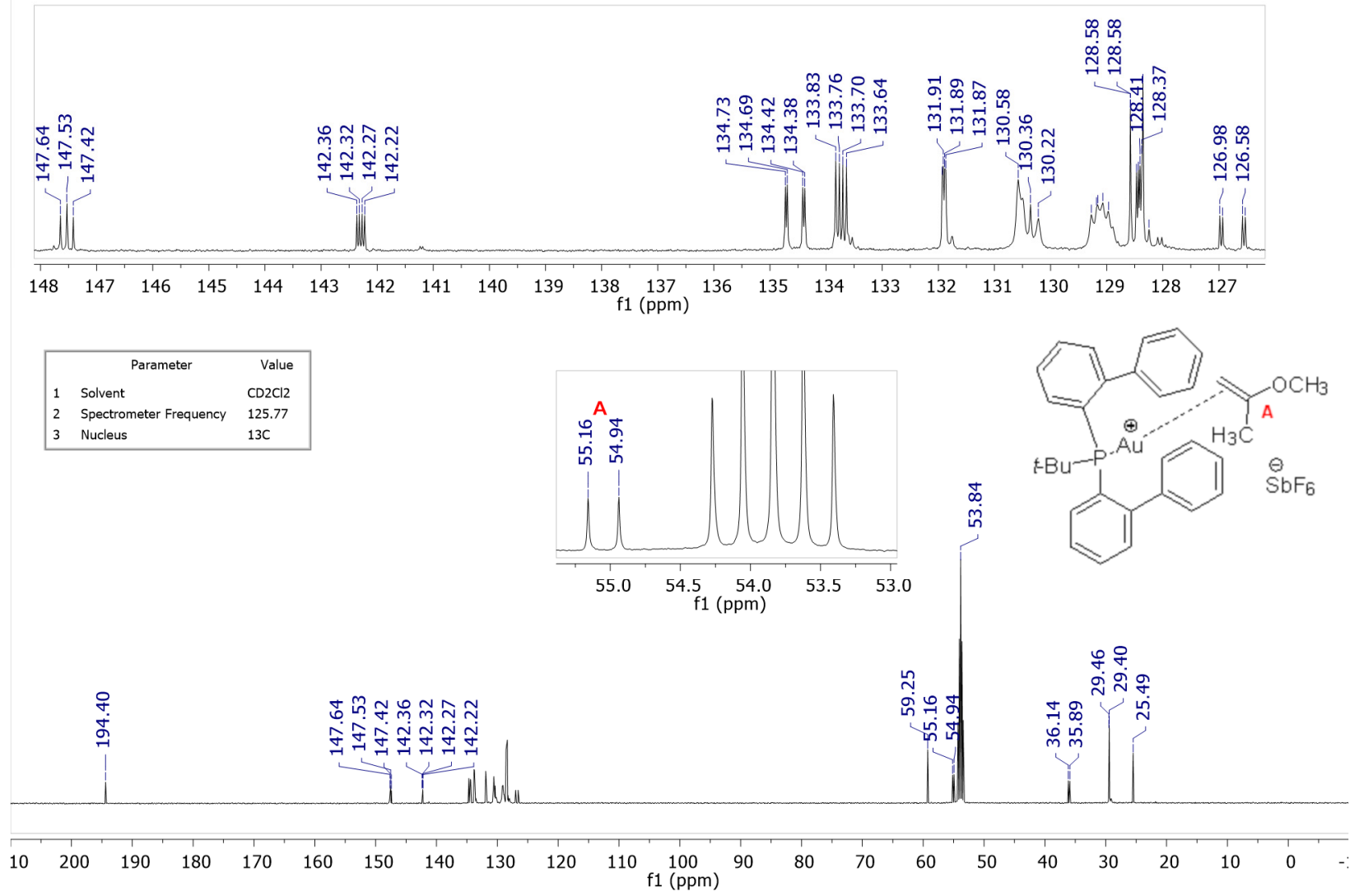

Figure S39. ${ }^{13} \mathrm{C}$ NMR spectrum of $3 \mathbf{c}\left(125.77 \mathrm{MHz}, \mathrm{CD}_{2} \mathrm{Cl}_{2}\right)$, additional minor peaks are from hydrolysis of the enol ether. 


\section{Chemistry of Mono-Biphenyl Complexes}

\section{$\left[\mathrm{Ph}_{2} \mathrm{P}\right.$ (o-biphenyl)Au(Vinyltrimethylsilane) $] \mathrm{SbF}_{6}$ (5d)}

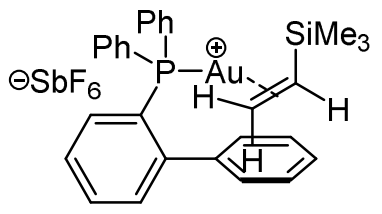

Diphenyl Biphenyl Gold Chloride (0.0078 g, $0.056 \mathrm{mmol})$ and $\mathrm{AgSbF}_{6}(0.0047 \mathrm{~g}, 0.056 \mathrm{mmol})$ were dissolved in $\mathrm{CD}_{2} \mathrm{Cl}_{2}(0.7 \mathrm{~mL})$ and treated with vinyl trimethylsilane $(0.0082 \mathrm{~mL}, 0.0056 \mathrm{~g}, 0.056 \mathrm{mmol})$. Low-temperature NMR spectra were obtained without removal of the silver chloride precipitate. After spectra collection, the solution was decanted and spectra were collected at room temperature the next day. Crystals for solid state analysis could be obtained by diluting pure samples with hexanes (twice the volume of $\mathrm{CH}_{2} \mathrm{Cl}_{2}$ ) and storage at $-20{ }^{\circ} \mathrm{C}$.

${ }^{1} \mathbf{H}$ NMR $\left(\mathbf{C D}_{2} \mathbf{C l}_{2}, \mathbf{5 0 0} \mathbf{M H z},-\mathbf{6 0}^{\circ} \mathbf{C}\right): \delta 7.75-7.37(\mathrm{~m}, 14 \mathrm{H}), 7.3(\mathrm{dd}, J=17.8,13.6,2 \mathrm{H}), 7.03(\mathrm{~d}, J=8.1 \mathrm{~Hz}, 1 \mathrm{H})$, $6.02(\mathrm{ddd}, J=20,12.9,2.4 \mathrm{~Hz}, 1 \mathrm{H}), 5.17(\mathrm{dt}, J=12.9,3.5 \mathrm{~Hz}, 1 \mathrm{H}), 4.93(\mathrm{dt}, J=20,3.6 \mathrm{~Hz}, 1 \mathrm{H}), 0.01$ (s, $9 \mathrm{H})$.

${ }^{13} \mathrm{C}$ NMR $\left(\mathrm{CD}_{2} \mathrm{Cl}_{2}, 125.77 \mathrm{MHz},-60{ }^{\circ} \mathrm{C}\right.$, Peaks in Bold are coordinated Vinyl Trimethylsilane): $\delta 146.45(\mathrm{~d}, \mathrm{~J}=$ $15.6 \mathrm{~Hz}), 140.18$ (d, J = 7.9 Hz), 136.82 (d, J=8.3 Hz), 135.07 (d, J=5.6 Hz), 133.64 (d, J = 13.7 Hz), 133.26 (d, J = $13.7 \mathrm{~Hz}), 132.75$ (d, J=2.5 Hz), 132.62 (d, J=2.3 Hz), 132.53 (d, J=2.2 Hz), 132.15 (d, J = 8.8 Hz), 129.77 (d, J = 11.4 $\mathrm{Hz}$ ), 129.67 (d, J=11.95 Hz), 129.58, 129.52, 128.97, 128.8, 128.75, 128.30 (d, J = 9.4 Hz), 127.05 (d, J = 62.6 Hz), $126.45(\mathrm{~d}, \mathrm{~J}=62.8 \mathrm{~Hz}), 124.21(\mathrm{~d}, \mathrm{~J}=62.5 \mathrm{~Hz}), \mathbf{1 1 9 . 1 7}(\mathbf{d}, \mathbf{J}=\mathbf{9 . 2} \mathbf{H z}), \mathbf{- 2 . 0 5}$.

${ }^{31} \mathrm{P}$ NMR $\left(\mathrm{CD}_{2} \mathrm{Cl}_{2}, 202 \mathrm{MHz},-60{ }^{\circ} \mathrm{C}\right): \delta 30.2$. 
ZWT-8/2

ZWT-8 vinylsilane biphenyl-diphenyl-P-Au -73 oC $1 \mathrm{H}$

\begin{tabular}{|ll|}
\hline \multicolumn{1}{|c|}{ Parameter } & \multicolumn{1}{c|}{ Value } \\
1 Solvent & $\mathrm{CD} 2 \mathrm{Cl} 2$ \\
2 Temperature & 213.2 \\
3 Spectrometer Frequency & 500.13 \\
4 Nucleus & $1 \mathrm{H}$ \\
\hline
\end{tabular}
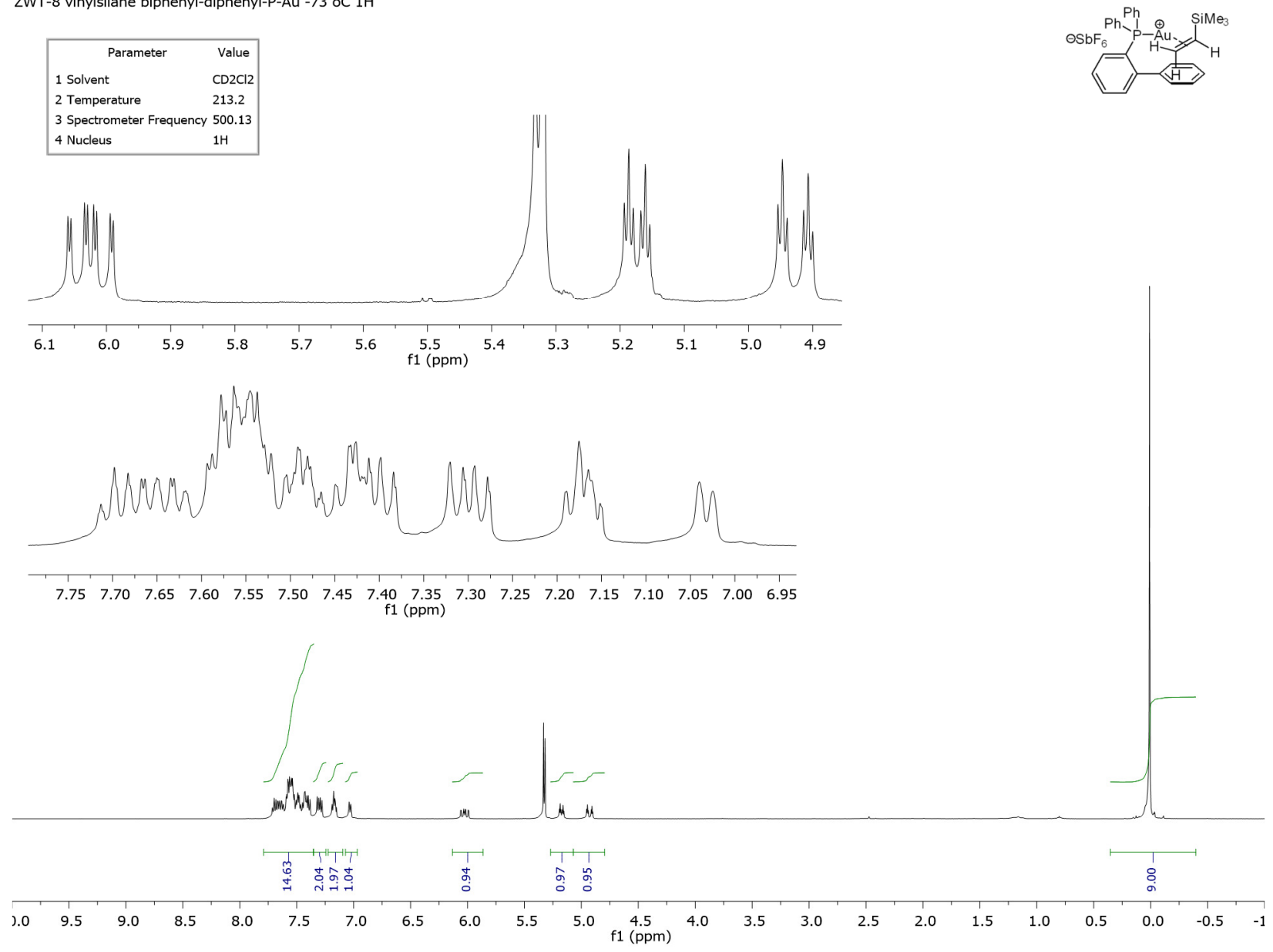

Figure S40. ${ }^{1} \mathrm{H}$ NMR Spectrum of $\mathbf{5 d}\left(500.13 \mathrm{MHz}, \mathrm{CD}_{2} \mathrm{Cl}_{2},-60{ }^{\circ} \mathrm{C}\right)$.

ZWT-8/3
ZWT-8 vinylsilane biphenyl-diphenyl-P-Au -73 oc 31P-

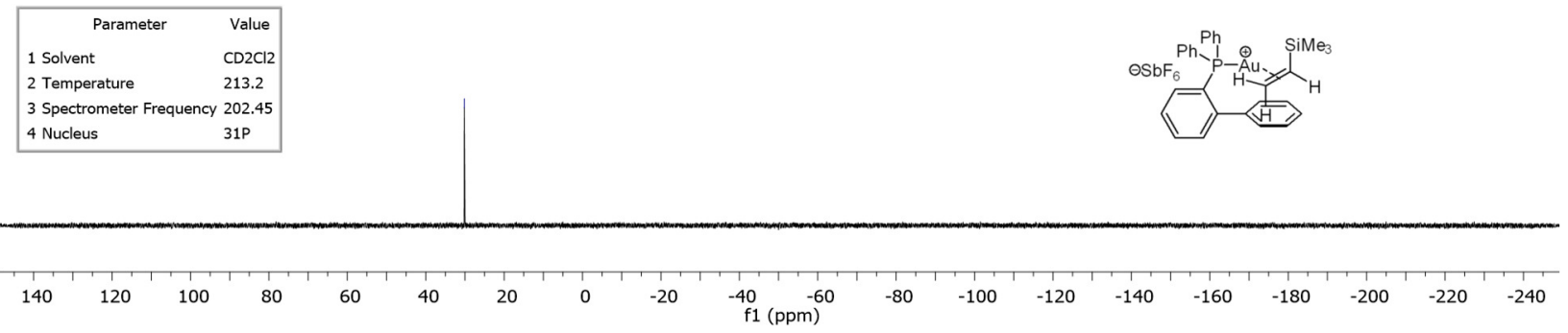

Figure S41. ${ }^{31} \mathrm{P}$ NMR spectrum of $\mathbf{5 d}\left(202.45 \mathrm{MHz}, \mathrm{CD}_{2} \mathrm{Cl}_{2},-60{ }^{\circ} \mathrm{C}\right)$. 


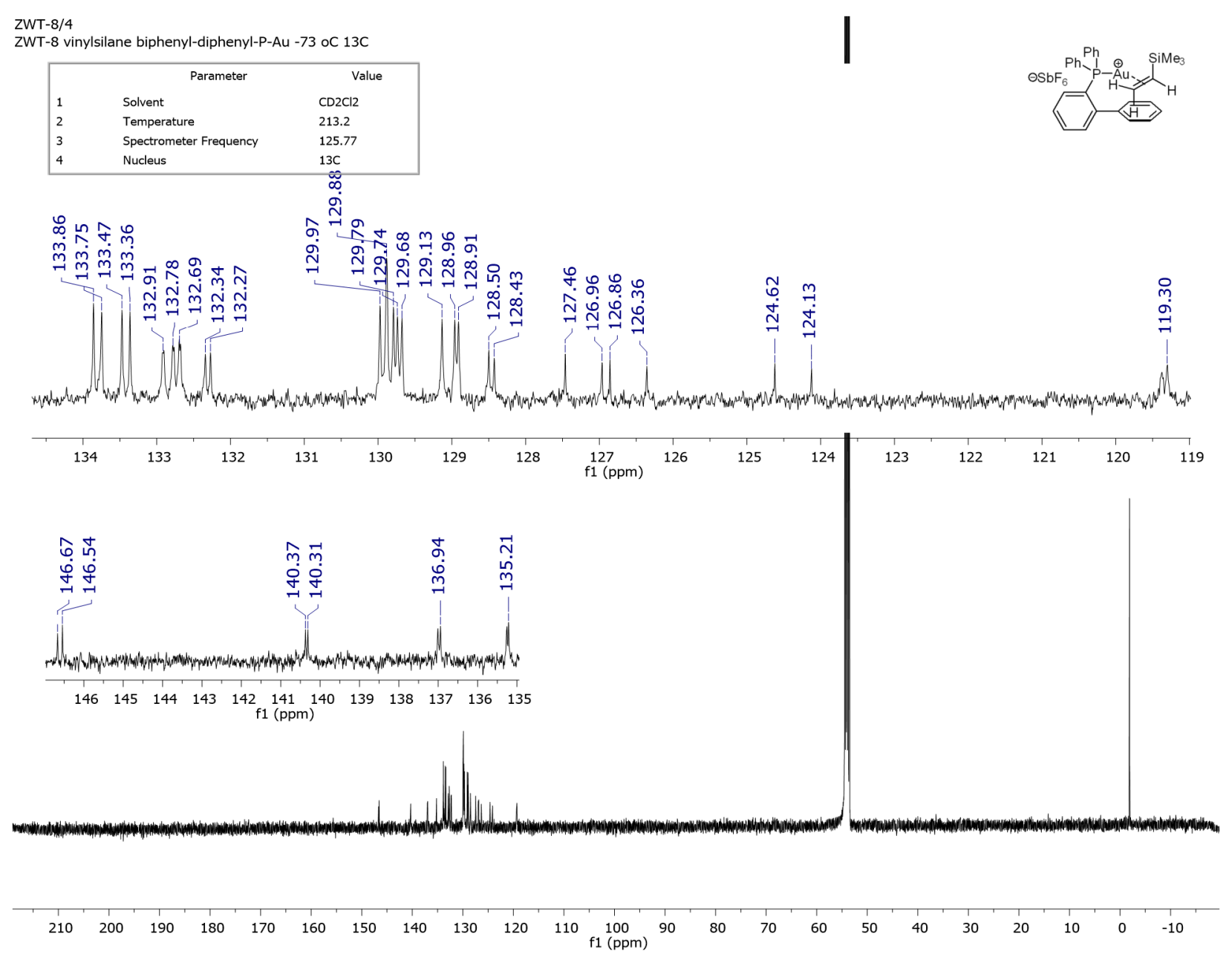

Figure S42. ${ }^{31} \mathrm{P}$ NMR spectrum of $\mathbf{5 d}\left(125.77 \mathrm{MHz}, \mathrm{CD}_{2} \mathrm{Cl}_{2},-60{ }^{\circ} \mathrm{C}\right)$. 


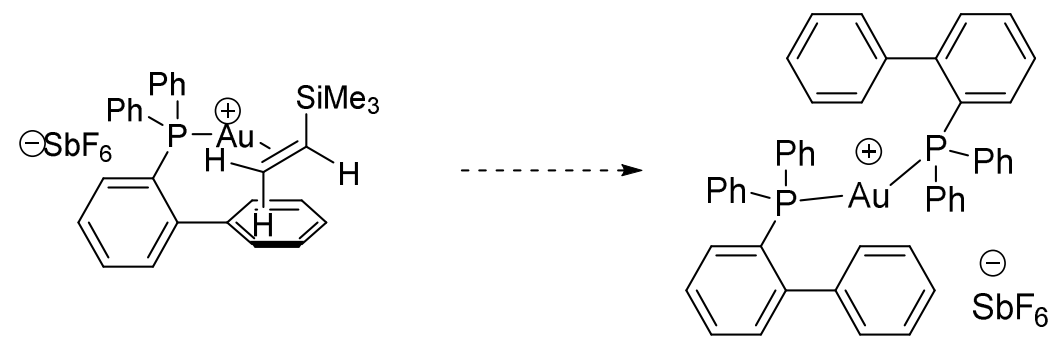

WJ-I-8 pi-complex.2.fid

wfu_P31CPD CD2Cl2 /opt/topspin1.3 zhouw12 213

$250 \mathrm{C}$

After at least 16 hours at room temperature.

ZWT-8/7

ZWT-8 vinylsilane biphenyl-diphenyl-P-Au 25oC without MeOH $31 \mathrm{P} 2$
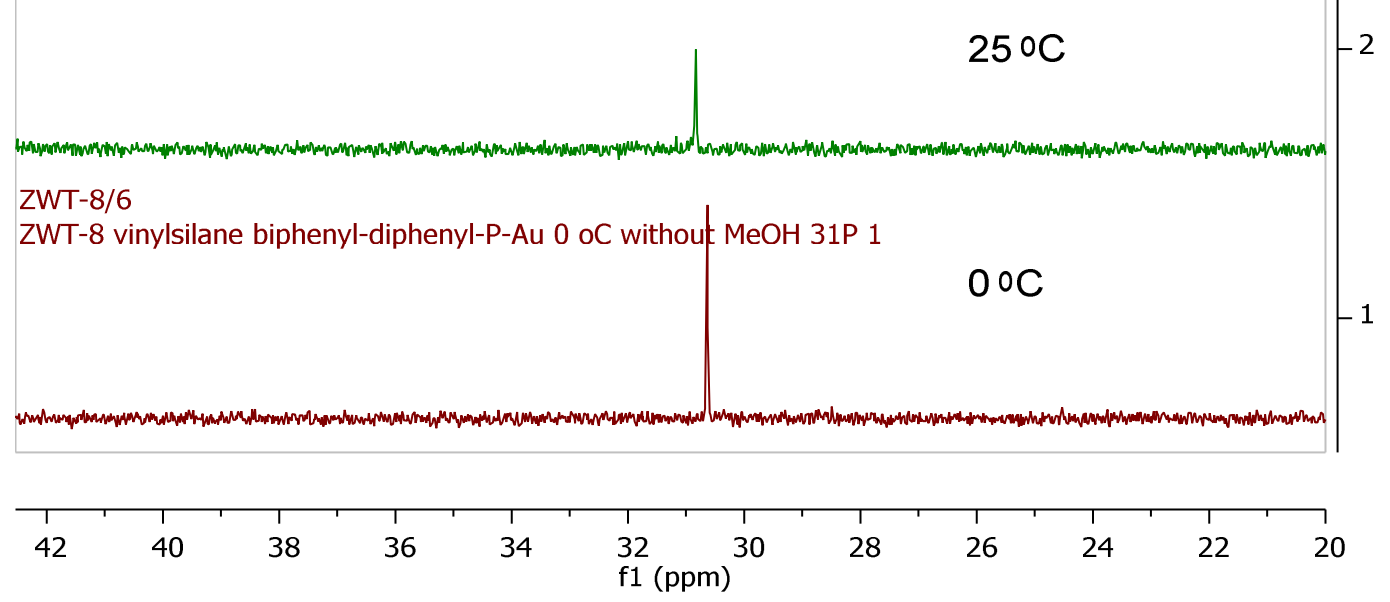

Figure S43. Decomposition of complex $\mathbf{5 d}$ in solution upon warming.
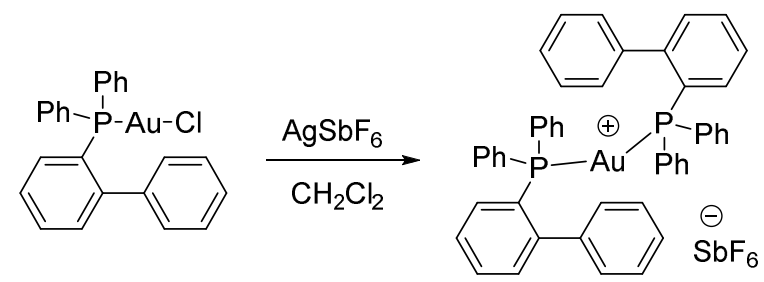

The bisphosphine decomposition product was prepared only to confirm the ${ }^{31} \mathrm{P}$ chemical shift. It was prepared by treatment of the corresponding gold chloride $(0.03 \mathrm{~g}, 0.05 \mathrm{mmol})$ with $\mathrm{AgSbF}_{6}$ $(0.018 \mathrm{~g}, 0.05 \mathrm{mmol})$ and free ligand $(0.018 \mathrm{~g}, 0.05 \mathrm{mmol})$, followed by centrifugation to remove 
the $\mathrm{AgCl}$ precipitate. The solution was decanted and concentrated in vacuo to yied a yellow oil (0.03 g, $51 \%$ yield).

${ }^{31}$ P NMR (121.5 MHz, CD2Cl2): $\delta 37.7$

Elemental Analysis for $\mathrm{C}_{96} \mathrm{H}_{76} \mathrm{Au}_{2} \mathrm{~F}_{12} \mathrm{P}_{4} \mathrm{Sb}_{2}$ calcd (found): C, 51.96 (51.70); H, 3.45 (3.71). (Corresponding yield, 51\%).

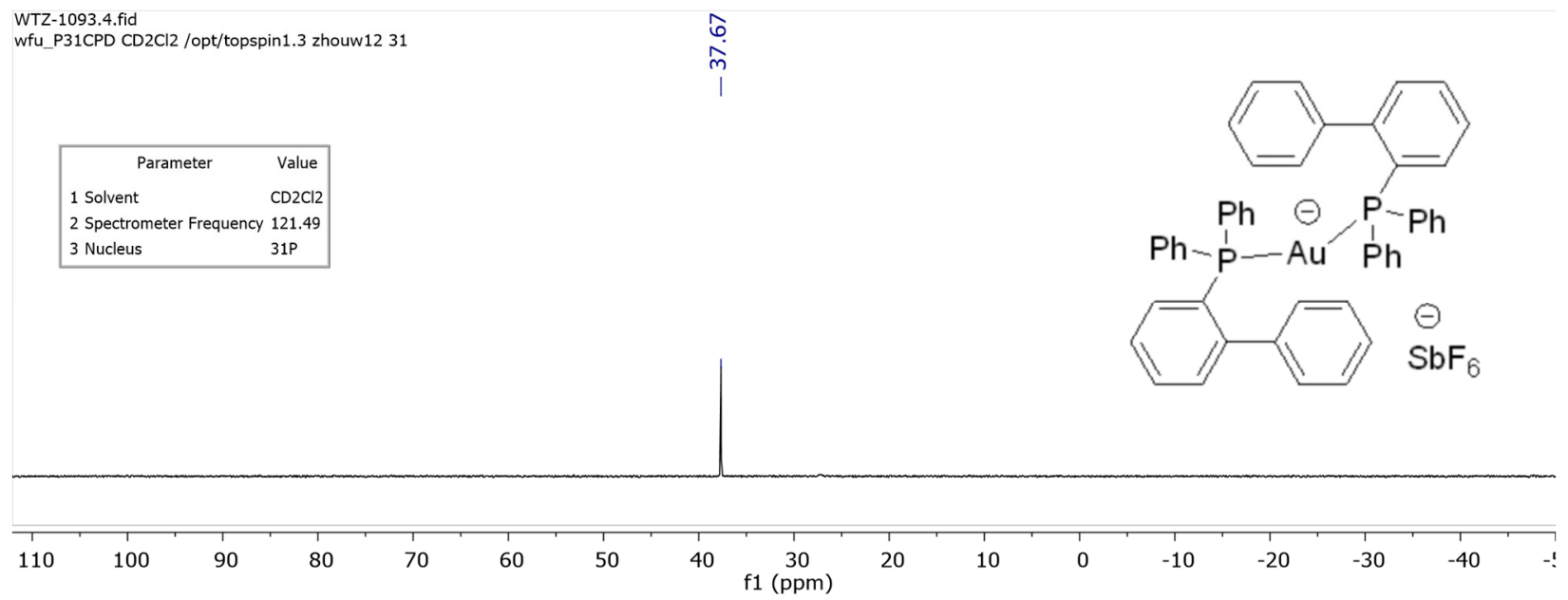

Figure S44. ${ }^{31} \mathrm{P}$ NMR Spectrum of 5 e $\left(121.5 \mathrm{MHz}, \mathrm{CD}_{2} \mathrm{Cl}_{2}\right)$. 


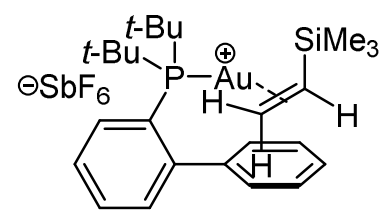

t-Bu2 $\mathrm{P}$ (o-biphenyl)]Au(Vinyltrimethylsilane)SbF6 (6d).

Gold chloride $(0.0268 \mathrm{~g}, 0.05 \mathrm{mmol})$ and AgSbF6 $(0.017,0.051 \mathrm{mmol})$ were combined in a Argon, moisture-free glove box, and to the mixture was added $0.8 \mathrm{~mL}$ of $\mathrm{CH}_{2} \mathrm{Cl}_{2}$. Vinyl trimethyl silane $(0.0295 \mathrm{~mL}, 0.2 \mathrm{mmol})$ was added and the solution was stirred for $1 \mathrm{~h}$ before filtering through a pad of celite. The solvents were removed by vacuum to yield a white solid ( $0.0392 \mathrm{~g}, 93 \%$ yield). Crystalline material was obtained by recrystallization from $\mathrm{CH} 2 \mathrm{Cl} 2 / \mathrm{Hexanes}(2: 1)$.

${ }^{1} \mathbf{H}$ NMR ( $\left.\mathbf{C D}_{2} \mathbf{C l}_{2}, \mathbf{3 0 0} \mathbf{M H z}\right)$ : d 7.89 (m, 1H), 7.62 (m, 5H), 7.24 (m, 3H), 5.54 (ddd, J=19.4, $\left.13.5 \mathrm{~Hz}, J_{\mathrm{HP}}=2.5 \mathrm{~Hz}\right), 5.2\left(\mathrm{ABXY}\right.$ Pattern, $J_{\mathrm{HaHx}}=19.4 \mathrm{~Hz}, J_{\mathrm{HbHx}}=13.5 \mathrm{~Hz}, J_{\mathrm{Hab}(\text { geminal })}=3.6 \mathrm{~Hz}$, $\left.J_{\mathrm{HP}}=2.5 \mathrm{~Hz}\right), 1.45\left(\mathrm{~d}, J_{\mathrm{HP}}=16.5 \mathrm{~Hz}, 9 \mathrm{H}\right), 1.42\left(\mathrm{~d}, J_{\mathrm{HP}}=16.5 \mathrm{~Hz}, 9 \mathrm{H}\right), 0.19(\mathrm{~s}, 9 \mathrm{H})$.

${ }^{13} \mathrm{C}$ NMR ( $\mathrm{CD}_{2} \mathrm{Cl}_{2}, 75.5 \mathrm{MHz}$, Peaks in bold are coordinated vinyl trimethyl silane): $\delta 148.67$ $(\mathrm{d}, J=13.3 \mathrm{~Hz}), 143.87$ (d, $J=7.1 \mathrm{~Hz}), 135.75$ (d, $J=8.3 \mathrm{~Hz}), 134.55$ (d, $J=3.3 \mathrm{~Hz}), 134.12$ (d, $J=7.6 \mathrm{~Hz}), 132.39$ (d, $J=2.4 \mathrm{~Hz}), 130.31,129.97,129.86,128.65,128.60(\mathrm{~d}, J=7.5 \mathrm{~Hz}), 124.5$ (d, $J=47.4 \mathrm{~Hz}), \mathbf{1 1 9 . 6 5}(\mathbf{d}, \boldsymbol{J}=\mathbf{8 . 4} \mathbf{~ H z}), 39.62(\mathrm{~d}, J=25.9 \mathrm{~Hz}), 39.31(\mathrm{~d}, J=25.9 \mathrm{~Hz}), 31.22(\mathrm{~d}, J=6.1$ $\mathrm{Hz}), \mathbf{- 1 . 1 5}$.

${ }^{31} \mathrm{P}$ NMR $\left(\mathrm{CD}_{2} \mathrm{Cl}_{2}, \mathbf{1 2 1 . 5} \mathrm{MHz}\right): \delta 68.1$.

Elemental Analysis: Cald (found) for $\mathrm{C}_{25} \mathrm{H}_{39} \mathrm{AuF}_{6} \mathrm{PSbSi}$ : C: 36.12 (36.17); H, 4.73 (4.65). (Corresponding Yield, $0.018 \mathrm{~g}, 30.2 \%$ ). 


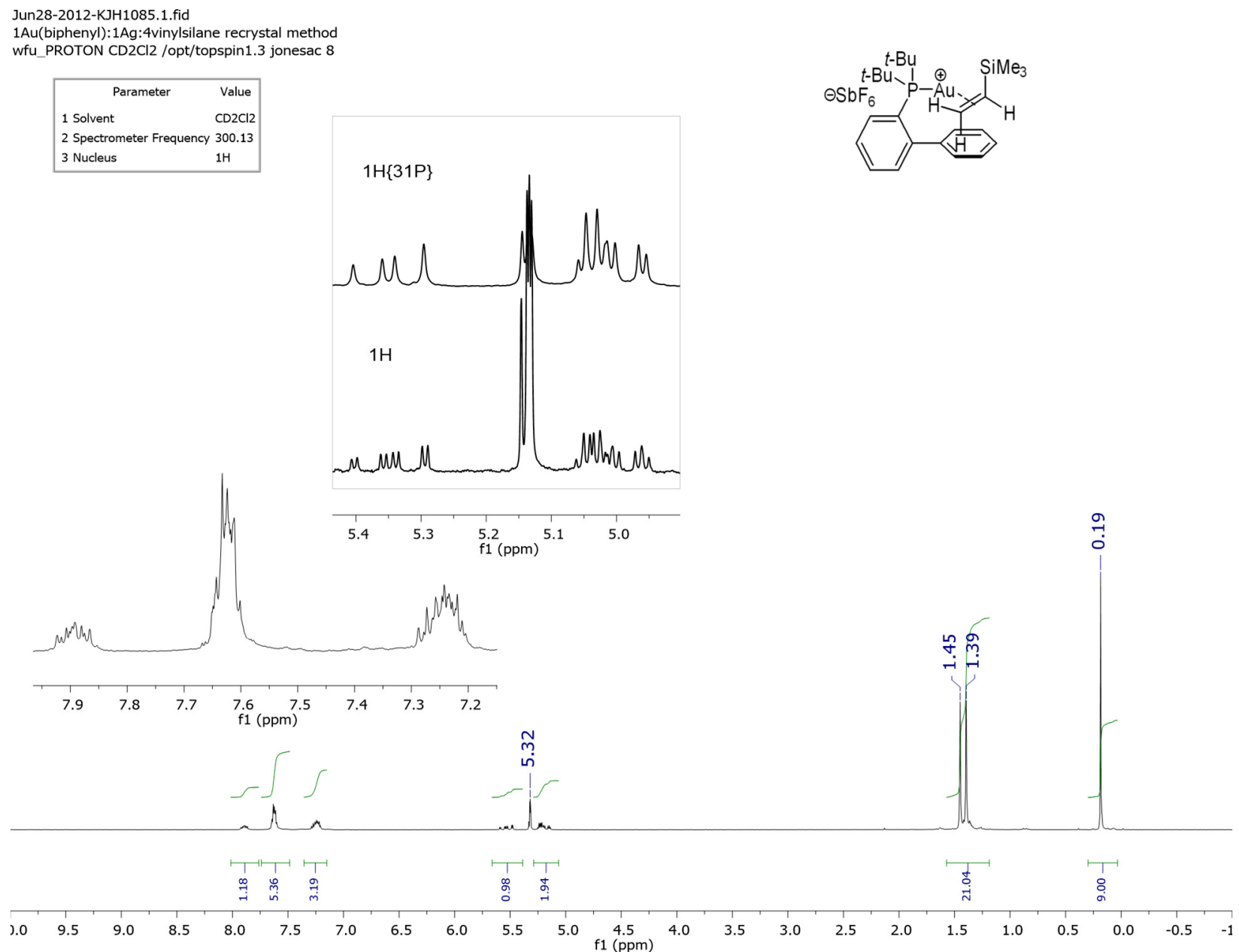

Figure S45. ${ }^{1} \mathrm{H}$ NMR for $\mathbf{5 d}\left(300.13 \mathrm{MHz}, \mathrm{CD}_{2} \mathrm{Cl}_{2}\right.$ ) (boxed inset shows 31P-decoupled spectrum).

Jun21-2012-KJH1075.2.fid

1075

wfu_P31CPD CD2Cl2/opt/topspin1.3 jonesac 22

\begin{tabular}{|ll|}
\hline \multicolumn{1}{|c|}{ Parameter } & \multicolumn{1}{c|}{ Value } \\
1 Solvent & $\mathrm{CD} 2 \mathrm{Cl} 2$ \\
2 Acquisition Date & 2012-06-21T09:25:00 \\
3 Spectrometer Frequency 121.49 \\
4 Nucleus & $31 \mathrm{P}$ \\
\hline
\end{tabular}

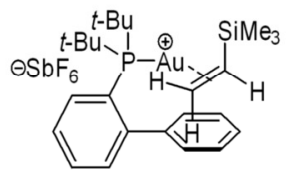

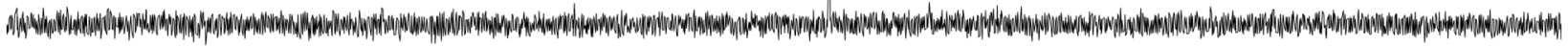

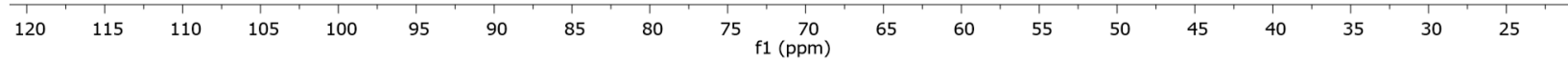

Figure S46. ${ }^{31} \mathrm{P}$ NMR Spectrum of $5 \mathbf{d}\left(121.49 \mathrm{MHz}, \mathrm{CD}_{2} \mathrm{Cl}_{2}\right)$. 
KJH1085 7-2-12.2.fid

Overnight $13 \mathrm{C}$

wfu_C13CPD_10k CD2Cl2 /opt/topspin1.3 jonesac 51
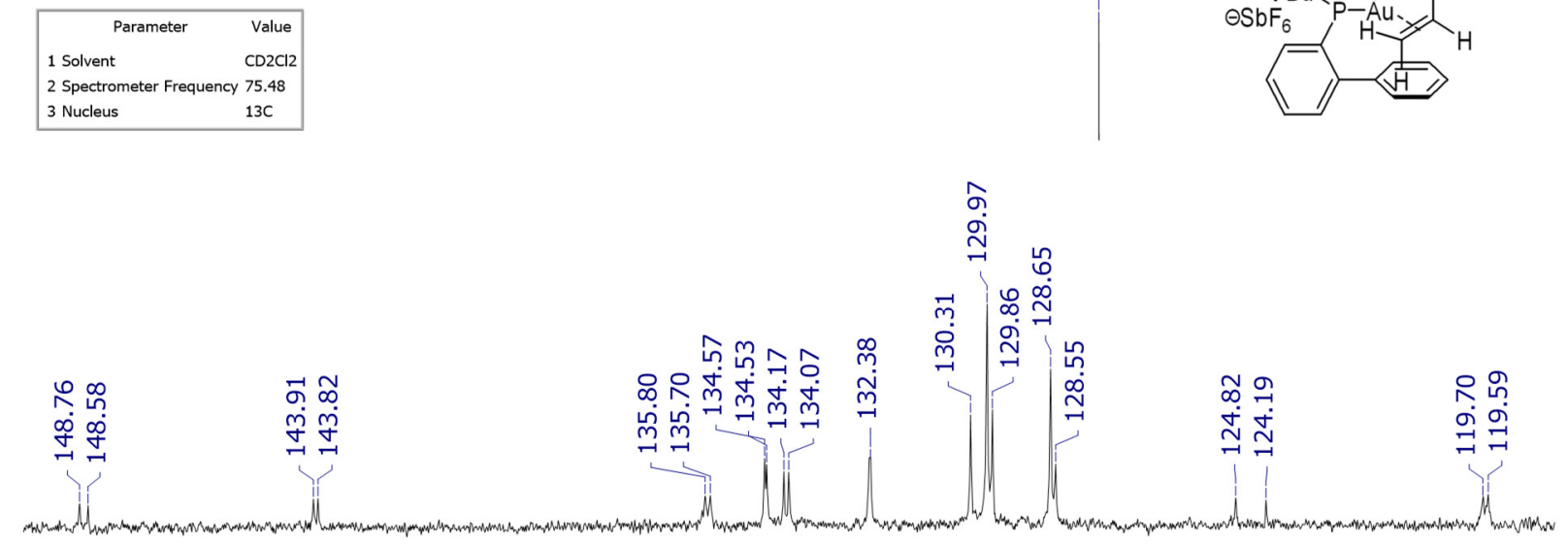

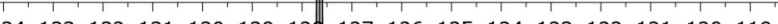
$\begin{array}{llllllll}135 & 134 & 133 & 132 & 131 & 130 & 129 & 128\end{array}$ f1 (ppm)

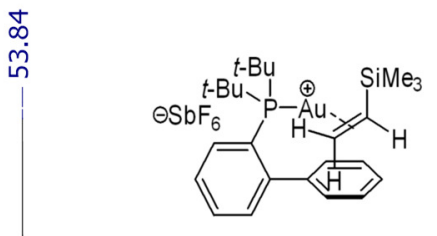

$\begin{array}{lllllllll}127 & 126 & 125 & 124 & 123 & 122 & 121 & 120 & 119\end{array}$

$\stackrel{\oplus}{\stackrel{n}{n} \infty}$

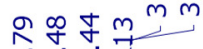

คे

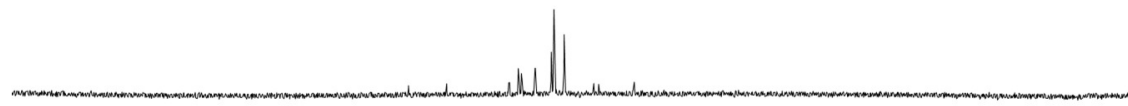

if

$\begin{array}{lllllll}190 & 190 & 180 & 170 & 160 & 150 & 140\end{array}$

Figure S47. ${ }^{13} \mathrm{C}$ NMR Spectrum of $\mathbf{5 d}\left(75.48 \mathrm{MHz}, \mathrm{CD}_{2} \mathrm{Cl}_{2}\right)$. 


\section{Alkene Exchange Study with 1c}
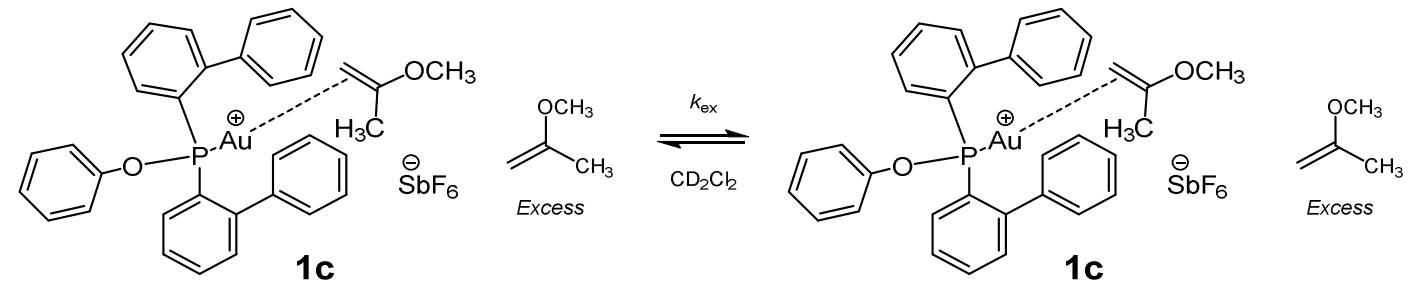

Representative Procedure for Exchange Studies: The gold methoxypropene complex (1c) was weighed into a vial $(46.7 \mathrm{mg}, 935.31 \mathrm{~g} / \mathrm{mol}, 0.05 \mathrm{mmol})$ and dissolved in $\mathrm{CD}_{2} \mathrm{Cl}_{2}(0.7 \mathrm{~mL})$ for a $0.07 \mathrm{M}$ solution. The solution was transferred to an NMR tube and NMR spectra were collected at two temperatures (calibrated by neat methanol standard). Excess methoxypropene was added by syringe in $2 \mu \mathrm{l}$ aliquots. Excess methoxypropene concentrations were determined by integrating the upfield methyl peak $(\delta 1.78)$ and comparing it to the total of the aromatic signals $(23 \mathrm{H}, \delta 7.97$ - 6.77). The natural linewidth of the $\mathrm{CH}_{3} \mathrm{O}$ signal $(\delta 3.23)$ in the initial complex was $v_{1 / 2}=2.09 \mathrm{~Hz}$. At an excess 2-methoxypropene concentration of $0.028 \mathrm{M}$, the half-height peak width for the $\mathrm{CH}_{3} \mathrm{O}$ singlet was $v_{1 / 2}=6.15 \mathrm{~Hz}$, corresponding to an excess broadening of $v_{1 / 2}$ (excess) $=4.06 \mathrm{~Hz}$. Based on this value, the rate of exchange of the enol ether with complex 1c was determined through application of the slow exchange approximation to be $k_{\mathrm{obs}}=\pi($ $v_{1 / 2}($ excess $\left.)\right)=12.75 \mathrm{~s}^{-1}$.

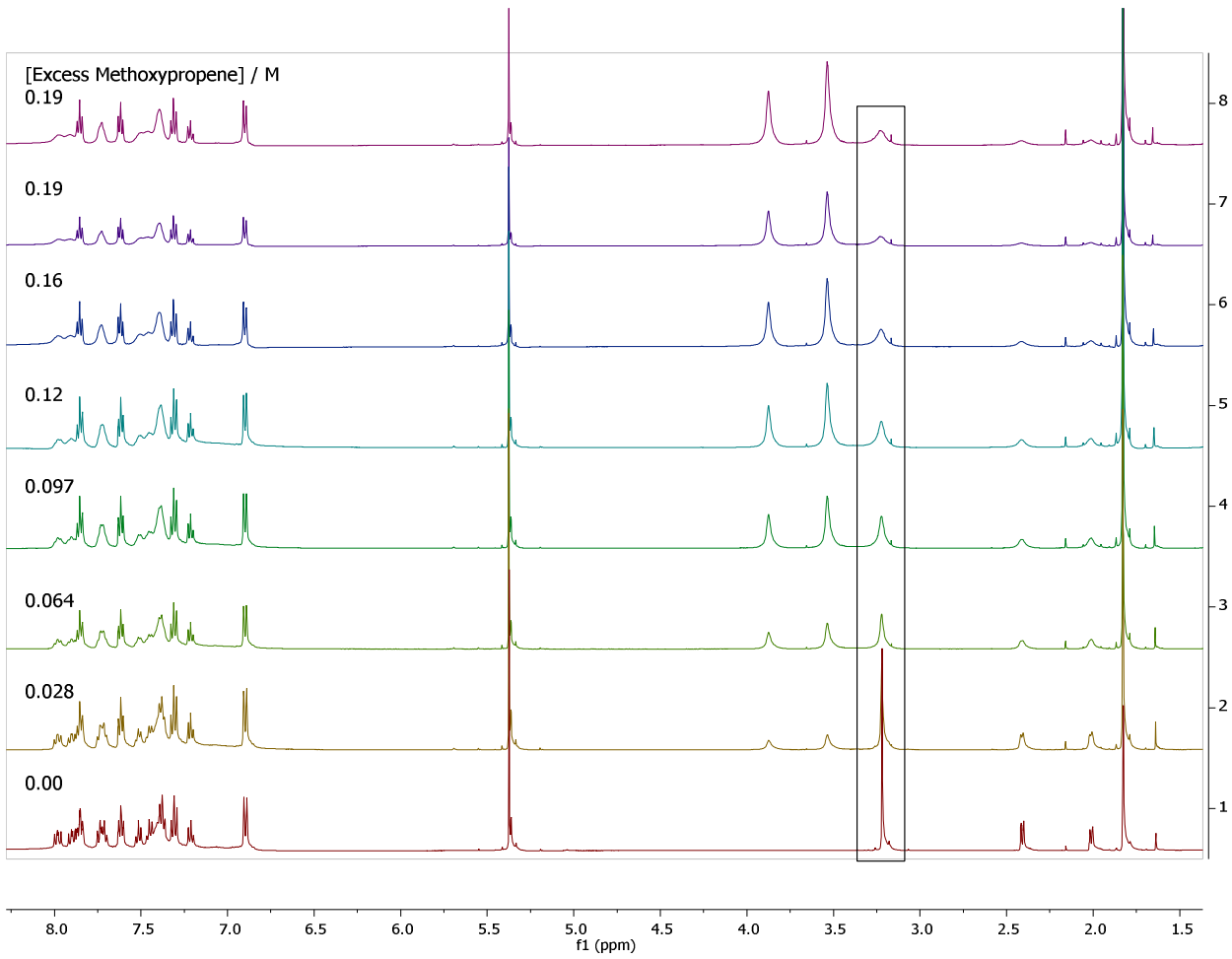

Figure S48. Representative ${ }^{1} \mathrm{H}$ NMR from $k$ exchange study of $\mathbf{1 c}$ with excess methoxypropene. 
Table S1. Data and plot of $k_{\text {obs }}$ vs. [2-methoxypropene] for the intermolecular exchange with 1c $(0.07 \mathrm{M})$ at $-2{ }^{\circ} \mathrm{C}\left(3\right.$ trials, average $\left.k_{2}=494 \mathrm{M}^{-1} \mathrm{~s}^{-1}\right)$.

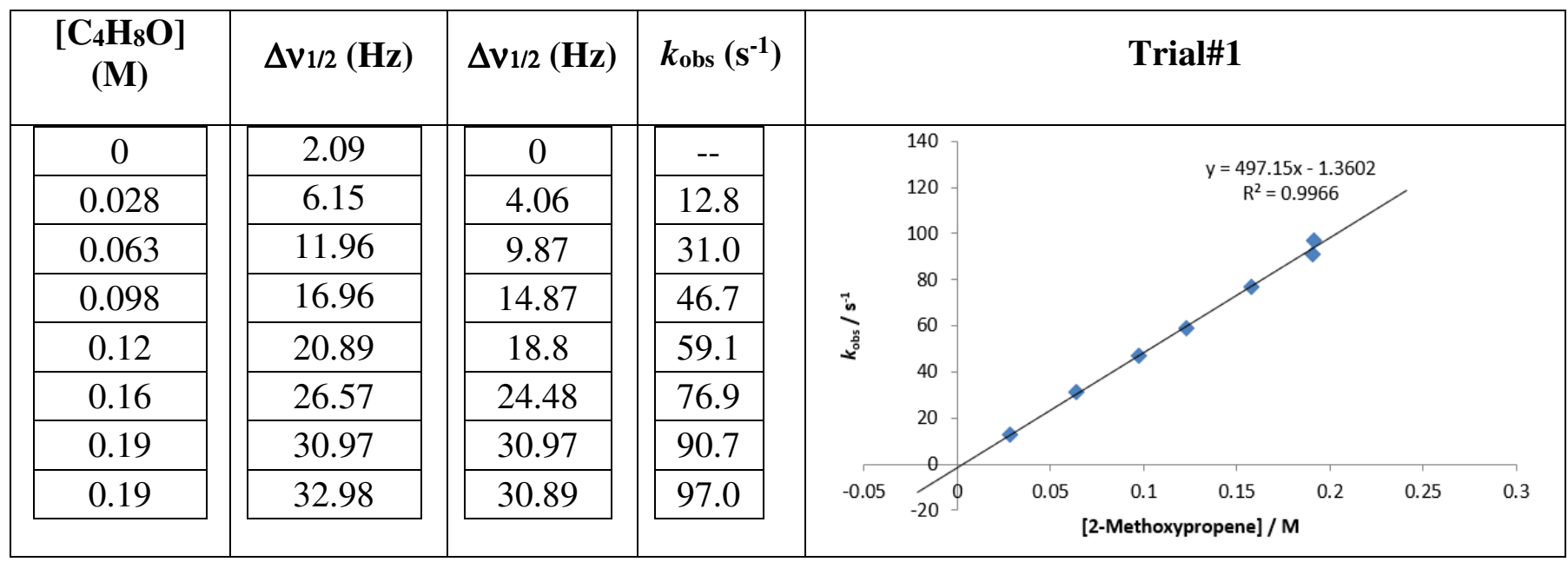

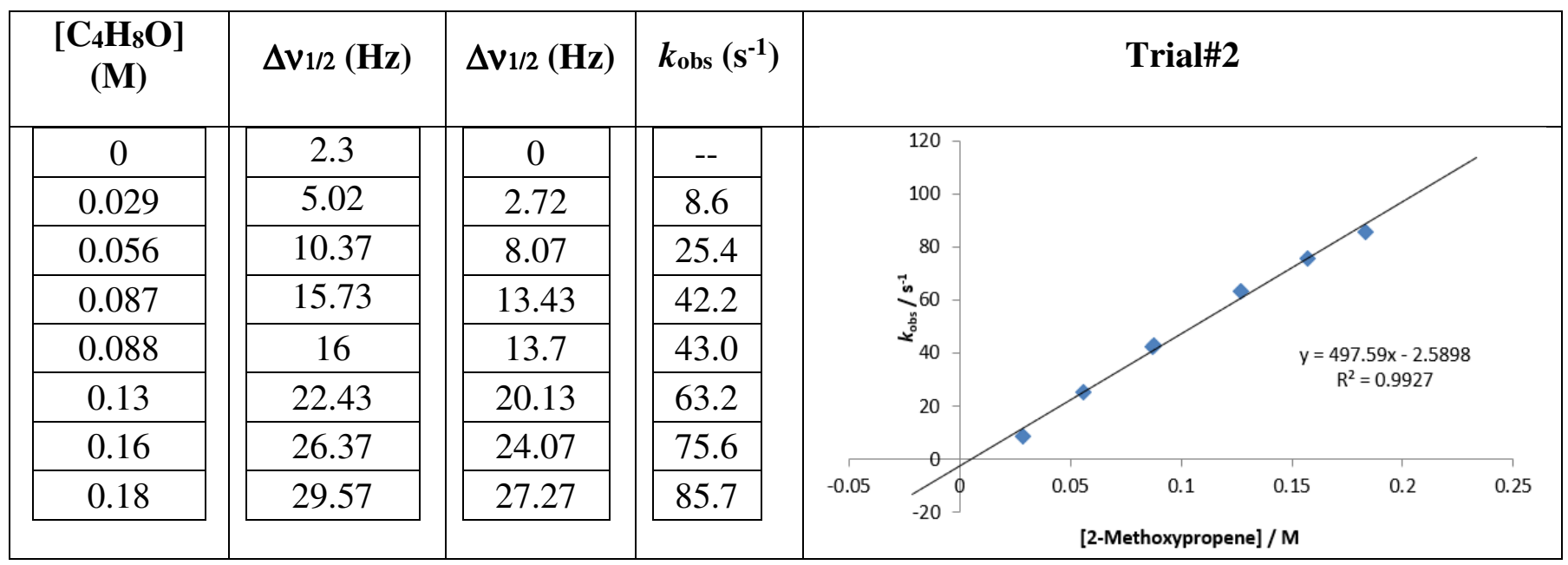

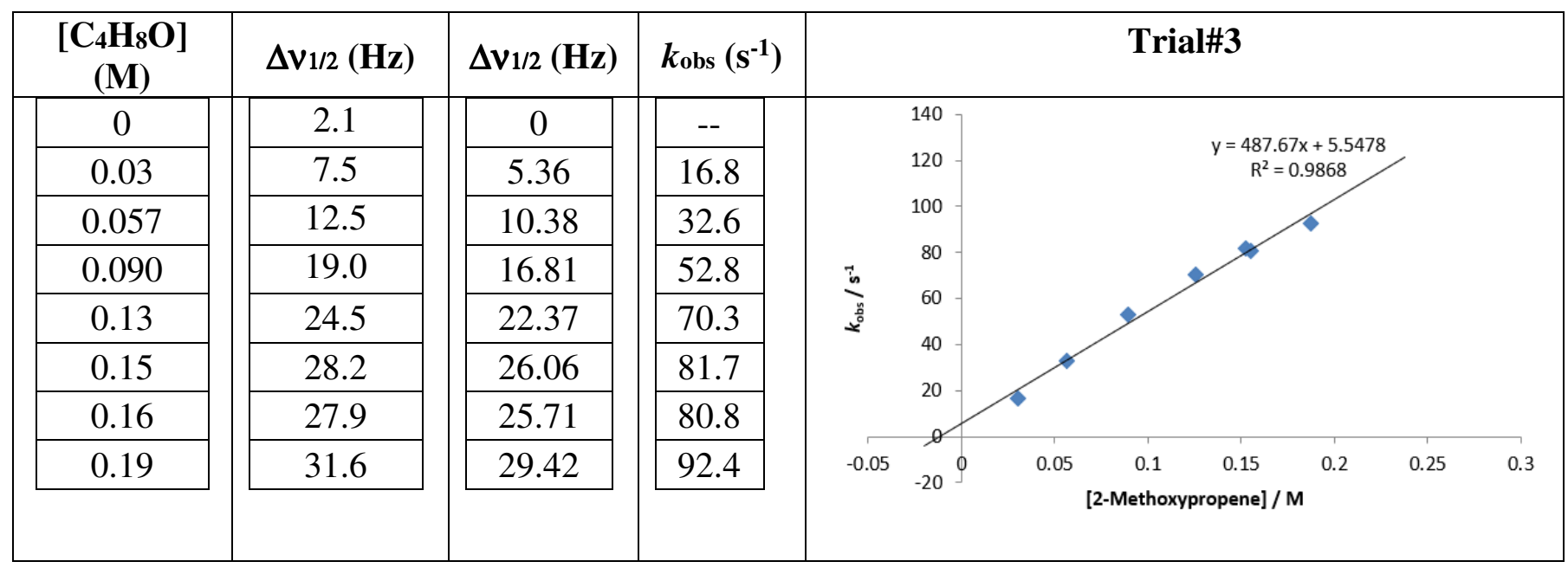


Table S2. Data and plot of $k_{\text {obs }}$ vs. [2-methoxypropene] for the intermolecular exchange with 1c $(0.07 \mathrm{M})$ at $-26^{\circ} \mathrm{C}\left(3\right.$ trials, average $\left.k_{2}=148 \mathrm{M}^{-1} \mathrm{~s}^{-1}\right)$.

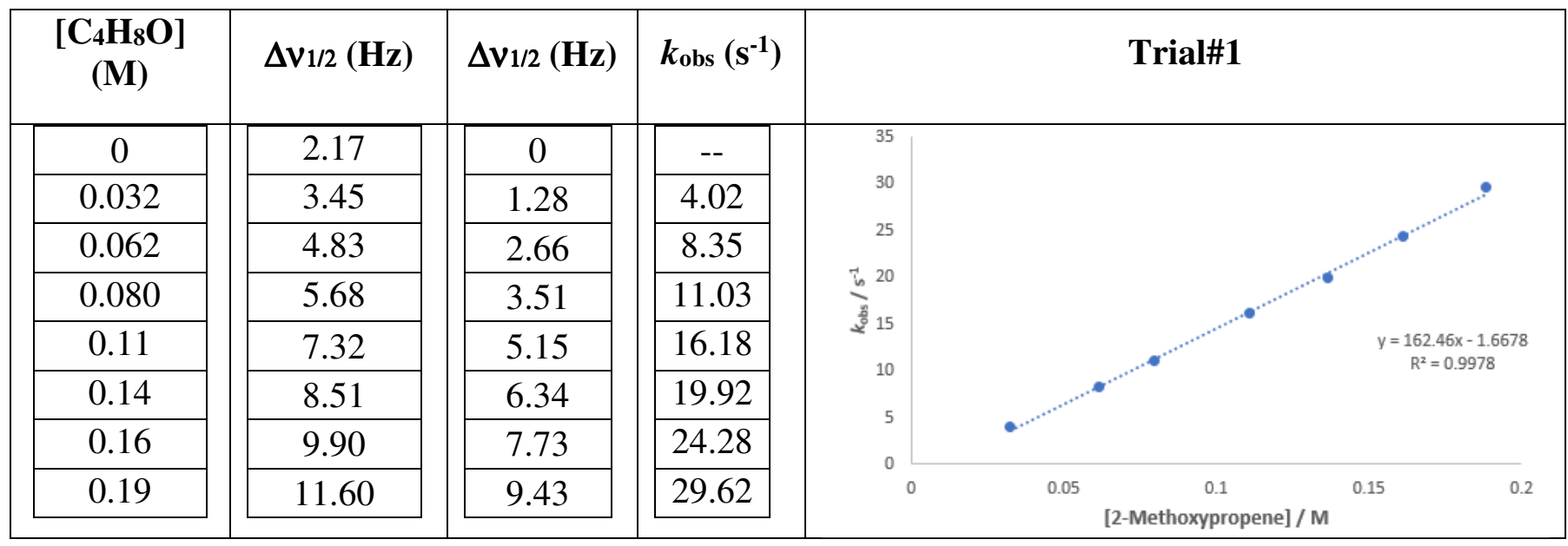

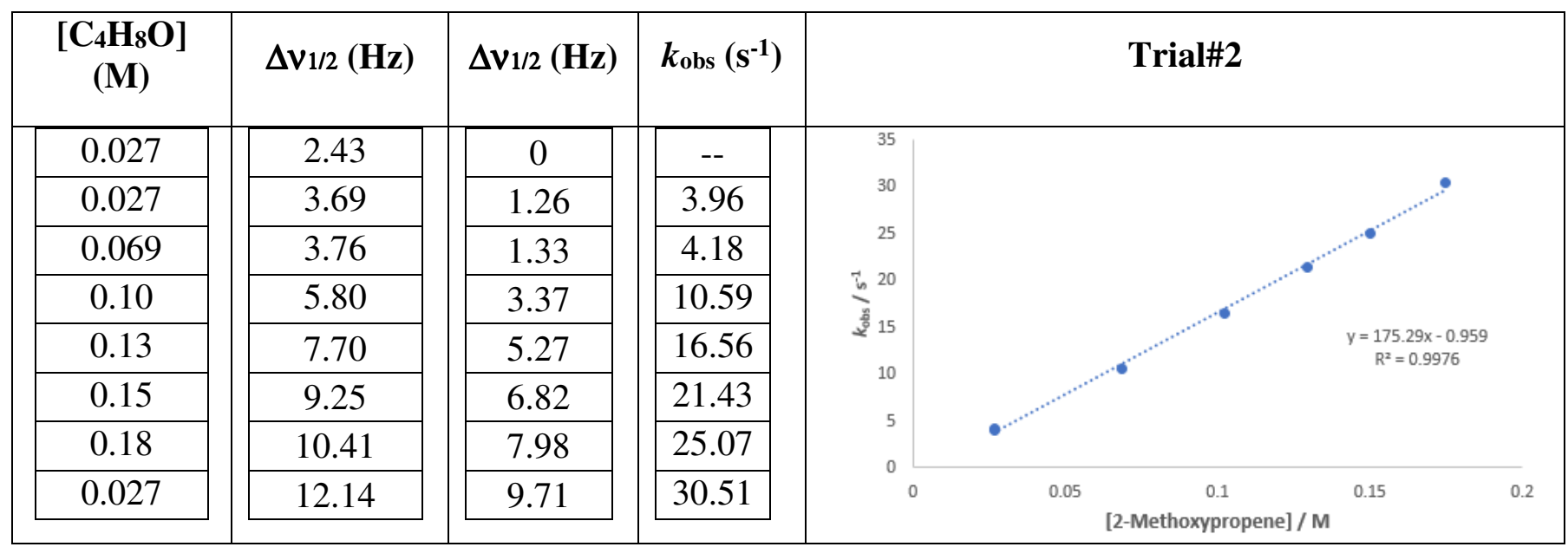

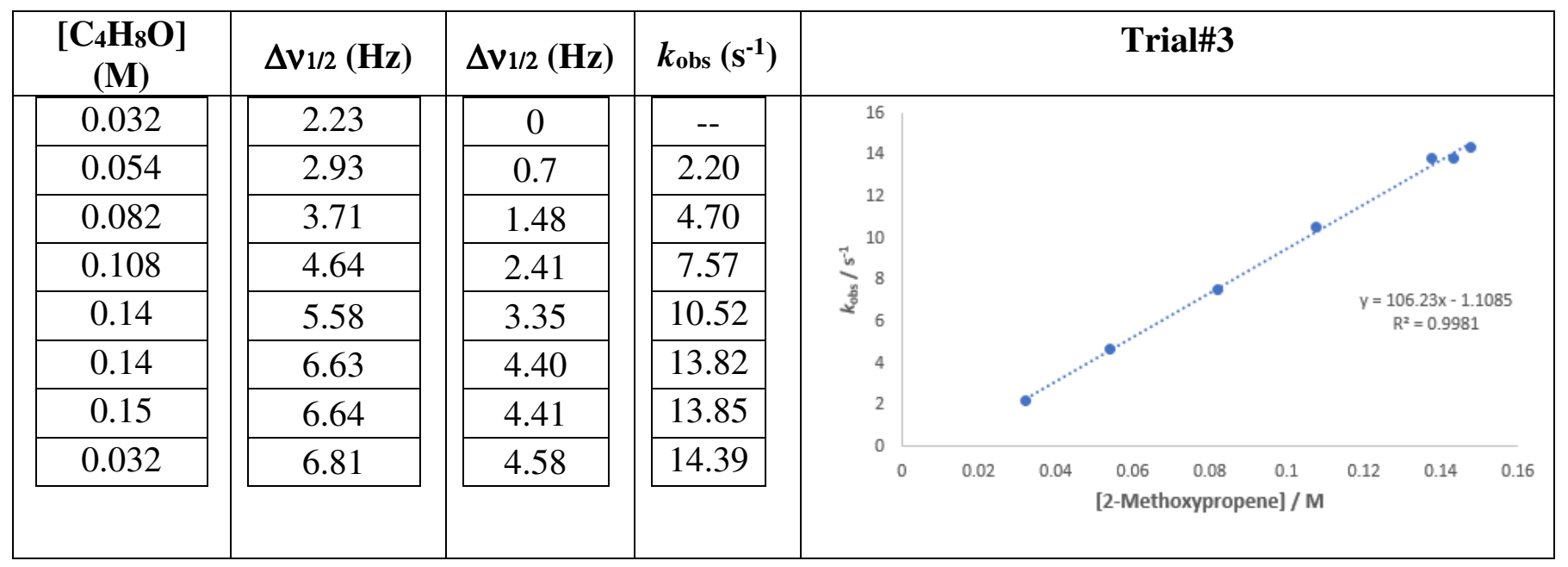




\section{VI. ${ }^{1}$ H Spectra showing Methoxypropene Hydrolysis byproducts.}

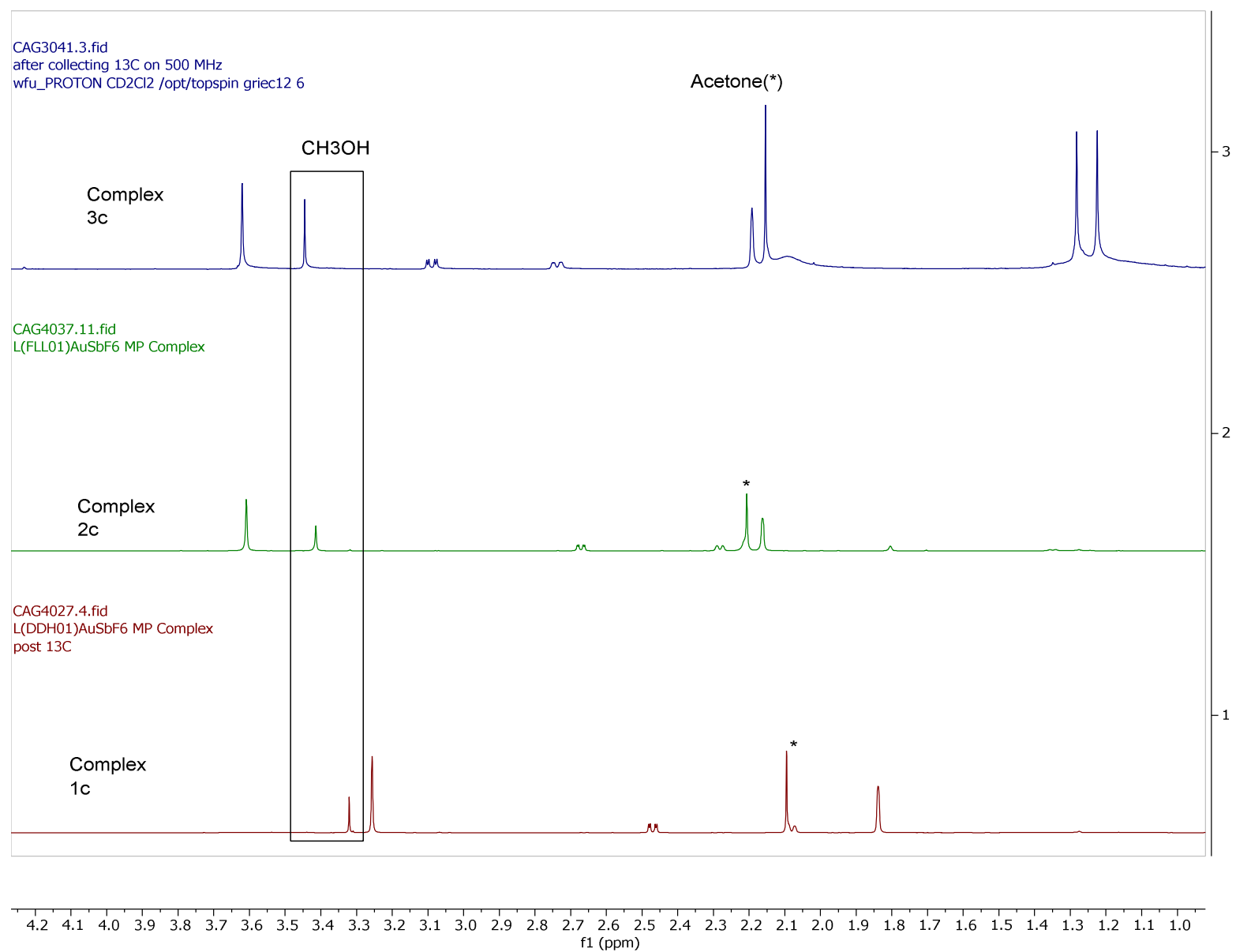

Figure S49. Insets of ${ }^{1} \mathrm{H}$ NMR spectra of methoxypropene complexes in $\mathrm{CDCl}_{2}$ after collection of ${ }^{13} \mathrm{C}$ NMR spectra, showing increase of peaks consistent with $\mathrm{MeOH}$ (expected at $3.42 \mathrm{ppm}$ ) and Acetone (expected at 2.12). ${ }^{\mathrm{S} 3}$ Slight variations in the methanol shift (3.3-3.4 ppm) and acetone shift (2.1-2.2 ppm) are presumed due to differences in media a result of the variable lewis acidity of each complex.

(S3) Fulmer, G. R.' Miller, A. J. M.; Sherden, N. H.; Gottlieb, H. E.; Nudelman, A.; Stoltz, B. M.; Bercaw, J. E.; Goldberg, K. I. Organometallics 2010, 29, 2176-2179. 